\title{
WEIGHTED BESOV AND TRIEBEL-LIZORKIN SPACES ASSOCIATED WITH OPERATORS AND APPLICATIONS
}

\author{
HUY-QUI BUI ${ }^{1}$, THE ANH BUI ${ }^{2}$ and XUAN THINH DUONG ${ }^{2}$ \\ ${ }^{1}$ Department of Mathematics, University of Canterbury, Private Bag 4800, \\ Christchurch 8140, New Zealand; \\ email: huy-qui.bui@canterbury.ac.nz,quihuybui@gmail.com \\ ${ }^{2}$ Department of Mathematics and Statistics, Macquarie University, NSW 2109, Australia; \\ email: the.bui@mq.edu.au,bt_anh80@yahoo.com,xuan.duong@mq.edu.au
}

Received 25 June 2019; accepted 5 January 2020

\begin{abstract}
Let $X$ be a space of homogeneous type and $L$ be a nonnegative self-adjoint operator on $L^{2}(X)$ satisfying Gaussian upper bounds on its heat kernels. In this paper, we develop the theory of weighted Besov spaces $\dot{B}_{p, q, w}^{\alpha, L}(X)$ and weighted Triebel-Lizorkin spaces $\dot{F}_{p, q, w}^{\alpha, L}(X)$ associated with the operator $L$ for the full range $0<p, q \leqslant \infty, \alpha \in \mathbb{R}$ and $w$ being in the Muckenhoupt weight class $A_{\infty}$. Under rather weak assumptions on $L$ as stated above, we prove that our new spaces satisfy important features such as continuous characterizations in terms of square functions, atomic decompositions and the identifications with some well-known function spaces such as Hardytype spaces and Sobolev-type spaces. One of the highlights of our result is the characterization of these spaces via noncompactly supported functional calculus. An important by-product of this characterization is the characterization via the heat kernel for the full range of indices. Moreover, with extra assumptions on the operator $L$, we prove that the new function spaces associated with $L$ coincide with the classical function spaces. Finally we apply our results to prove the boundedness of the fractional power of $L$, the spectral multiplier of $L$ in our new function spaces and the dispersive estimates of wave equations.
\end{abstract}

2010 Mathematics Subject Classification: 42B37 (primary); 47D08, 46F05, 47B38, 35K08 (secondary) 


\section{Contents}

1 Introduction

2 Preliminaries, a new class of distributions and related estimates

2.1 Muckenhoupt weights, Fefferman-Stein inequality and some estimates on spaces of homogeneous type . . . . . . . . . . 6

2.2 Kernel estimates . . . . . . . . . . . . . . . . . . 10

2.3 A new class of distributions . . . . . . . . . . . . . . . 12

2.4 Calderón reproducing formulas . . . . . . . . . . . . . . . 13

2.5 Maximal function estimates . . . . . . . . . . . . . . 17

3 Besov and Triebel-Lizorkin spaces associated with $L$ : properties and characterizations

3.1 Definitions of Besov and Triebel-Lizorkin spaces associated with $L 26$

3.2 Continuous characterizations by functions with compact supports . 29

3.3 Continuous characterizations by functions in $\mathscr{S}_{m}(\mathbb{R}) \ldots \ldots$. . . . 31

3.4 Characterizations for Triebel-Lizorkin spaces via Lusin functions and the Littlewood-Paley functions . . . . . . . . . . . . . . . 41

3.5 Weighted Triebel-Lizorkin space $\dot{F}_{\infty, q, w}^{\alpha, L}$ and its characterizations . 48

4 Atomic decompositions $\quad 59$

4.1 Atomic decompositions for Besov spaces $\dot{B}_{p, q, w}^{\alpha, L} \ldots \ldots$. . . . . 59

4.2 Atomic decompositions for Triebel-Lizorkin spaces $\dot{F}_{p, q, w}^{\alpha, L} \ldots$. . 69

5 Identifications of our new Besov and Triebel-Lizorkin spaces with known function spaces

5.1 Coincidence with $L_{w}^{p}(X)$ spaces . . . . . . . . . . . . 72

5.2 Coincidence with the weighted Hardy spaces $H_{L, w}^{p} \quad \ldots \ldots . .72$

5.3 Coincidence with the weighted $\mathrm{BMO}$ spaces $\mathrm{BMO}_{L, w}(X) \ldots \ldots 74$

5.4 Coincidence with the weighted Sobolev spaces $\dot{W}_{p, w}^{s, L} \ldots \ldots . .75$

5.5 Coincidence with weighted Hardy-Sobolev spaces $H \dot{S}_{p, w}^{s, L} \ldots \ldots$

6 Comparison with classical Besov and Triebel-Lizorkin spaces $\quad \mathbf{7 8}$

7 Applications $\quad \mathbf{8 2}$

7.1 Fractional powers . . . . . . . . . . . . 83

7.2 Spectral multiplier of Laplace transform type . . . . . . . . . . . . . 84

7.3 Dispersive estimates and Strichartz estimates . . . . . . . . . 86 


\subsubsection{Dispersive estimates and Strichartz estimates for abstract} operators. . . . . . . . . . . . 86

7.3.2 Dispersive estimates for Schrödinger operators with large rough potentials. . . . . . . . . . . . . 90

\section{References}

\section{Introduction}

Let $X$ be a space of homogeneous type, with quasidistance $d$ and $\mu$ being a nonnegative Borel measure on $X$, which satisfies the doubling property below. In this paper, we assume that $\mu(X)=\infty$.

For $x \in X$ and $r>0$, we set $B(x, r)=\{y \in X: d(x, y)<r\}$ to be the open ball with radius $r>0$ and center $x \in X$, and $V(x, r)=\mu(B(x, r))$. The doubling property of $\mu$ provides a constant $C>0$ such that

$$
V(x, 2 r) \leqslant C V(x, r)
$$

for all $x \in X$ and $r>0$.

The doubling property (1) yields a constant $n>0$ so that

$$
V(x, \lambda r) \leqslant C \lambda^{n} V(x, r),
$$

for all $\lambda \geqslant 1, x \in X$ and $r>0$, and that

$$
V(x, r) \leqslant C\left(1+\frac{d(x, y)}{r}\right)^{\tilde{n}} V(y, r),
$$

for all $x, y \in X, r>0$ and for some $\tilde{n} \in[0, n]$. See for example [24].

Let $L$ be a nonnegative self-adjoint operator on $L^{2}(X)$ which generates a semigroup $\left\{e^{-t L}\right\}_{t>0}$. Denote by $p_{t}(x, y)$ the kernel of the semigroup $e^{-t L}$. In this paper, we assume that the kernel $p_{t}(x, y)$ satisfies a Gaussian upper bound, that is, there exist two positive constants $C$ and $c$ so that for all $x, y \in X$ and $t>0$,

$$
\left|p_{t}(x, y)\right| \leqslant \frac{C}{\mu(B(x, \sqrt{t}))} \exp \left(-\frac{d(x, y)^{2}}{c t}\right) .
$$

The theory of function spaces associated with differential operators was initiated in [2] where the authors laid out the foundation for the theory of Hardy spaces associated with operators satisfying Poisson upper bounds. That work was motivated by the fact that there was a number of operators which do not fall within the scope of the Calderón-Zygmund theory of singular integrals. For example, 
consider the Schrödinger operator $L=-\Delta+V$ with $0 \leqslant V \in L_{\text {loc }}^{1}\left(\mathbb{R}^{n}\right)$ on $\mathbb{R}^{n}$ with $n \geqslant 1$. Without smoothness conditions on $V$, the associated kernel of the Riesz transform $\nabla L^{-1 / 2}$ might not satisfy the Hölder continuity or even the weaker Hörmander integral condition. As a consequence, the Riesz transform $\nabla L^{-1 / 2}$ does not belong to the class of Calderón-Zygmund operators; hence, it might not be bounded from the classical Hardy space $H^{1}\left(\mathbb{R}^{n}\right)$ to $L^{1}\left(\mathbb{R}^{n}\right)$. However, the Riesz transform $\nabla L^{-1 / 2}$ can be shown to be bounded from $H_{L}^{1}\left(\mathbb{R}^{n}\right)$ to $L^{1}\left(\mathbb{R}^{n}\right)$, where $H_{L}^{1}\left(\mathbb{R}^{n}\right)$ is the Hardy space associated with the operator $L$. See, for example, [44]. This shows the need for introducing new classes of functions. After the work [2], the study of new function spaces including BMO spaces and Hardy spaces associated with differential operators has received a great deal of attention from many researchers. See, for example, [31, 32, 44-46, 62] and the references therein.

The classical Besov and Triebel-Lizorkin spaces have played an essential role in approximation theory and partial differential equations and have been developed extensively on Euclidean spaces $\mathbb{R}^{n}$. (See $[4-6,10-13,34,35,55,60$, 61, 63].) Note that the classical Triebel-Lizorkin spaces can be considered as generalizations of other classical spaces such as Lebesgue spaces, BMO (bounded mean oscillation) spaces, Hardy spaces and Sobolev spaces. Motivated by the above research direction, recently the theory of new Besov and Triebel-Lizorkin spaces associated with differential operators $L$ has been developed by many mathematicians in various settings. See, for example, $[9,16,17,26,28,50-$ $52,54,64]$ and the references therein. We now list some bodies of work related to this new research development.

(i) Using the existence of the approximation of identity, the authors developed the theory of Besov spaces $\dot{B}_{p, q}^{s}$ and Triebel-Lizorkin spaces $\dot{F}_{p, q}^{s}$ for a range $1 \leqslant p, q \leqslant \infty$ and $s \in(-\theta, \theta)$ for some $\theta \in(0,1)$ on metric measure spaces with polynomial volume growths [39] and on spaces with doubling and reverse doubling measures [42].

(ii) In [54], the authors introduced new Besov and Triebel-Lizorkin spaces associated with the Hermite operator with a full range of indices. Similarly to the classical results, they proved the frame decompositions for these spaces by making use of estimates of eigenvectors of the Hermite operators. Similar results was also proved for the Laguerre operator in [51]. The theory of these function spaces was further developed in $[16,17]$ where the authors proved molecular and atomic decomposition theorems and square function characterizations for these spaces.

(iii) In [9], the authors introduced the theory of Besov spaces $\dot{B}_{p, q}^{s, L}$ associated with an operator $L$ satisfying Poisson estimates on metric spaces with a measure 
enjoying a polynomial upper bound on volume growth. However, the indices are only for $1 \leqslant p, q \leqslant \infty$ and $-1<s<1$. The restriction of indices is due to some technical reasons and the absence of a suitable space of distributions.

(iv) Recently, under the assumption that $L$ is a nonnegative self-adjoint operator satisfying Gaussian upper bounds, Hölder continuity and Markov semigroup properties, the frame decompositions of Besov and Triebel-Lizorkin spaces associated with $L$ with full range of indices were studied in [26, 36, 50]. This theory has a wide range of applications from the setting of Lie groups to Riemannian manifolds.

Our main aim in this paper is to conduct the theory of weighted Besov and Triebel-Lizorkin spaces associated with a nonnegative self-adjoint operator. In contrast to [36, 50], we assume the Gaussian upper bound (GE) but do not assume Hölder continuity on the heat kernels or the Markov properties (the conservation property). This allows our theory to cover a wider range of applications including regularity estimates for certain singular integrals with rough kernels which are beyond the class of Calderón-Zygmund operators. In this article, in order to introduce the weighted Besov and Triebel-Lizorkin spaces, we adapt ideas in $[55,60,61]$ to make use of spectral decompositions of nonnegative self-adjoint operators. Our point of view and approach are motivated and influenced by a large body of works in the classical setting, especially [8, 11, 12, 22, 35, 55, 58]. Our development on the new theory of weighted Besov and Triebel-Lizorkin spaces can be viewed as a generalization of the classical settings in $\mathbb{R}^{n}$. We would like to point out that the extension of those relevant classical techniques and tools to the current setting is highly nontrivial including new ideas concerning Calderón reproducing formulas in our new setting with respect to new space of distributions and a number of estimates related to maximal functions associated with functional calculus of $L$. These are of interest in their own right and should be useful in future research in the field. For convenience we list the main contributions of the article:

- Section 2 recalls the Fefferman-Stein maximal inequality and gives the definition of a new class of distributions. More importantly, we prove a number of Calderón reproducing formulas and some maximal function estimates which play a crucial role in the proofs of the main results.

- Section 3 gives definitions of the weighted Besov and Triebel-Lizorkin spaces. Then we prove continuous characterizations including square function characterizations via heat kernels of the new weighted Besov and TriebelLizorkin spaces. A significant advance of our paper is in Theorem 3.6, where we prove the characterization of the new spaces via noncompactly supported spectral functional calculus. In particular, this theorem essentially solves in the current setting a natural open problem raised in [9, Remark, pages 2480-2481], for an 
important class of 'kernel functions' and for the full range of indices. Moreover, we also study the Triebel-Lizorkin space $\dot{F}_{p, q, w}^{\alpha, L}$ with $p=\infty$, which does not seem to be considered in this setting in the literature before.

- Section 4 establishes atomic decompositions of these new spaces where we prove similar results to the classical function spaces in the Euclidean setting.

- Section 5 derives identifications of these new spaces with well-known spaces such as Hardy spaces and Sobolev-Hardy spaces associated with operators.

- Section 6 compares our new spaces with classical Besov and Triebel-Lizorkin spaces.

- Section 7 proves the boundedness of singular integrals including fractional power of $L$ and spectral multipliers $m(L)$ on the new function spaces and some dispersive estimates for wave equations. For some particular operators $L$, our results recover a number of estimates in $[48,49]$.

We note that, after a preliminary version of the manuscript was completed, we learned that the atomic decompositions have been obtained independently in [37] by using a different approach. It is worth noticing that in comparison with [37], our assumptions on the operator $L$ and the underlying spaces $X$ are weaker than those in [37]. In our paper, $L$ is assumed to be nonnegative, selfadjoint and has a Gaussian upper bound, whereas apart from these conditions, in [37], the operator $L$ is assumed to satisfy some additional conditions such as Hölder continuity estimate and Markov semigroup property. Moreover, the reverse doubling condition and noncollapsing condition for the underlying space are additionally required in [37]. It is worth emphasizing that our approach can be adapted to study the generalized Besov and Triebel-Lizorkin spaces as in [66] or Besov and Triebel-Lizorkin spaces with variable exponents which are associated with operators satisfying the Gaussian upper bounds of order $m$. We believe that the ideas and techniques developed in the current work will stimulate further research in harmonic analysis of function spaces associated with operators. Throughout the paper, we usually use $C$ and $c$ to denote positive constants that are independent of the main parameters involved but whose values may differ from line to line. We will write $A \lesssim B$ if there is a universal constant $C$ so that $A \leqslant C B$ and $A \sim B$ if $A \lesssim B$ and $B \lesssim A$.

\section{Preliminaries, a new class of distributions and related estimates}

\subsection{Muckenhoupt weights, Fefferman-Stein inequality and some estimates} on spaces of homogeneous type. To simplify notation, we will often use $B$ for $B\left(x_{B}, r_{B}\right)$. Also given $\lambda>0$, we will write $\lambda B$ for the $\lambda$-dilated ball, which is the ball with the same center as $B$ and with radius $r_{\lambda B}=\lambda r_{B}$. For each ball $B \subset X$, 
we set

$$
S_{0}(B)=4 B \quad \text { and } \quad S_{j}(B)=2^{j} B \backslash 2^{j-1} B \text { for } j \geqslant 3 \text {. }
$$

For $x, y \in X$ and $r>0$, we denote $V(x \wedge y, r)=\min \{V(x, r), V(y, r)\}$ and $V(x \vee y, r)=\max \{V(x, r), V(y, r)\}$.

A weight $w$ is a nonnegative measurable and locally integrable function on $X$. Let $w$ be a weight. For any measurable set $E \subset X$, we denote $w(E):=$ $\int_{E} w(x) d \mu(x)$ and $V(E)=\mu(E)$. We denote

$$
f_{E} h(x) d \mu(x)=\frac{1}{V(E)} \int_{E} h(x) d \mu(x) .
$$

For $1 \leqslant p \leqslant \infty$, let $p^{\prime}$ be the conjugate exponent of $p$, that is, $1 / p+1 / p^{\prime}=1$.

We say that a weight $w \in A_{p}, 1<p<\infty$, if the following holds true:

$$
[w]_{A_{p}}:=\sup _{B: \text { balls }}\left(f_{B} w(x) d \mu(x)\right)^{1 / p}\left(f_{B} w(x)^{-1 /(p-1)} d \mu(x)\right)^{(p-1) / p}<\infty \text {. }
$$

For $p=1$, we say that $w \in A_{1}$ if there is a constant $C$ such that for every ball $B \subset X$,

$$
f_{B} w(y) d \mu(y) \leqslant C w(x) \text { for a.e. } x \in B \text {. }
$$

We set $A_{\infty}=\bigcup_{p \geqslant 1} A_{p}$.

The reverse Hölder classes are defined in the following way: a weight $w \in$ $R H_{q}, 1<q<\infty$, if there is a constant $C$ such that for any ball $B \subset X$,

$$
\left(f_{B} w(y)^{q} d \mu(y)\right)^{1 / q} \leqslant C f_{B} w d \mu(x) .
$$

The endpoint $q=\infty$ is given by the condition: $w \in R H_{\infty}$, if there is a constant $C$ such that for any ball $B \subset X$,

$$
w(x) \leqslant C f_{B} w(y) d \mu(y) \text { for a.e. } x \in B .
$$

For $w \in A_{\infty}$ and $0<p<\infty$, the weighted space $L_{w}^{p}(X)$ is defined by

$$
\left\{f: \int_{X}|f(x)|^{p} w(x) d \mu(x)<\infty\right\}
$$

with the norm

$$
\|f\|_{p, w}=\left(\int_{X}|f(x)|^{p} w(x) d \mu(x)\right)^{1 / p} .
$$

We sum up some of the standard properties of classes of weights in [58] in the following lemma. 
LEMMA 2.1. The following properties hold:

(i) $A_{1} \subset A_{p} \subset A_{q}$ for $1<p \leqslant q<\infty$.

(ii) If $w \in A_{p}, 1<p<\infty$, then there exists $1<r<p<\infty$ such that $w \in A_{r}$.

(iii) If $w \in A_{p}, 1<p<\infty$, then $w^{1-p^{\prime}} \in A_{p^{\prime}}$.

(iv) If $w \in A_{p}, 1 \leqslant p<\infty$, then there exists $C>0$ so that for any ball $B$ and any measurable subset $E \subset B$, we have

$$
\frac{w(B)}{w(E)} \leqslant C\left(\frac{V(B)}{V(E)}\right)^{p} .
$$

(v) If $w \in R H_{p}, 1<p<\infty$, then there exists $1<p<q<\infty$ such that $w \in R H_{q}$.

(vi) $\bigcup_{1<p<\infty} A_{p} \subset \bigcup_{1<q<\infty} R H_{q}$.

For $w \in A_{\infty}$, we define $q_{w}=\inf \left\{q: w \in A_{q}\right\}$ and $r_{w}=\sup \left\{r: w \in R H_{r}\right\}$.

Let $w \in A_{\infty}$ and $0<r<\infty$. The weighted Hardy-Littlewood maximal function $\mathcal{M}_{r, w}$ is defined by

$$
\mathcal{M}_{r, w} f(x)=\sup _{x \in B}\left(\frac{1}{w(B)} \int_{B}|f(y)|^{r} w(y) d \mu(y)\right)^{1 / r},
$$

where the sup is taken over all balls $B$ containing $x$. We will drop the subscripts $r$ or $w$ when either $r=1$ or $w \equiv 1$.

Let $w \in A_{\infty}$ and $0<r<\infty$. It is well known that

$$
\left\|\mathcal{M}_{r, w} f\right\|_{p, w} \lesssim\|f\|_{p, w}
$$

for all $p>r$.

Moreover, let $0<r<\infty$ and $p>r$. Then we have

$$
\left\|\mathcal{M}_{r} f\right\|_{p, w} \lesssim\|f\|_{p, w}
$$

for $w \in A_{p / r}$.

The following elementary estimates will be used frequently; see, for example, [21].

LEMMA 2.2. Let $\epsilon>0$. 
(a) For any $p \in[1, \infty]$, we have

$$
\left(\int_{X}\left[\left(1+\frac{d(x, y)}{s}\right)^{-n-\epsilon}\right]^{p} d \mu(y)\right)^{1 / p} \lesssim V(x, s)^{1 / p}
$$

for all $x \in X$ and $s>0$.

(b) For any $f \in L_{\text {loc }}^{1}(X)$, we have

$$
\int_{X} \frac{1}{V(x \wedge y, s)}\left(1+\frac{d(x, y)}{s}\right)^{-n-\epsilon}|f(y)| d \mu(y) \lesssim \mathcal{M} f(x),
$$

for all $x \in X$ and $s>0$.

We recall the Fefferman-Stein vector-valued maximal inequality and its variant in [38, 50]. For $0<p<\infty, 0<q \leqslant \infty, 0<r<\min \{p, q\}$ and $w \in A_{p / r}$, we then have, for any sequence of measurable functions $\left\{f_{v}\right\}$,

$$
\left\|\left(\sum_{v}\left|\mathcal{M}_{r} f_{v}\right|^{q}\right)^{1 / q}\right\|_{p, w} \lesssim\left\|\left(\sum_{v}\left|f_{v}\right|^{q}\right)^{1 / q}\right\|_{p, w} .
$$

For any measurable function $F: X \times \mathbb{R}_{+} \rightarrow \mathbb{C}$ with respect to the product measure $d \mu \times d u$, one has, for $0<p<\infty, 0<q \leqslant \infty, w \in A_{p / r}$ and $0<r<\min \{p, q\}$,

$$
\left\|\left(\int_{0}^{\infty}\left[\mathcal{M}_{r}(F(\cdot, u))(\cdot)\right]^{q} \frac{d u}{u}\right)^{1 / q}\right\|_{p, w} \lesssim\left\|\left(\int_{0}^{\infty}|(F(\cdot, u))(\cdot)|^{q} \frac{d u}{u}\right)^{1 / q}\right\|_{p, w} .
$$

The Young's inequality and (8) imply the following inequality: if $\left\{a_{v}\right\} \in \ell^{q} \cap \ell^{1}$, then

$$
\left\|\sum_{j}\left(\sum_{v}\left|a_{j-v} \mathcal{M}_{r} f_{v}\right|^{q}\right)^{1 / q}\right\|_{p, w} \lesssim\left\|\left(\sum_{v}\left|f_{v}\right|^{q}\right)^{1 / q}\right\|_{p, w} .
$$

We will now recall an important covering lemma in [23].

LEMMA 2.3. There exists a collection of open sets $\left\{Q_{\tau}^{k} \subset X: k \in \mathbb{Z}, \tau \in I_{k}\right\}$, where $I_{k}$ denotes certain (possibly finite) index set depending on $k$ and constants $\rho \in(0,1), a_{0} \in(0,1]$ and $\kappa_{0} \in(0, \infty)$ such that

(i) $\mu\left(X \backslash \bigcup_{\tau} Q_{\tau}^{k}\right)=0$ for all $k \in \mathbb{Z}$;

(ii) if $i \geqslant k$, then either $Q_{\tau}^{i} \subset Q_{\beta}^{k}$ or $Q_{\tau}^{i} \cap Q_{\beta}^{k}=\emptyset$;

(iii) for every $(k, \tau)$ and each $i<k$, there exists a unique $\tau^{\prime}$ such $Q_{\tau}^{k} \subset Q_{\tau^{\prime}}^{i}$; 
(iv) the diameter $\operatorname{diam}\left(Q_{\tau}^{k}\right) \leqslant \kappa_{0} \rho^{k}$;

(v) each $Q_{\tau}^{k}$ contains certain ball $B\left(x_{Q_{\tau}^{k}}, a_{0} \rho^{k}\right)$.

REMARK 2.4. Since the constants $\rho$ and $a_{0}$ are not essential in the paper, without loss of generality, we may assume that $\rho=a_{0}=1 / 2$. We then fix a collection of open sets in Lemma 2.3 and denote this collection by $\mathcal{D}$. We call open sets in $\mathcal{D}$ the dyadic cubes in $X$ and $x_{Q_{\tau}^{k}}$ the center of the cube $Q_{\tau}^{k} \in \mathcal{D}$. We also denote

$$
\mathcal{D}_{v}:=\left\{Q_{\tau}^{v+1} \in \mathcal{D}: \tau \in I_{v+1}\right\}
$$

for each $v \in \mathbb{Z}$. Then for $Q \in \mathcal{D}_{v}$, we have $B\left(x_{Q}, c_{0} 2^{-v}\right) \subset Q \subset B\left(x_{Q}, \kappa_{0} 2^{-v}\right)=$ : $B_{Q}$, where $c_{0}$ is a constant independent of $Q$.

2.2. Kernel estimates. Denote by $E_{L}(\lambda)$ a spectral decomposition of $L$. Then by spectral theory, for any bounded Borel function $F:[0, \infty) \rightarrow \mathbb{C}$, we can define

$$
F(L)=\int_{0}^{\infty} F(\lambda) d E_{L}(\lambda)
$$

as a bounded operator on $L^{2}(X)$. It is well known that the kernel $K_{\cos (t \sqrt{L})}$ of $\cos (t \sqrt{L})$ satisfies

$$
\operatorname{supp} K_{\cos (t \sqrt{L})} \subset\{(x, y) \in X \times X: d(x, y) \leqslant t\} .
$$

See, for example, [27].

We have the following useful lemma; see, for example, [44].

LEMMA 2.5. Let $\varphi \in \mathscr{S}(\mathbb{R})$ be an even function with $\operatorname{supp} \varphi \subset(-1,1)$ and $\int \varphi=2 \pi$. Denote by $\Phi$ the Fourier transform of $\varphi$. Then for every $k \in \mathbb{N}$, the kernel $K_{\left(t^{2} L\right)^{k} \Phi(t \sqrt{L})}$ of $\left(t^{2} L\right)^{k} \Phi(t \sqrt{L})$ satisfies

$$
\operatorname{supp} K_{\left(t^{2} L\right)^{k} \Phi(t \sqrt{L})} \subset\{(x, y) \in X \times X: d(x, y) \leqslant t\},
$$

and

$$
\left|K_{\left(t^{2} L\right)^{k} \Phi(t \sqrt{L})}(x, y)\right| \leqslant \frac{C}{V(x, t)} .
$$

The following lemma gives some kernel estimates which play an important role in the proof of our main results. 
LEMMA 2.6. (a) Let $\varphi \in \mathscr{S}(\mathbb{R})$ be an even function. Then for any $N>0$, there exists $C>0$ such that

$$
\left|K_{\varphi(t \sqrt{L})}(x, y)\right| \leqslant \frac{C}{V(x \vee y, t)}\left(1+\frac{d(x, y)}{t}\right)^{-N},
$$

for all $t>0$ and $x, y \in X$.

(b) Let $\varphi_{1}, \varphi_{2} \in \mathscr{S}(\mathbb{R})$ be even functions. Then for any $N>0$, there exists $C>0$ such that

$$
\left|K_{\varphi_{1}(t \sqrt{L}) \varphi_{2}(s \sqrt{L})}(x, y)\right| \leqslant C \frac{1}{V(x \vee y, t)}\left(1+\frac{d(x, y)}{t}\right)^{-N},
$$

for all $t \leqslant s<2 t$ and $x, y \in X$.

(c) Let $\varphi_{1}, \varphi_{2} \in \mathscr{S}(\mathbb{R})$ be even functions with $\varphi_{2}^{(\nu)}(0)=0$ for $v=0,1, \ldots, 2 \ell$ for some $\ell \in \mathbb{Z}^{+}$. Then for any $N>0$, there exists $C>0$ such that

$$
\left|K_{\varphi_{1}(t \sqrt{L}) \varphi_{2}(s \sqrt{L})}(x, y)\right| \leqslant C\left(\frac{s}{t}\right)^{2 \ell} \frac{1}{V(x \vee y, t)}\left(1+\frac{d(x, y)}{t}\right)^{-N},
$$

for all $t \geqslant s>0$ and $x, y \in X$.

Note that any function in $\mathscr{S}(\mathbb{R})$ with compact support in $(0, \infty)$ can be extended to an even function in $\mathscr{S}(\mathbb{R})$ with derivatives of all orders vanishing at 0 . Hence, the results in each part (a), (b) and (c) hold for such functions.

Proof. (a) The estimate (14) was proved in [25, Lemma 2.3] in the particular case $X=\mathbb{R}^{n}$, but the proof is still valid in the case of spaces of homogeneous type. For the items (b) and (c), we refer to [21].

REMARK 2.7. (i) From (3), the term $V(x \vee y, t)$ on the right-hand side of estimates in Lemma 2.6 can be replaced by $V(x \vee y, d(x, y))$. (ii) We will often use the following inequality:

$$
\left(1+\frac{d(x, y)}{t}\right)^{-N}\left(1+\frac{d(y, z)}{t}\right)^{-N} \lesssim\left(1+\frac{d(x, z)}{t}\right)^{-N}
$$

for all $x, y, z \in X$ and all $t, N>0$. We may use these in the sequel without stating any reasons. 
2.3. A new class of distributions. We fix $x_{0} \in X$ as a reference point in $X$. The class of test functions $\mathcal{S}$ associated with $L$ is defined as the set of all functions $\phi \in \bigcap_{m \geqslant 1} D\left(L^{m}\right)$ such that

$$
\mathcal{P}_{m, \ell}(\phi)=\sup _{x \in X}\left(1+d\left(x, x_{0}\right)\right)^{m}\left|L^{\ell} \phi(x)\right|<\infty, \quad \forall m>0, \ell \in \mathbb{N} .
$$

It was proved in [50] that $\mathcal{S}$ is a complete locally convex space with topology generated by the family of seminorms $\left\{\mathcal{P}_{m, \ell}: m>0, \ell \in \mathbb{N}\right\}$. As usual, we define the space of distributions $\mathcal{S}^{\prime}$ as the set of all continuous linear functional on $\mathcal{S}$ with the inner product defined by

$$
\langle f, \phi\rangle=f(\bar{\phi})
$$

for all $f \in \mathcal{S}^{\prime}$ and $\phi \in \mathcal{S}$.

The space of distributions $\mathcal{S}^{\prime}$ can be used to define the inhomogeneous Besov and Triebel-Lizorkin spaces. However, in order to study the homogeneous version of these spaces, we need some modifications. Following [36], we define the space $\mathcal{S}_{\infty}$ as the set of all functions $\phi \in \mathcal{S}$ such that for each $k \in \mathbb{N}$, there exists $g_{k} \in \mathcal{S}$ so that $\phi=L^{k} g_{k}$. Note that such an $g_{k}$, if exists, is unique; see [36]. The topology in $\mathcal{S}_{\infty}$ is generated by the following family of seminorms:

$$
\mathcal{P}_{m, \ell, k}^{*}(\phi)=\mathcal{P}_{m, \ell}\left(g_{k}\right), \quad \forall m>0 ; \ell, k \in \mathbb{N}
$$

where $\phi=L^{k} g_{k}$.

We then denote by $\mathcal{S}_{\infty}^{\prime}$ the set of all continuous linear functionals on $\mathcal{S}_{\infty}$.

In order to have an insightful understanding about the distributions in $\mathcal{S}_{\infty}^{\prime}$, we define

$$
\mathscr{P}_{m}=\left\{g \in \mathcal{S}^{\prime}: L^{m} g=0\right\}, \quad m \in \mathbb{N}
$$

and set $\mathscr{P}=\bigcup_{m \in \mathbb{N}} \mathscr{P}_{m}$.

From [36, Proposition 3.7], we have the following.

Proposition 2.8. The following identification is valid $\mathcal{S}^{\prime} / \mathscr{P}=\mathcal{S}_{\infty}^{\prime}$.

It was proved in [36] that with $L=-\Delta$, the Laplacian on $\mathbb{R}^{n}$, the distributions in $\mathcal{S}^{\prime} / \mathscr{P}=\mathcal{S}_{\infty}^{\prime}$ are identical with the classical tempered distributions modulo polynomial.

From Lemma 2.6, we can see that if $\varphi \in \mathscr{S}(\mathbb{R})$ with $\operatorname{supp} \varphi \subset(0, \infty)$, then we have $K_{\varphi(t \sqrt{L})}(x, \cdot) \in \mathcal{S}_{\infty}$ and $K_{\varphi(t \sqrt{L})}(\cdot, y) \in \mathcal{S}_{\infty}$. Therefore, we can define

$$
\varphi(t \sqrt{L}) f(x)=\left\langle f, K_{\varphi(t \sqrt{L})}(x, \cdot)\right\rangle
$$

for all $f \in \mathcal{S}_{\infty}^{\prime}$. 
The support condition $\operatorname{supp} \varphi \subset(0, \infty)$ is essential to be able to define $\varphi(t \sqrt{L}) f$ with $f \in \mathcal{S}_{\infty}^{\prime}$. In general, if $\varphi \in \mathscr{S}(\mathbb{R})$, then we have $K_{\varphi(t \sqrt{L})}(x, \cdot) \in \mathcal{S}$ and $K_{\varphi(t \sqrt{L})}(\cdot, y) \in \mathcal{S}$. In this situation, it is possible to define $\varphi(t \sqrt{L}) f$ when $f \in \mathcal{S}^{\prime}$, but it is not clear how to define $\varphi(t \sqrt{L}) f$ when $f \in \mathcal{S}_{\infty}^{\prime}$.

Lemma 2.9. Let $f \in \mathcal{S}^{\prime}$ and $\varphi \in \mathscr{S}(\mathbb{R})$ be an even function. Then there exist $m>0$ and $K>0$ such that

$$
|\varphi(t \sqrt{L}) f(x)| \lesssim \frac{\left(t \vee t^{-1}\right)^{m}}{V\left(x_{0}, t\right)}\left(1+d\left(x, x_{0}\right)\right)^{K} .
$$

The similar estimate holds true if $f \in \mathcal{S}_{\infty}^{\prime}$ and $\varphi \in \mathscr{S}(\mathbb{R})$ supported in $[1 / 2,2]$.

Proof. Since $\varphi(t \sqrt{L})(x, \cdot) \in \mathcal{S}$, we have

$$
|\varphi(t \sqrt{L}) f(x)| \lesssim \mathcal{P}_{m^{\prime}, \ell}\left(K_{\varphi(t \sqrt{L})}(x, \cdot)\right)
$$

for some $m^{\prime} \in \mathbb{N}$ and $\ell \geqslant 0$.

Using the kernel estimates in Lemma 2.6 and simple calculations, we can find $m, K>0$ so that

$$
P_{m^{\prime}, \ell}\left(K_{\varphi(t \sqrt{L})}(x, \cdot)\right) \lesssim \frac{\left(t \vee t^{-1}\right)^{m}}{V\left(x_{0}, t\right)}\left(1+d\left(x, x_{0}\right)\right)^{m} .
$$

This implies (19).

The proof is similar when $f \in \mathcal{S}_{\infty}^{\prime}$ and $\varphi \in \mathscr{S}(\mathbb{R})$ supported in $[1 / 2,2]$. We omit the details.

2.4. Calderón reproducing formulas. In what follows, by a 'partition of unity', we shall mean a function $\psi \in \mathcal{S}(\mathbb{R})$ such that $\operatorname{supp} \psi \subset[1 / 2,2]$, $\int \psi(\xi) \frac{d \xi}{\xi} \neq 0$ and

$$
\sum_{j \in \mathbb{Z}} \psi_{j}(\lambda)=1 \quad \text { on }(0, \infty)
$$

where $\psi_{j}(\lambda):=\psi\left(2^{-j} \lambda\right)$ for each $j \in \mathbb{Z}$.

Proposition 2.10. Let $\psi$ be a partition of unity. Then for any $f \in \mathcal{S}_{\infty}^{\prime}$, we have

$$
f=\sum_{j \in \mathbb{Z}} \psi_{j}(\sqrt{L}) f \quad \text { in } \mathcal{S}_{\infty}^{\prime}
$$


Proof. By duality, it suffices to prove that for each $f \in \mathcal{S}_{\infty}$,

$$
f=\sum_{j \in \mathbb{Z}} \psi_{j}(\sqrt{L}) f \quad \text { in } \mathcal{S}_{\infty} .
$$

Indeed, since $f \in \mathcal{S}_{\infty}$, for each $k \in \mathbb{N}$, there exists $g_{k} \in \mathcal{S}$ so that $f=L^{k} g_{k}$.

For $m>0$ and $\ell, k \in \mathbb{N}$, we have

$$
\mathcal{P}_{m, \ell, k}^{*}\left(\psi_{j}(\sqrt{L}) f\right)=\sup _{x \in X}\left(1+d\left(x, x_{0}\right)\right)^{m}\left|L^{\ell} \psi_{j}(\sqrt{L}) g_{k}(x)\right| .
$$

If $j \geqslant 0$, then we have

$$
\left(1+d\left(x, x_{0}\right)\right)^{m}\left|L^{\ell} \psi_{j}(\sqrt{L}) f(x)\right|=\left(1+d\left(x, x_{0}\right)\right)^{m}\left|2^{-2 j} \tilde{\psi}_{j}(\sqrt{L}) L^{\ell+1} g_{k}(x)\right|,
$$

where $\tilde{\psi}(\xi)=\xi^{-2} \psi(\xi)$.

This, along with Lemmas 2.6 and 2.2, implies that

$$
\begin{aligned}
(1 & \left.+d\left(x, x_{0}\right)\right)^{m}\left|L^{\ell} \psi_{j}(\sqrt{L}) f(x)\right| \\
& \lesssim 2^{-2 j}\left(1+d\left(x, x_{0}\right)\right)^{m} \int_{X} \frac{1}{V\left(x, 2^{-j}\right)}\left(1+\frac{d(x, y)}{2^{-j}}\right)^{-N-m}\left|L^{\ell+1} g_{k}(y)\right| d \mu(y) \\
& \lesssim 2^{-2 j} \int_{X} \frac{1}{V\left(x, 2^{-j}\right)}\left(1+\frac{d(x, y)}{2^{-j}}\right)^{-N}\left(1+d\left(y, x_{0}\right)\right)^{m}\left|L^{\ell+1} g_{k}(y)\right| d \mu(y) \\
& \lesssim 2^{-2 j} \mathcal{P}_{m, \ell+1}\left(g_{k}\right) \int_{X} \frac{1}{V\left(x, 2^{-j}\right)}\left(1+\frac{d(x, y)}{2^{-j}}\right)^{-N} d \mu(y) \\
& \lesssim 2^{-2 j} \mathcal{P}_{m, \ell+1, k}^{*}(f)
\end{aligned}
$$

as long as $N>n$.

Hence,

$$
\mathcal{P}_{m, \ell, k}^{*}\left(\psi_{j}(\sqrt{L}) f\right) \lesssim 2^{-2 j} \mathcal{P}_{m, \ell+1, k}^{*}(f), \quad \forall j \geqslant 0 .
$$

If $j<0$, then we have

$$
\left(1+d\left(x, x_{0}\right)\right)^{m}\left|L^{\ell} \psi_{j}(\sqrt{L}) f(x)\right|=2^{2 j(\ell+k+m+1)}\left(1+d\left(x, x_{0}\right)\right)^{m}\left|\varphi_{j}(\sqrt{L}) g_{k+m+1}(x)\right|,
$$

where $\varphi(\lambda)=\lambda^{2(\ell+k+m+1)} \psi(\lambda)$.

Arguing similarly to (20), we obtain

$$
\mathcal{P}_{m, \ell, k}^{*}\left(\psi_{j}(\sqrt{L}) f\right) \lesssim 2^{2 j} \mathcal{P}_{m, 0, k+m+1}^{*}(f), \quad \forall j<0 .
$$

From (21), (22) and the fact that $\mathcal{S}_{\infty}$ is complete, we deduce that there exists $h \in \mathcal{S}_{\infty}$ so that

$$
h=\sum_{j \in \mathbb{Z}} \psi_{j}(\sqrt{L}) f \quad \text { in } \mathcal{S}_{\infty}
$$


On the other hand, by spectral theory, we have

$$
f=\sum_{j \in \mathbb{Z}} \psi_{j}(\sqrt{L}) f \quad \text { in } L^{2}(X) .
$$

Therefore, $f \equiv h$, and this concludes the proposition.

PROPOSITION 2.11. Let $\psi \in \mathscr{S}(\mathbb{R})$ be such that $\operatorname{supp} \psi \subset[1 / 2,2]$ and $\int \psi \frac{d \xi}{\xi}$ $\neq 0$. Then for any $f \in \mathcal{S}_{\infty}^{\prime}$, we have

$$
f=c_{\psi} \int_{0}^{\infty} \psi(t \sqrt{L}) f \frac{d t}{t} \quad \text { in } \mathcal{S}_{\infty}^{\prime}
$$

where $c_{\psi}=\left[\int_{0}^{\infty} \psi(t) \frac{d t}{t}\right]^{-1}$.

Moreover, if $f \in \mathcal{S}^{\prime}$, then there exists $\rho \in \mathscr{P}$ so that

$$
f-\rho=c_{\psi} \int_{0}^{\infty} \psi(t \sqrt{L}) f \frac{d t}{t} \quad \text { in } \mathcal{S}^{\prime} .
$$

Proof. We first show that

$$
f=c_{\psi} \int_{0}^{\infty} \psi(t \sqrt{L}) f \frac{d t}{t} \text { in } \mathcal{S}_{\infty} .
$$

Arguing similarly to the proof of Proposition 2.10, we can prove that

$$
\lim _{N \rightarrow \infty} \int_{0}^{1 / N} \psi(t \sqrt{L}) f \frac{d t}{t}=\lim _{N \rightarrow \infty} \int_{N}^{\infty} \psi(t \sqrt{L}) f \frac{d t}{t}=0 \quad \text { in } \mathcal{S}_{\infty},
$$

and, consequently, by using the completeness of $\mathcal{S}_{\infty}$ and the spectral theory as in the proof of Proposition 2.10, we obtain the representation

$$
f=c_{\psi} \int_{0}^{\infty} \psi(t \sqrt{L}) f \frac{d t}{t} \text { in } \mathcal{S}_{\infty} .
$$

It follows by duality that

$$
f=c_{\psi} \int_{0}^{\infty} \psi(t \sqrt{L}) f \frac{d t}{t} \quad \text { in } \mathcal{S}_{\infty}^{\prime} .
$$

For the second part, we note that for $g \in \mathcal{S}$, we have

$$
\begin{aligned}
\int_{0}^{\infty} \psi(s \sqrt{L}) g \frac{d s}{s} & =\int_{0}^{1} \psi(s \sqrt{L}) g \frac{d s}{s}+\int_{1}^{\infty} \psi(s \sqrt{L}) g \frac{d s}{s} \\
& =\int_{0}^{1} \psi(s \sqrt{L}) g \frac{d s}{s}+\tilde{\psi}(\sqrt{L}) g,
\end{aligned}
$$

where $\tilde{\psi}(x)=\int_{|x|}^{\infty} \psi(s) \frac{d s}{s}=\int_{1}^{\infty} \psi(s x) \frac{d s}{s}$. 
Hence, for any $\ell \in \mathbb{N}, k>0$ and $x \in X$, we have

$$
\begin{aligned}
(1+ & \left.d\left(x, x_{0}\right)\right)^{k}\left|L^{\ell}\left(\int_{0}^{\infty} \psi(s \sqrt{L}) g(x) \frac{d s}{s}\right)\right| \\
\leqslant & \left(1+d\left(x, x_{0}\right)\right)^{k} \int_{0}^{1}\left|\psi(s \sqrt{L}) L^{\ell} g(x)\right| \frac{d s}{s} \\
& +\left(1+d\left(x, x_{0}\right)\right)^{k}\left|\tilde{\psi}(\sqrt{L}) L^{\ell} g(x)\right| \\
\leqslant & \left(1+d\left(x, x_{0}\right)\right)^{k} \int_{0}^{1} s^{N}\left|(s \sqrt{L})^{-2 N} \psi(s \sqrt{L}) L^{\ell+N} g(x)\right| \frac{d s}{s} \\
& +\left(1+d\left(x, x_{0}\right)\right)^{k}\left|\tilde{\psi}(\sqrt{L}) L^{\ell} g(x)\right| \\
= & : I_{1}(g)+I_{2}(g),
\end{aligned}
$$

where $N$ is a fixed number which is greater than $k+n$.

Note that $x^{-2 N} \psi(x)$ is a function in $\mathscr{S}(\mathbb{R})$ supported in $[1 / 2,2]$. Using Lemma 2.6 and arguing similarly to the proof of Proposition 2.10, we can show that

$$
I_{1}(g) \lesssim \mathcal{P}_{k, \ell+N}(g) .
$$

For the same reason, since $\tilde{\psi}$ is an even function in $\mathscr{S}(\mathbb{R})$, we also have

$$
I_{2}(g) \lesssim \mathcal{P}_{k, \ell}(g)
$$

As a consequence,

$$
\left(1+d\left(x, x_{0}\right)\right)^{k}\left|L^{\ell}\left(\int_{0}^{\infty} \psi(s \sqrt{L}) g(x) \frac{d s}{s}\right)\right| \lesssim \mathcal{P}_{k, \ell+N}(g)+\mathcal{P}_{k, \ell}(g)
$$

for all $\ell \in \mathbb{N}, k>0$ and $x \in X$.

This implies that $\int_{0}^{\infty} \psi(s \sqrt{L}) g \frac{d s}{s} \in \mathcal{S}$ whenever $g \in \mathcal{S}$. By duality, $\int_{0}^{\infty} \psi(s \sqrt{L}) f \frac{d s}{s} \in \mathcal{S}^{\prime}$ whenever $f \in \mathcal{S}^{\prime}$. This, along with (23) and the fact that $\mathcal{S}_{\infty}^{\prime}=\mathcal{S}^{\prime} / \mathscr{P}$, implies that there exists $\rho \in \mathscr{P}$ so that

$$
f-\rho=c_{\psi} \int_{0}^{\infty} \psi(s \sqrt{L}) f \frac{d s}{s}
$$

in $\mathcal{S}^{\prime}$.

This completes our proof.

LEMMA 2.12. Let $\phi \in \mathscr{S}(\mathbb{R})$ be an even function such that $\phi(\xi) \neq 0$ on $(-2$, $-1 / 2) \cup(1 / 2,2)$. Then there exist $a, b, c>0$ and even functions $\Psi, \psi \in \mathscr{S}(\mathbb{R})$ 
with $\operatorname{supp} \Psi \subset[-a, a]$, supp $\psi \subset[-c,-b] \cup[b, c]$ so that for every $f \in \mathcal{S}^{\prime}$ and every $j \in \mathbb{Z}$ and $t \in[1,2]$, we have

$$
f=\Psi\left(2^{-j} t \sqrt{L}\right) f+\sum_{k \geqslant 1} \phi\left(2^{-(k+j)} t \sqrt{L}\right) \psi\left(2^{-(k+j)} t \sqrt{L}\right) f \quad \text { in } \mathcal{S}^{\prime} .
$$

Proof. It is well known that there exists an even function $\psi \in \mathscr{S}(\mathbb{R})$ with supp $\psi \subset[-c,-b] \cup[b, c]$ for some $c>b>0$ so that

$$
\sum_{k=-\infty}^{\infty} \phi\left(2^{-k} \lambda\right) \psi\left(2^{-k} \lambda\right)=1, \quad \forall \lambda \neq 0 .
$$

See, for example, [58]. Define $\Psi \in \mathscr{S}(\mathbb{R})$ by $\Psi(0)=1$, and $\Psi(\lambda)=$ $\sum_{k \leqslant 0} \phi\left(2^{-k} \lambda\right) \psi\left(2^{-k} \lambda\right)$ for $\lambda \neq 0$. Then it is easy to see that $\operatorname{supp} \Psi \subset[-a, a]$ for some $a>0$ and that

$$
\Psi(\lambda)+\sum_{k \geqslant 1} \phi\left(2^{-k} \lambda\right) \psi\left(2^{-k} \lambda\right)=1, \quad \forall \lambda \in \mathbb{R} .
$$

Using the above identity and arguing similarly to the proof of Proposition 2.10, we conclude that

$$
f=\Phi\left(2^{-j} t \sqrt{L}\right) \Psi\left(2^{-j} t \sqrt{L}\right) f+\sum_{k \geqslant 1} \phi\left(2^{-(k+j)} t \sqrt{L}\right) \psi\left(2^{-(k+j)} t \sqrt{L}\right) f \quad \text { in } \mathcal{S}^{\prime} .
$$

(A close inspection of the proof in [58] shows that we can take $a=2$, and $1 / 2<$ $b<c / 2<1$.)

2.5. Maximal function estimates. We begin with some technical estimates.

LEMmA 2.13. Let $\psi, \varphi \in \mathscr{S}(\mathbb{R})$ be even functions. Assume that $0<a \leqslant b<\infty$ and $\lambda \geqslant 0$. Then there exists $C>0$ such that

$$
\begin{gathered}
\sup _{y \in X}|\psi(s \sqrt{L}) \varphi(t \sqrt{L}) f(y)|\left(1+\frac{d(x, y)}{t}\right)^{-\lambda} \\
\leqslant C \sup _{y \in X}|\varphi(t \sqrt{L}) f(y)|\left(1+\frac{d(x, y)}{t}\right)^{-\lambda}
\end{gathered}
$$

for all $f \in \mathcal{S}^{\prime}, x \in X$ and $s \in[a t, b t]$. If both functions $\psi$ and $\varphi$ are supported in $[1 / 2,2]$, then (25) holds for all $f \in \mathcal{S}_{\infty}^{\prime}$. 
Proof. From Lemma 2.6, for $N>n$ and $f \in \mathcal{S}^{\prime}$, we have

$$
\begin{aligned}
\mid \psi & (s \sqrt{L}) \varphi(t \sqrt{L}) f(y) \mid\left(1+\frac{d(x, y)}{t}\right)^{-\lambda} \\
& \lesssim\left(1+\frac{d(x, y)}{t}\right)^{-\lambda} \int_{X} \frac{1}{V(y, s)}\left(1+\frac{d(y, z)}{s}\right)^{-N-\lambda}|\varphi(t \sqrt{L}) f(z)| d \mu(z) \\
& \sim\left(1+\frac{d(x, y)}{t}\right)^{-\lambda} \int_{X} \frac{1}{V(y, t)}\left(1+\frac{d(y, z)}{t}\right)^{-N-\lambda}|\varphi(t \sqrt{L}) f(z)| d \mu(z) \\
& \lesssim \int_{X} \frac{1}{V(y, t)}\left(1+\frac{d(y, z)}{t}\right)^{-N}\left(1+\frac{d(x, z)}{t}\right)^{-\lambda}|\varphi(t \sqrt{L}) f(z)| d \mu(z) .
\end{aligned}
$$

This, along with Lemma 2.2, implies the desired estimate.

Note that in the case that both functions $\psi$ and $\varphi$ are supported in [1/2,2], then we can define $\psi(s \sqrt{L}) \varphi(t \sqrt{L}) f$ for all $f \in \mathcal{S}_{\infty}^{\prime}$. The above argument also gives (25) in this case.

This completes our proof.

LEMmA 2.14. Let $\psi \in \mathscr{S}(\mathbb{R})$ with $\operatorname{supp} \psi \subset[1 / 2,2]$ and $\int \psi \frac{d \xi}{\xi} \neq 0$. Then for any $r>0$ and $N>0$, we have

$$
\begin{aligned}
& |\psi(t \sqrt{L}) f(x)|^{r} \\
& \quad \lesssim \int_{0}^{\infty} \int_{X} \frac{1}{V(x, s)}\left(1+\frac{d(x, y)}{s}\right)^{-N r}\left(\frac{s}{t} \wedge \frac{t}{s}\right)^{N r}|\psi(s \sqrt{L}) f(y)|^{r} d \mu(y) \frac{d s}{s}
\end{aligned}
$$

for all $f \in \mathcal{S}_{\infty}^{\prime}, x \in X$ and $t>0$.

Proof. By Proposition 2.11, we have

$$
\psi(u \sqrt{L}) f=c_{\psi} \int_{0}^{\infty} \psi(u \sqrt{L}) \psi(s \sqrt{L}) f \frac{d s}{s} \quad \text { pointwise, }
$$

where $c_{\psi}=\left[\int_{0}^{\infty} \psi(\xi) \frac{d \xi}{\xi}\right]^{-1}$.

This, along with the fact that supp $\psi \subset[1 / 2,2]$, yields

$$
\psi(u \sqrt{L}) f=c_{\psi} \int_{u / 4}^{4 u} \psi(u \sqrt{L}) \psi(s \sqrt{L}) f \frac{d s}{s} .
$$

Applying Lemma 2.6, we deduce that, for $N>0$,

$$
|\psi(u \sqrt{L}) f(y)| \lesssim \int_{u / 4}^{4 u} \int_{X} \frac{1}{V(y, u)}\left(1+\frac{d(y, z)}{u}\right)^{-N}|\psi(s \sqrt{L}) f(z)| d \mu(z) \frac{d s}{s}
$$




$$
\lesssim \int_{u / 4}^{4 u} \int_{X} \frac{1}{V(y, s)}\left(1+\frac{d(y, z)}{s}\right)^{-N}\left(\frac{s}{u}\right)^{N}|\psi(s \sqrt{L}) f(z)| d \mu(z) \frac{d s}{s}
$$

If $r \in[1, \infty)$, then using (28) for $A+N$, where $A>n$, together with Hölder's inequality and Lemma 2.2, we obtain (26).

We now consider the case $r \in(0,1)$. For each $x \in X$ and $t>0$, we define

$$
\psi_{N}^{* *}(t \sqrt{L}) f(x)=\sup _{y \in X} \sup _{s>0} \frac{|\psi(s \sqrt{L}) f(y)|}{\left(1+\frac{d(x, y)}{s}\right)^{N}}\left(\frac{s}{t} \wedge \frac{t}{s}\right)^{N} .
$$

Note that by Lemma 2.6, (28) holds with $V(y, s)$ replaced by $V(z, s)$. It follows that

$$
\begin{aligned}
\mid \psi & (u \sqrt{L}) f(y) \mid\left(1+\frac{d(x, y)}{u}\right)^{-N}\left(\frac{u}{t} \wedge \frac{t}{u}\right)^{N} \\
& \lesssim \int_{u / 4}^{4 u} \int_{X} \frac{1}{V(z, s)}\left(1+\frac{d(y, z)}{s}\right)^{-N}\left(1+\frac{d(x, y)}{u}\right)^{-N}\left(\frac{u}{t} \wedge \frac{t}{u}\right)^{N} \\
& \times|\psi(s \sqrt{L}) f(z)| d \mu(z) \frac{d s}{s} \\
\sim & \int_{u / 4}^{4 u} \int_{X} \frac{1}{V(z, s)}\left(1+\frac{d(y, z)}{s}\right)^{-N}\left(1+\frac{d(x, y)}{s}\right)^{-N}\left(\frac{s}{t} \wedge \frac{t}{s}\right)^{N} \\
& \times|\psi(s \sqrt{L}) f(z)| d \mu(z) \frac{d s}{s} \\
\lesssim & \int_{u / 4}^{4 u} \int_{X} \frac{1}{V(z, s)}\left(1+\frac{d(x, z)}{s}\right)^{-N}\left(\frac{s}{t} \wedge \frac{t}{s}\right)^{N}|\psi(s \sqrt{L}) f(z)| d \mu(z) \frac{d s}{s} \\
\lesssim & \psi_{N}^{* *}(t \sqrt{L}) f(x)^{1-r} \int_{u / 4}^{4 u} \int_{X} \frac{1}{V(z, s)}\left(1+\frac{d(x, z)}{s}\right)^{-N r}\left(\frac{s}{t} \wedge \frac{t}{s}\right)^{N r} \\
& \times|\psi(s \sqrt{L}) f(z)|^{r} d \mu(z) \frac{d s}{s} .
\end{aligned}
$$

By (19), there exists $N_{f}>0$ such that $\psi_{N}^{* *}(t \sqrt{L}) f(x)<\infty$ for all $N \geqslant N_{f}$ and $x \in X$. For any such $N$, taking the supremum over $u>0$ and $y \in X$ in the left-hand side of (29), we obtain

$$
\begin{aligned}
\psi_{N}^{* *}(t \sqrt{L}) f(x)^{r} \leqslant & C(f, N) \int_{u / 4}^{4 u} \int_{X} \frac{1}{V(z, s)}\left(1+\frac{d(x, z)}{s}\right)^{-N r}\left(\frac{s}{t} \wedge \frac{t}{s}\right)^{N r} \\
& \times|\psi(s \sqrt{L}) f(z)|^{r} d \mu(z) \frac{d s}{s}
\end{aligned}
$$




$$
\begin{aligned}
\leqslant & C(f, N) \int_{0}^{\infty} \int_{X} \frac{1}{V(z, s)}\left(1+\frac{d(x, z)}{s}\right)^{-N r}\left(\frac{s}{t} \wedge \frac{t}{s}\right)^{N r} \\
& \times|\psi(s \sqrt{L}) f(z)|^{r} d \mu(z) \frac{d s}{s} .
\end{aligned}
$$

Consequently,

$$
\begin{aligned}
& |\psi(t \sqrt{L}) f(x)|^{r} \\
& \leqslant \\
& \quad C(f, N) \int_{0}^{\infty} \int_{X} \frac{1}{V(z, s)}\left(1+\frac{d(x, z)}{s}\right)^{-N r}\left(\frac{s}{t} \wedge \frac{t}{s}\right)^{N r} \\
& \quad \times|\psi(s \sqrt{L}) f(z)|^{r} d \mu(z) \frac{d s}{s} .
\end{aligned}
$$

Since the integral on the right-hand side of the above gets larger when $N$ gets smaller, (30) holds true for all $N>0$ and all $x \in X$. It follows that

$$
\begin{aligned}
|\psi(u \sqrt{L}) f(y)|^{r} \leqslant & C(f, N) \int_{0}^{\infty} \int_{X} \frac{1}{V(z, s)}\left(1+\frac{d(y, z)}{s}\right)^{-2 N r}\left(\frac{s}{u} \wedge \frac{u}{s}\right)^{2 N r} \\
& \times|\psi(s \sqrt{L}) f(z)|^{r} d \mu(z) \frac{d s}{s} \\
\leqslant & C(f, N) \int_{0}^{\infty} \int_{X} \frac{1}{V(z, s)}\left(1+\frac{d(y, z)}{s}\right)^{-N r}\left(\frac{s}{u} \wedge \frac{u}{s}\right)^{2 N r} \\
& \times|\psi(s \sqrt{L}) f(z)|^{r} d \mu(z) \frac{d s}{s}
\end{aligned}
$$

for all $N>0, u>0$ and $y \in X$.

Using the obvious inequalities

$$
\left(\frac{u}{t} \wedge \frac{t}{u}\right)^{A r}\left(\frac{s}{u} \wedge \frac{u}{s}\right)^{A r} \leqslant\left(\frac{s}{t} \wedge \frac{t}{s}\right)^{A r}
$$

and

$$
\left(1+\frac{d(y, z)}{s}\right)^{-A r}\left(1+\frac{d(x, y)}{u}\right)^{-A r}\left(\frac{s}{u} \wedge \frac{u}{s}\right)^{A r} \lesssim\left(1+\frac{d(x, z)}{s}\right)^{-A r},
$$

where $A=N+\tilde{n} / r$, we obtain

$$
\frac{|\psi(u \sqrt{L}) f(y)|^{r}}{\left(1+\frac{d(x, y)}{u}\right)^{A r}}\left(\frac{u}{t} \wedge \frac{t}{u}\right)^{A r}
$$




$$
\begin{aligned}
\leqslant & C(f, N, r) \int_{0}^{\infty} \int_{X} \frac{1}{V(z, s)}\left(1+\frac{d(x, z)}{s}\right)^{-A r}\left(\frac{s}{t} \wedge \frac{t}{s}\right)^{A r} \\
& \times|\psi(s \sqrt{L}) f(z)|^{r} d \mu(z) \frac{d s}{s} .
\end{aligned}
$$

Taking the supremum over all $u>0$ and $y \in X$ gives

$$
\begin{aligned}
\psi_{A}^{* *}(t \sqrt{L}) f(x)^{r} \leqslant & C(f, N, r) \int_{0}^{\infty} \int_{X} \frac{1}{V(z, s)}\left(1+\frac{d(x, z)}{s}\right)^{-A r}\left(\frac{s}{t} \wedge \frac{t}{s}\right)^{A r} \\
& \times|\psi(s \sqrt{L}) f(z)|^{r} d \mu(z) \frac{d s}{s} \\
\leqslant & C(f, N, r) \int_{0}^{\infty} \int_{X} \frac{1}{V(x, s)}\left(1+\frac{d(x, z)}{s}\right)^{-N r}\left(\frac{s}{t} \wedge \frac{t}{s}\right)^{N r} \\
& \times|\psi(s \sqrt{L}) f(z)|^{r} d \mu(z) \frac{d s}{s}
\end{aligned}
$$

for all $N>0, x \in X$ and $t>0$, where we use (3) in the last inequality.

Therefore, if the integral on the right-hand side of the above is finite, then $\psi_{N+\tilde{n} / r}^{* *}(t \sqrt{L}) f(x)<\infty$. In this case, we repeat the arguments in the first part of the proof and obtain the required inequality (26), where the constant in the inequality depends on $N$ and $r$ (but independent of $f$ ). On the other hand, if this integral is infinite, then (26) holds trivially. Thus, we have proved (26) for all $N>0$.

REMARK 2.15. Actually, we have proved a stronger statement:

$$
\begin{aligned}
\psi_{N+\tilde{n} / r}^{* *}(t \sqrt{L}) f(x)^{r} \leqslant & C_{N, r} \int_{0}^{\infty} \int_{X} \frac{1}{V(x, s)}\left(1+\frac{d(x, z)}{s}\right)^{-N r}\left(\frac{s}{t} \wedge \frac{t}{s}\right)^{N r} \\
& \times|\psi(s \sqrt{L}) f(z)|^{r} d \mu(z) \frac{d s}{s}
\end{aligned}
$$

for all $N>0, f \in \mathcal{S}_{\infty}^{\prime}, x \in X$ and $t>0$. Moreover, a close inspection of the proof of the lemma shows that we also have

$$
\begin{aligned}
\psi_{N}^{* *}(t \sqrt{L}) f(x)^{r} \leqslant & C_{N, r} \int_{0}^{\infty} \int_{X} \frac{1}{V(z, s)}\left(1+\frac{d(x, z)}{s}\right)^{-N r}\left(\frac{s}{t} \wedge \frac{t}{s}\right)^{N r} \\
& \times|\psi(s \sqrt{L}) f(z)|^{r} d \mu(z) \frac{d s}{s}
\end{aligned}
$$

for all $N>0, f \in \mathcal{S}_{\infty}^{\prime}, x \in X$ and $t>0$. 
For $\lambda>0, j \in \mathbb{Z}$ and $\varphi \in \mathscr{S}(\mathbb{R})$, the Peetre-type maximal function is defined, for $f \in \mathcal{S}^{\prime}$, by

$$
\varphi_{j, \lambda}^{*}(\sqrt{L}) f(x)=\sup _{y \in X} \frac{\left|\varphi_{j}(\sqrt{L}) f(y)\right|}{\left(1+2^{j} d(x, y)\right)^{\lambda}}, \quad x \in X,
$$

where $\varphi_{j}(\lambda)=\varphi\left(2^{-j} \lambda\right)$.

Obviously, we have

$$
\varphi_{j, \lambda}^{*}(\sqrt{L}) f(x) \geqslant\left|\varphi_{j}(\sqrt{L}) f(x)\right|, \quad x \in X .
$$

Similarly, for $s, \lambda>0$, we set

$$
\varphi_{\lambda}^{*}(s \sqrt{L}) f(x)=\sup _{y \in X} \frac{|\varphi(s \sqrt{L}) f(y)|}{(1+d(x, y) / s)^{\lambda}}, \quad f \in \mathcal{S}^{\prime} .
$$

We note that in the particular case when $\varphi$ is supported in $(0, \infty)$, these maximal functions can defined for $f \in \mathcal{S}_{\infty}^{\prime}$ via (18).

Due to (19), $\varphi_{\lambda}^{*}(s \sqrt{L}) f(x)<\infty$ for all $x \in X$, provided that $\lambda$ is sufficiently large.

Proposition 2.16. Let $\psi \in \mathscr{S}(\mathbb{R})$ with $\operatorname{supp} \psi \subset[1 / 2,2]$ and $\varphi \in \mathscr{S}(\mathbb{R})$ be a partition of unity. Then for any $\lambda>0$ and $j \in \mathbb{Z}$, we have

$$
\sup _{s \in\left[2^{-j-1}, 2^{-j}\right]} \psi_{\lambda}^{*}(s \sqrt{L}) f(x) \lesssim \sum_{k=j-2}^{j+3} \varphi_{k, \lambda}^{*}(\sqrt{L}) f(x),
$$

for all $f \in \mathcal{S}_{\infty}^{\prime}$ and $x \in X$.

Proof. Fix $j \in \mathbb{Z}$ and $s \in\left[2^{-j-1}, 2^{-j}\right]$. First note that

$$
\psi(s \sqrt{L})=\sum_{k=j-2}^{j+3} \psi(s \sqrt{L}) \varphi_{k}(\sqrt{L}) .
$$

Since $2^{-j} \sim s$, by Lemma 2.6, we have, for $y \in X$ and $N>n$,

$$
\begin{aligned}
& |\psi(s \sqrt{L}) f(y)| \leqslant \sum_{k=j-2}^{j+3}\left|\psi(s \sqrt{L}) \varphi_{k}(\sqrt{L}) f(y)\right| \\
& \quad \lesssim \sum_{k=j-2}^{j+3} \int_{X} \frac{1}{V\left(y, 2^{-j}\right)}\left(1+2^{j} d(y, z)\right)^{-N-\lambda}\left|\varphi_{k}(\sqrt{L}) f(z)\right| d \mu(z) .
\end{aligned}
$$


It follows that

$$
\begin{aligned}
\frac{|\psi(s \sqrt{L}) f(y)|}{(1+d(x, y) / s)^{\lambda}} \lesssim & \sum_{k=j-2}^{j+3} \int_{X} \frac{1}{V\left(y, 2^{-j}\right)}\left(1+2^{j} d(y, z)\right)^{-N-\lambda} \\
& \times \frac{\left|\varphi_{k}(\sqrt{L}) f(z)\right|}{\left(1+2^{j} d(x, y)\right)^{\lambda}} d \mu(z) \\
\lesssim & \sum_{k=j-2}^{j+3} \int_{X} \frac{1}{V\left(y, 2^{-j}\right)}\left(1+2^{j} d(y, z)\right)^{-N} \\
& \times \frac{\left|\varphi_{k}(\sqrt{L}) f(z)\right|}{\left(1+2^{j} d(x, y)\right)^{\lambda}\left(1+2^{j} d(y, z)\right)^{\lambda}} d \mu(z) \\
\lesssim & \sum_{k=j-2}^{j+3} \int_{X} \frac{1}{V\left(y, 2^{-j}\right)}\left(1+2^{j} d(y, z)\right)^{-N} \\
& \times \frac{\left|\varphi_{k}(\sqrt{L}) f(z)\right|}{\left(1+2^{j} d(x, z)\right)^{\lambda}} d \mu(z) .
\end{aligned}
$$

Using the fact that $2^{k} \sim 2^{j}$, we obtain

$$
\begin{aligned}
& \frac{|\psi(s \sqrt{L}) f(y)|}{(1+d(x, y) / s)^{\lambda}} \\
& \quad \lesssim \sum_{k=j-2}^{j+3} \int_{X} \frac{1}{V\left(y, 2^{-j}\right)}\left(1+2^{j} d(y, z)\right)^{-N} \frac{\left|\varphi_{k}(\sqrt{L}) f(z)\right|}{\left(1+2^{k} d(x, z)\right)^{\lambda}} d \mu(z) \\
& \quad \lesssim \sum_{k=j-2}^{j+3} \varphi_{k, \lambda}^{*}(\sqrt{L}) f(x) \int_{X} \frac{1}{V\left(y, 2^{-j}\right)}\left(1+2^{j} d(y, z)\right)^{-N} d \mu(z) \\
& \quad \lesssim \sum_{k=j-2}^{j+3} \varphi_{k, \lambda}^{*}(\sqrt{L}) f(x),
\end{aligned}
$$

where, in the last inequality, we use Lemma 2.2. Taking the supremum over $y \in X$, we derive (36).

Proposition 2.17. Let $\psi$ be a partition of unity. Then for any $\lambda, s>0$ and $r>0$, we have the following:

$$
\psi_{\lambda}^{*}(s \sqrt{L}) f(x) \lesssim\left[\int_{X} \frac{1}{V(z, s)} \frac{|\psi(s \sqrt{L}) f(z)|^{r}}{(1+d(x, z) / s)^{\lambda r}} d \mu(z)\right]^{1 / r},
$$




$$
\psi_{\lambda+\tilde{n} / r}^{*}(s \sqrt{L}) f(x) \lesssim\left[\int_{X} \frac{1}{V(x, s)} \frac{|\psi(s \sqrt{L}) f(z)|^{r}}{(1+d(x, z) / s)^{\lambda r}} d \mu(z)\right]^{1 / r}
$$

for all $f \in \mathcal{S}_{\infty}^{\prime}$ and $x \in X$.

Proof. We start with the proof of (41). Similarly to (27), we have

$$
\psi(s \sqrt{L}) f=c_{\psi} \int_{s / 4}^{4 s} \psi(u \sqrt{L}) \psi(s \sqrt{L}) f \frac{d u}{u} .
$$

If $r \in(0,1)$, then using Lemma 2.6 and the fact $u \sim s$, we see that, for $\lambda>0$ and $x, y \in X$,

$$
\begin{aligned}
\frac{|\psi(s \sqrt{L}) f(y)|}{(1+d(x, y) / s)^{\lambda}} & \lesssim \int_{X} \frac{1}{V(z, s)}\left(1+\frac{d(y, z)}{s}\right)^{-\lambda} \frac{|\psi(s \sqrt{L}) f(z)|}{(1+d(x, y) / s)^{\lambda}} d \mu(z) \\
& \lesssim \int_{X} \frac{1}{V(z, s)} \frac{|\psi(s \sqrt{L}) f(z)|}{(1+d(x, z) / s)^{\lambda}} d \mu(z) \\
& \lesssim\left[\psi_{\lambda}^{*}(s \sqrt{L}) f(x)\right]^{1-r} \int_{X} \frac{1}{V(z, s)} \frac{|\psi(s \sqrt{L}) f(z)|^{r}}{(1+d(x, z) / s)^{\lambda r}} d \mu(z)
\end{aligned}
$$

By (19), there exists $\lambda_{f}>0$ such that $\psi_{\lambda}^{*}(s \sqrt{L}) f(x)<\infty$ for all $\lambda \geqslant \lambda_{f}, s>0$ and $x \in X$. Using a bootstrap argument similarly to the proof of Lemma 2.14 (see (32)), in which (29) is replaced by the above inequality, we deduce the required inequality (41) in this case. We omit the details.

On the other hand, if $r \geqslant 1$, we similarly have

$$
\begin{aligned}
& \frac{|\psi(s \sqrt{L}) f(y)|}{(1+d(x, y) / s)^{\lambda+\tilde{n} / r}} \\
& \quad \lesssim \int_{X} \frac{1}{V(z, s)}\left(1+\frac{d(y, z)}{s}\right)^{-n-\lambda-\tilde{n} / r} \frac{|\psi(s \sqrt{L}) f(z)|}{(1+d(x, y) / s)^{\lambda+\tilde{n} / r}} d \mu(z) \\
& \lesssim \int_{X} \frac{1}{V(z, s)}\left(1+\frac{d(y, z)}{s}\right)^{-n} \frac{|\psi(s \sqrt{L}) f(z)|}{(1+d(x, z) / s)^{\lambda+\tilde{n} / r}} d \mu(z) .
\end{aligned}
$$

We now apply Hölder's inequality, Lemma 2.2 and (3) to deduce that

$$
\begin{aligned}
\frac{|\psi(s \sqrt{L}) f(y)|}{(1+d(x, y) / s)^{\lambda+\tilde{n} / r}} & \lesssim\left[\int_{X} \frac{1}{V(z, s)} \frac{|\psi(s \sqrt{L}) f(z)|^{r}}{(1+d(x, z) / s)^{\lambda r+\tilde{n}}} d \mu(z)\right]^{1 / r} \\
& \lesssim\left[\int_{X} \frac{1}{V(x, s)} \frac{|\psi(s \sqrt{L}) f(z)|^{r}}{(1+d(x, z) / s)^{\lambda r}} d \mu(z)\right]^{1 / r} .
\end{aligned}
$$

This implies the required inequality (41) when $r \geqslant 1$. 
The proof of (40) can be done in a similar fashion, but slightly simpler, where (3) is not used.

Proposition 2.18. Let $\psi$ be a partition of unity and $\varphi \in \mathscr{S}(\mathbb{R})$ be an even function such that $\varphi \neq 0$ on $[1 / 2,2]$. Then for any $\lambda>0, j \in \mathbb{Z}$ and $r>0$, we have

$$
\left|\psi_{j}(\sqrt{L}) f(x)\right| \lesssim\left(\int_{2^{-j-2}}^{2^{-j+2}}\left|\varphi_{\lambda}^{*}(s \sqrt{L}) f(x)\right|^{r} \frac{d s}{s}\right)^{1 / r}
$$

for every $f \in \mathcal{S}^{\prime}$.

Proof. Since $\varphi \neq 0$ on $[1 / 2,2]$, there exists $\phi \in \mathscr{S}(\mathbb{R})$ supported in $[1 / 2,2]$ so that

$$
c_{\varphi, \phi}=\int_{0}^{\infty} \varphi(\xi) \phi(\xi) \frac{d \xi}{\xi} \neq 0 .
$$

It follows that, for each $j \in \mathbb{Z}$,

$$
\begin{aligned}
\psi_{j}(\sqrt{L}) f & =c_{\varphi, \phi}^{-1} \int_{0}^{\infty} \varphi(t \sqrt{L}) \phi(t \sqrt{L}) \psi_{j}(\sqrt{L}) f \frac{d \xi}{\xi} \\
& =c_{\varphi, \phi}^{-1} \int_{2^{j-2}}^{2^{j+2}} \varphi(t \sqrt{L}) \phi(t \sqrt{L}) \psi_{j}(\sqrt{L}) f \frac{d t}{t} .
\end{aligned}
$$

Hence, for any $r>0$,

$$
\left|\psi_{j}(\sqrt{L}) f(x)\right|^{r} \lesssim \sup _{t \in\left[2^{-j-2}, 2^{-j+2}\right]}\left|\varphi(t \sqrt{L}) \phi(t \sqrt{L}) \psi_{j}(\sqrt{L}) f(x)\right|^{r} .
$$

Fix $t \in\left[2^{-j-2}, 2^{-j+2}\right]$. By Lemma 2.14 and the fact that $\operatorname{supp} \phi \subset[1 / 2,2]$, we have, for $\lambda>0$,

$$
\begin{aligned}
\mid \phi( & t \sqrt{L})\left.\varphi(t \sqrt{L})\left[\psi_{j}(\sqrt{L}) f\right](x)\right|^{r} \\
\lesssim & \int_{0}^{\infty} \int_{X} \frac{1}{V(x, s)}\left(1+\frac{d(x, y)}{s}\right)^{-\lambda r}\left(\frac{s}{t} \wedge \frac{t}{s}\right)^{\lambda r} \\
& \times\left|\phi(s \sqrt{L}) \varphi(s \sqrt{L}) \psi_{j}(\sqrt{L}) f(y)\right|^{r} d \mu(y) \frac{d s}{s} \\
\lesssim & \int_{2^{-j-2}}^{2^{-j+2}} \int_{X} \frac{1}{V(x, s)}\left(1+\frac{d(x, y)}{s}\right)^{-\lambda r}\left(\frac{s}{t} \wedge \frac{t}{s}\right)^{\lambda r} \\
& \times\left|\phi(s \sqrt{L}) \varphi(s \sqrt{L}) \psi_{j}(\sqrt{L}) f(y)\right|^{r} d \mu(y) \frac{d s}{s} \\
\sim & \int_{2^{-j-2}}^{2^{-j+2}} \int_{X} \frac{1}{V(x, s)}\left(1+\frac{d(x, y)}{s}\right)^{-\lambda r}
\end{aligned}
$$




$$
\times\left|\phi(s \sqrt{L}) \varphi(s \sqrt{L}) \psi_{j}(\sqrt{L}) f(y)\right|^{r} d \mu(y) \frac{d s}{s} .
$$

Note that $s / 4 \leqslant 2^{-j} \leqslant 4 s$ when $2^{-j-2} \leqslant s \leqslant 2^{-j+2}$. Hence, applying Lemma 2.13, we see that

$$
\begin{aligned}
& \left(1+\frac{d(x, y)}{s}\right)^{-\lambda}\left|\phi(s \sqrt{L}) \varphi(s \sqrt{L}) \psi_{j}(\sqrt{L}) f(y)\right| \\
& \lesssim \sup _{y \in X}|\varphi(s \sqrt{L}) f(y)|\left(1+\frac{d(x, y)}{s}\right)^{-\lambda}=: \varphi_{\lambda}^{*}(s \sqrt{L}) f(x) .
\end{aligned}
$$

As a consequence,

$$
\sup _{t \in\left[2^{-j-2}, 2^{-j+2}\right]}\left|\phi(t \sqrt{L}) \psi(t \sqrt{L}) \psi_{j}(\sqrt{L}) f(x)\right|^{r} \lesssim \int_{2^{-j-2}}^{2^{-j+2}}\left|\varphi_{\lambda}^{*}(s \sqrt{L}) f(x)\right|^{r} \frac{d s}{s} .
$$

This and (44) yield (43).

\section{Besov and Triebel-Lizorkin spaces associated with $L$ : properties and characterizations}

\subsection{Definitions of Besov and Triebel-Lizorkin spaces associated with $L$}

Definition 3.1. Let $\psi$ be a partition of unity. For $0<p, q \leqslant \infty, \alpha \in \mathbb{R}$ and $w \in A_{\infty}$, we define the weighted homogeneous Besov space $\dot{B}_{p, q, w}^{\alpha, \psi, L}(X)$ as follows:

$$
\dot{B}_{p, q, w}^{\alpha, \psi, L}(X)=\left\{f \in \mathcal{S}_{\infty}^{\prime}:\|f\|_{\dot{B}_{p, q, w}^{\alpha, \psi, L}(X)}<\infty\right\}
$$

where

$$
\|f\|_{\dot{B}_{p, q, w}^{\alpha, \psi}(X)}=\left\{\sum_{j \in \mathbb{Z}}\left(2^{j \alpha}\left\|\psi_{j}(\sqrt{L}) f\right\|_{p, w}\right)^{q}\right\}^{1 / q} .
$$

Similarly, for $0<p<\infty, 0<q \leqslant \infty, \alpha \in \mathbb{R}$ and $w \in A_{\infty}$, the weighted homogeneous Triebel-Lizorkin space $\dot{F}_{p, q, w}^{\alpha, \psi, L}(X)$ is defined by

$$
\dot{F}_{p, q, w}^{\alpha, \psi}(X)=\left\{f \in \mathcal{S}_{\infty}^{\prime}:\|f\|_{\dot{F}_{p, q, w}^{\alpha, \psi, L}(X)}<\infty\right\}
$$

where

$$
\|f\|_{\dot{F}_{p, q, w}^{\alpha, \psi, L}(X)}=\left\|\left[\sum_{j \in \mathbb{Z}}\left(2^{j \alpha}\left|\psi_{j}(\sqrt{L}) f\right|\right)^{q}\right]^{1 / q}\right\|_{p, w} .
$$


We now claim that $\psi_{j}(\sqrt{L}) f=0$ for all $j \in \mathbb{Z}$ if and only if $f \in \mathscr{P}$. Indeed, since $\mathscr{P}=\bigcup_{m \in \mathbb{N}} \mathscr{P}_{m}$, it is obvious that if $f \in \mathscr{P}$, then $\psi_{j}(\sqrt{L}) f=0$ for all $j$.

For the reverse direction, we assume that $\psi_{j}(\sqrt{L}) f=0$ for all $j \in \mathbb{Z}$. Since $f \in \mathcal{S}_{\infty}^{\prime}=\mathcal{S}^{\prime} / \mathscr{P}$, we have $f=f_{\mathcal{S}^{\prime}}+f_{\mathscr{P}}$ for some $f_{\mathcal{S}^{\prime}} \in \mathcal{S}^{\prime}$ and $f_{\mathscr{P}} \in \mathscr{P}$. It follows that, for every $j \in \mathbb{Z}$,

$$
\psi_{j}(\sqrt{L}) f=\psi_{j}(\sqrt{L}) f_{\mathcal{S}^{\prime}}+\psi_{j}(\sqrt{L}) f_{\mathscr{P}}=\psi_{j}(\sqrt{L}) f_{\mathcal{S}^{\prime}} .
$$

Therefore,

$$
\sum_{j \in \mathbb{Z}} \psi_{j}(\sqrt{L}) f=\sum_{j \in \mathbb{Z}} \psi_{j}(\sqrt{L}) f_{\mathcal{S}^{\prime}}
$$

which implies

$$
\sum_{j \in \mathbb{Z}} \psi_{j}(\sqrt{L}) f_{\mathcal{S}^{\prime}}=0
$$

On the other hand, arguing similarly to the proof of Proposition 2.11, we can find $\rho \in \mathscr{P}$ such that

$$
\sum_{j \in \mathbb{Z}} \psi_{j}(\sqrt{L}) f_{\mathcal{S}^{\prime}}=f_{\mathcal{S}^{\prime}}-\rho \quad \text { in } \mathcal{S}^{\prime} .
$$

From the last two identities, we obtain $f_{\mathcal{S}^{\prime}}=\rho \in \mathscr{P}$ and hence $f \in \mathscr{P}$. The statement is proved.

Therefore, each of the above spaces is a quasinormed linear space (normed linear space when $p, q \geqslant 1$ ). Note that like the classical case, the Triebel-Lizorkin spaces for $p=\infty$ would be defined in a different way. See Section 3.5.

From Proposition 2.16, we have the following.

Proposition 3.2. Let $\psi, \varphi$ be partitions of unity and assume $\operatorname{supp} \psi, \operatorname{supp} \varphi \subset$ $[1 / 2,2]$. Let $w \in A_{\infty}, \alpha \in \mathbb{R}$ and $\lambda>0$. Then the following norm equivalence holds: for all $f \in \mathcal{S}_{\infty}^{\prime}$

$$
\begin{gathered}
\left\{\sum_{j \in \mathbb{Z}}\left(2^{j \alpha}\left\|\psi_{j, \lambda}^{*}(\sqrt{L}) f\right\|_{p, w}\right)^{q}\right\}^{1 / q} \\
\sim\left\{\sum_{j \in \mathbb{Z}}\left(2^{j \alpha}\left\|\varphi_{j, \lambda}^{*}(\sqrt{L}) f\right\|_{p, w}\right)^{q}\right\}^{1 / q}, \quad 0<p, q \leqslant \infty ; \\
\left\|\left[\sum_{j \in \mathbb{Z}}\left(2^{j \alpha}\left|\psi_{j, \lambda}^{*}(\sqrt{L}) f\right|\right)^{q}\right]^{1 / q}\right\|_{p, w} \\
\sim\left\|\left[\sum_{j \in \mathbb{Z}}\left(2^{j \alpha}\left|\varphi_{j, \lambda}^{*}(\sqrt{L}) f\right|\right)^{q}\right]^{1 / q}\right\|_{p, w}, \quad 0<p<\infty, 0<q \leqslant \infty .
\end{gathered}
$$

(b) 
We next prove the following result.

Proposition 3.3. Let $\psi$ be a partition of unity. Then we have

(a) For $0<p, q \leqslant \infty, \alpha \in \mathbb{R}$ and $\lambda>n q_{w} / p$,

$$
\left\{\sum_{j \in \mathbb{Z}}\left(2^{j \alpha}\left\|\psi_{j, \lambda}^{*}(\sqrt{L}) f\right\|_{p, w}\right)^{q}\right\}^{1 / q} \sim\|f\|_{\dot{B}_{p, q, w}^{\alpha, \psi}(X)} .
$$

(b) For $0<p<\infty, 0<q \leqslant \infty, \alpha \in \mathbb{R}$ and $\lambda>\max \left\{n / q, n q_{w} / p\right\}$,

$$
\left\|\left[\sum_{j \in \mathbb{Z}}\left(2^{j \alpha}\left|\psi_{j, \lambda}^{*}(\sqrt{L}) f\right|\right)^{q}\right]^{1 / q}\right\|_{p, w} \sim\|f\|_{\dot{F}_{p, q, w}^{\alpha, \psi}(X)} .
$$

Proof. We will provide the proof for (b) since the proof of (a) is similar and even easier. Observe that from Proposition 3.2, it suffices to prove that

$$
\left\|\left[\sum_{j \in \mathbb{Z}}\left(2^{j \alpha}\left|\psi_{j, \lambda}^{*}(\sqrt{L}) f\right|\right)^{q}\right]^{1 / q}\right\|_{p, w} \lesssim\left\|\left[\sum_{j \in \mathbb{Z}}\left(2^{j \alpha}\left|\psi_{j}(\sqrt{L}) f\right|\right)^{q}\right]^{1 / q}\right\|_{p, w} .
$$

Indeed, taking $r<\min \left\{p, q, p / q_{w}\right\}=\min \left\{q, p / q_{w}\right\}$ so that $\lambda>n / r$ and $w \in$ $A_{p / r}$, then applying (40), we have

$$
\begin{aligned}
\psi_{j, \lambda}^{*}(\sqrt{L}) f(x) & \lesssim\left[\int_{X} \frac{1}{V\left(z, 2^{-j}\right)} \frac{\left|\psi_{j}(\sqrt{L}) f(z)\right|^{r}}{\left(1+2^{j} d(x, z)\right)^{\lambda r}} d \mu(z)\right]^{1 / r} \\
& \lesssim \mathcal{M}_{r}\left(\mid \psi_{j}(\sqrt{L}) f\right)(x),
\end{aligned}
$$

where we use Lemma 2.2 in the last inequality. The desired inequality (45) then follows by using the weighted Fefferman-Stein maximal inequality (8).

As a consequence of Propositions 3.2 and 3.3, we obtain the following theorem.

THEOREM 3.4. Let $\psi$ and $\varphi$ be partitions of unity. Then the following statements hold:

(a) The spaces $\dot{B}_{p, q, w}^{\alpha, \psi, L}(X)$ and $\dot{B}_{p, q, w}^{\alpha, \varphi, L}(X)$ coincide with equivalent norms for all $0<p, q \leqslant \infty, \alpha \in \mathbb{R}$ and $w \in A_{\infty}$.

(b) The spaces $\dot{F}_{p, q, w}^{\alpha, \psi}(X)$ and $\dot{F}_{p, q, w}^{\alpha, \varphi, L}(X)$ coincide with equivalent norms for all $0<p<\infty, 0<q \leqslant \infty, \alpha \in \mathbb{R}$ and $w \in A_{\infty}$. 
For this reason, we define the spaces $\dot{B}_{p, q, w}^{\alpha, L}(X)$ and $\dot{F}_{p, q, w}^{\alpha, L}(X)$ to be any spaces $\dot{B}_{p, q, w}^{\alpha, \psi, L}(X)$ and $\dot{B}_{p, q, w}^{\alpha, \psi, L}(X)$ with any partitions of unity $\psi$, respectively.

It is routine to show that the spaces $\dot{B}_{p, q, w}^{\alpha, L}(X)$ and $\dot{F}_{p, q, w}^{\alpha, L}(X)$ are complete, and each is continuously embedded into $\mathcal{S}_{\infty}^{\prime}$. We omit the details.

3.2. Continuous characterizations by functions with compact supports. In this section, we will prove continuous characterizations for new Besov and Triebel-Lizorkin spaces including those using Lusin functions and the Littlewood-Paley functions.

THEOREM 3.5. Let $\psi$ be a partition of unity. Then we have

(a) For $w \in A_{\infty}, 0<p, q \leqslant \infty, \alpha \in \mathbb{R}$ and $\lambda>n q_{w} / p$,

$$
\begin{aligned}
\|f\|_{\dot{B}_{p, q, w}^{\alpha, L}} & \sim\left(\int_{0}^{\infty}\left[t^{-\alpha}\|\psi(t \sqrt{L}) f\|_{p, w}\right]^{q} \frac{d t}{t}\right)^{1 / q} \\
& \sim\left(\int_{0}^{\infty}\left[t^{-\alpha}\left\|\psi_{\lambda}^{*}(t \sqrt{L}) f\right\|_{p, w}\right]^{q} \frac{d t}{t}\right)^{1 / q}
\end{aligned}
$$

for all $f \in \mathcal{S}_{\infty}^{\prime}$.

(b) For $w \in A_{\infty}, 0<p<\infty, 0<q \leqslant \infty, \alpha \in \mathbb{R}$, and $\lambda>\max \left\{n / q, n q_{w} / p\right\}$,

$$
\begin{aligned}
\|f\|_{\dot{F}_{p, q, w}^{\alpha, L}} & \sim\left\|\left(\int_{0}^{\infty}\left[t^{-\alpha}|\psi(t \sqrt{L}) f|\right]^{q} \frac{d t}{t}\right)\right\|_{p, w} \\
& \sim\left\|\left(\int_{0}^{\infty}\left[t^{-\alpha} \psi_{\lambda}^{*}(t \sqrt{L}) f\right]^{q} \frac{d t}{t}\right)\right\|_{p, w}
\end{aligned}
$$

for all $f \in \mathcal{S}_{\infty}^{\prime}$.

Proof. We give the proof of (b) only since the proof of (a) can be done in the same manner.

We divide the proof of (b) into three steps.

Step 1: We first prove that

$$
\left\|\left(\int_{0}^{\infty}\left[t^{-\alpha}|\psi(t \sqrt{L}) f|\right]^{q} \frac{d t}{t}\right)^{1 / q}\right\|_{p, w} \lesssim\|f\|_{\dot{F}_{p, q, w}^{\alpha, L}} .
$$


Indeed, for $t \in\left[2^{-j-1}, 2^{-j}\right]$ with $j \in \mathbb{Z}$, from (36), we see that

$$
\sup _{t \in\left[2^{-j-1}, 2^{-j}\right]}|\psi(t \sqrt{L}) f(x)| \lesssim \sum_{k=j-2}^{j+3} \psi_{k, \lambda}^{*}(\sqrt{L}) f(x) .
$$

Hence, the estimate (48) follows from the above inequality and Proposition 3.2.

Step 2: We next show that

$$
\|f\|_{\dot{F}_{p, q, w}^{\alpha, L}} \lesssim\left\|\left(\int_{0}^{\infty}\left[t^{-\alpha} \psi_{\lambda}^{*}(t \sqrt{L}) f\right]^{q} \frac{d t}{t}\right)^{1 / q}\right\|_{p, w}
$$

Using Proposition 2.18, we have

$$
\left|\psi_{j}(\sqrt{L}) f(x)\right| \lesssim\left(\int_{2^{-j-2}}^{2^{-j+2}}\left|\psi_{\lambda}^{*}(s \sqrt{L}) f(x)\right|^{q} \frac{d s}{s}\right)^{1 / q} .
$$

This implies the desired inequality (49).

Step 3: To complete the proof of the theorem, we need to prove that

$$
\left\|\left(\int_{0}^{\infty}\left[t^{-\alpha} \psi_{\lambda}^{*}(t \sqrt{L}) f\right]^{q} \frac{d t}{t}\right)^{1 / q}\right\|_{p, w} \lesssim\left\|\left(\int_{0}^{\infty}\left[t^{-\alpha}|\psi(t \sqrt{L}) f|\right]^{q} \frac{d t}{t}\right)^{1 / q}\right\|_{p, w} .
$$

To see this, taking $r<\min \left\{p, q, p / q_{w}\right\}=\min \left\{q, p / q_{w}\right\}$ so that $\lambda>n / r$ and $w \in A_{p / r}$, then applying (40), we have, for all $t \in[1,2]$,

$$
\left|\psi_{\lambda}^{*}\left(2^{-j} t \sqrt{L}\right) f(x)\right|^{r} \lesssim \int_{X} \frac{1}{V\left(x, 2^{-j}\right)} \frac{\left|\psi\left(2^{-j} t \sqrt{L}\right) f(z)\right|^{r}}{\left(1+2^{j} d(x, z)\right)^{\lambda r}} d \mu(z) .
$$

Since $r<q$, we use Minkowski's inequality to get the bound:

$$
\begin{aligned}
& \left(\int_{1}^{2}\left|\psi_{\lambda}^{*}\left(2^{-j} t \sqrt{L}\right) f(x)\right|^{q} \frac{d t}{t}\right)^{r / q} \\
& \quad \lesssim \int_{X} \frac{1}{V\left(z, 2^{-j}\right)} \frac{\left(\int_{1}^{2}\left|\psi\left(2^{-j} t \sqrt{L}\right) f(z)\right|^{q} \frac{d t}{t}\right)^{r / q}}{\left(1+2^{j} d(x, z)\right)^{\lambda r}} d \mu(z) .
\end{aligned}
$$

By a change of variables,

$$
\left[\int_{2^{-j}}^{2^{-j+1}}\left(t^{-\alpha}\left|\psi_{\lambda}^{*}(t \sqrt{L}) f(x)\right|\right)^{q} \frac{d t}{t}\right]^{r / q}
$$




$$
\lesssim \int_{X} \frac{1}{V\left(z, 2^{-j}\right)} \frac{\left[\int_{2^{-j}}^{2^{-j+1}}\left(t^{-\alpha}|\psi(t \sqrt{L}) f(z)|\right)^{q} \frac{d t}{t}\right]^{r / q}}{\left(1+2^{j} d(x, z)\right)^{\lambda r}} d \mu(z)
$$

Hence, applying Lemma 2.2, we obtain

$$
\left(\int_{2^{-j}}^{2^{-j+1}}\left|\psi_{\lambda}^{*}(t \sqrt{L}) f(x)\right|^{q} \frac{d t}{t}\right)^{1 / q} \lesssim \mathcal{M}_{r}\left[\left(\int_{2^{-j}}^{2^{-j+1}}|\psi(t \sqrt{L}) f|^{q} \frac{d t}{t}\right)^{1 / q}\right](x)
$$

as long as $\lambda r>n$. Using (8), we deduced the required estimate (50).

The proof of our theorem is thus complete.

3.3. Continuous characterizations by functions in $\mathscr{S}_{m}(\mathbb{R})$. For each $m \in \mathbb{N}$, we denote by $\mathscr{S}_{m}(\mathbb{R})$ the set of all even functions $\varphi \in \mathscr{S}(\mathbb{R})$ such that $\varphi(\xi)=$ $\xi^{2 m} \phi(\xi)$ for some $\phi \in \mathscr{S}(\mathbb{R})$ and $\varphi(\xi) \neq 0$ on $(-2,-1 / 2) \cup(1 / 2,2)$.

We have the following characterization for the new Besov and Triebel-Lizorkin spaces via functions in $\mathscr{S}_{m}(\mathbb{R})$.

TheOREM 3.6. Let $w \in A_{\infty}, \alpha \in \mathbb{R}, m>\alpha / 2$ and let $\varphi \in \mathscr{S}_{m}(\mathbb{R})$. Then the following statements hold:

(a) For $0<p, q \leqslant \infty, \lambda>n q_{w} / p$ and $f \in \mathcal{S}^{\prime}$, there exists $\rho \in \mathscr{P}$ so that

$$
\begin{aligned}
& \left(\int_{0}^{\infty}\left[t^{-\alpha}\left\|\varphi_{\lambda}^{*}(t \sqrt{L})(f-\rho)\right\|_{p, w}\right]^{q} \frac{d t}{t}\right)^{1 / q} \lesssim\|f\|_{\dot{B}_{p, q, w}^{\alpha, L}} \\
& \quad \lesssim\left(\int_{0}^{\infty}\left[t^{-\alpha}\|\varphi(t \sqrt{L}) f\|_{p, w}\right]^{q} \frac{d t}{t}\right)^{1 / q} .
\end{aligned}
$$

(b) For $0<p<\infty, 0<q \leqslant \infty, \lambda>\max \left\{n / q, n q_{w} / p\right\}$ and $f \in \mathcal{S}^{\prime}$, there exists $\rho \in \mathscr{P}$ so that

$$
\begin{aligned}
& \left\|\left(\int_{0}^{\infty}\left[t^{-\alpha} \varphi_{\lambda}^{*}(t \sqrt{L})(f-\rho)\right]^{q} \frac{d t}{t}\right)^{1 / q}\right\|_{p, w} \lesssim\|f\|_{F_{p, q, w}^{\alpha, L}} \\
& \quad \lesssim\left\|\left(\int_{0}^{\infty}\left[t^{-\alpha} \varphi(t \sqrt{L}) f\right]^{q} \frac{d t}{t}\right)^{1 / q}\right\|_{p, w} .
\end{aligned}
$$

Proof. In comparison with the proof of Theorem 3.5, the proof of Theorem 3.6 is much more difficult due to the lack of compact support condition for the functions in $\mathscr{S}_{m}(\mathbb{R})$. A significant difference with Theorem 3.5 is that the results are formulated for $f \in \mathcal{S}^{\prime}$ (as opposed to $f \in \mathcal{S}_{\infty}^{\prime}$ ). The reason being that, 
as $K_{\varphi(t \sqrt{L})}(x, \cdot)$ may not be in $\mathcal{S}_{\infty}$ for $\varphi \in \mathscr{S}_{m}(\mathbb{R}), \varphi(t \sqrt{L}) f$ may not be defined when $f \in \mathcal{S}_{\infty}^{\prime}$. Although each $f \in \mathcal{S}_{\infty}^{\prime}$ has an extension to an element in $\mathcal{S}^{\prime}$, the extension is not unique; that is, $\varphi(t \sqrt{L}) f$ will depend on the chosen representative of $f$. Our theorem says that there exists a representative so that the left-hand-side inequality in (51) (or in (52)) holds.

We will prove (52) only because the proof of (51) can be done in the same manner. We divide the proof into a number of steps.

Step 1: Let $\psi$ be a partition of unity. From Proposition 2.11, there exists $\rho \in \mathscr{P}$ so that

$$
f-\rho=c_{\psi} \int_{0}^{\infty} \psi(s \sqrt{L}) f \frac{d s}{s} \quad \text { in } \mathcal{S}^{\prime} .
$$

We will show that

$$
\left\|\left(\int_{0}^{\infty}\left[t^{-\alpha} \varphi_{\lambda}^{*}(t \sqrt{L})(f-\rho)\right]^{q} \frac{d t}{t}\right)^{1 / q}\right\|_{p, w} \lesssim\|f\|_{\dot{F}_{p, q, w}^{\alpha, L}} .
$$

First, note that the Calderón reproducing formula above implies the pointwise representation

$$
\varphi(t \sqrt{L})(f-\rho)=c_{\psi} \int_{0}^{\infty} \varphi(t \sqrt{L}) \psi(s \sqrt{L}) f \frac{d s}{s}
$$

for all $t>0$.

Let $\lambda>0, t \in\left[2^{-v-1}, 2^{-v}\right]$ for some $v \in \mathbb{Z}$ and $M>m+\lambda / 2$. For simplicity of writing, we let $c_{\psi}=1$. We then have

$$
\begin{aligned}
\varphi( & t \sqrt{L})(f-\rho)=\int_{0}^{\infty} \psi(s \sqrt{L}) \varphi(t \sqrt{L}) f \frac{d s}{s} \\
= & \sum_{j \geqslant v} \int_{2^{-j-1}}^{2^{-j}} \psi(s \sqrt{L}) \varphi(t \sqrt{L}) f \frac{d s}{s}+\sum_{j<v} \int_{2^{-j-1}}^{2^{-j}} \psi(s \sqrt{L}) \varphi(t \sqrt{L}) f \frac{d s}{s} \\
= & \sum_{j \geqslant v} \int_{2^{-j-1}}^{2^{-j}}\left(\frac{s}{t}\right)^{2 M}\left(s^{2} L\right)^{-M} \psi(s \sqrt{L})\left(t^{2} L\right)^{M} \varphi(t \sqrt{L}) f \frac{d s}{s} \\
& +\sum_{j<v} \int_{2^{-j-1}}^{2^{-j}}\left(\frac{t}{s}\right)^{2 m}\left(s^{2} L\right)^{m} \psi(s \sqrt{L})\left(t^{2} L\right)^{-m} \varphi(t \sqrt{L}) f \frac{d s}{s} .
\end{aligned}
$$

Setting $\psi_{s, M}(x)=(s x)^{-2 M} \psi(s x)$ and $\tilde{\psi}_{s, m}(x)=(s x)^{2 m} \psi(s x)$, we rewrite the 
above as

$$
\begin{aligned}
\varphi(t \sqrt{L})(f-\rho)= & \sum_{j \geqslant v} \int_{2^{-j-1}}^{2^{-j}}\left(\frac{s}{t}\right)^{2 M}\left(t^{2} L\right)^{M} \varphi(t \sqrt{L}) \psi_{s, M}(\sqrt{L}) f \frac{d s}{s} \\
& +\sum_{j<v} \int_{2^{-j-1}}^{2^{-j}}\left(\frac{t}{s}\right)^{2 m}\left(t^{2} L\right)^{-m} \varphi(t \sqrt{L}) \tilde{\psi}_{s, m}(\sqrt{L}) f \frac{d s}{s} .
\end{aligned}
$$

Since $\xi^{2 M} \varphi(\xi)$ is an even function in $\mathscr{S}(\mathbb{R})$, we use Lemma 2.6 to deduce that

$$
\begin{aligned}
& \left|\left(t^{2} L\right)^{M} \varphi(t \sqrt{L}) \psi_{s, N}(\sqrt{L}) f(y)\right| \\
& \quad \lesssim \int_{X} \frac{1}{V(y, t)}\left(1+\frac{d(y, z)}{t}\right)^{-\lambda-N}\left|\psi_{s, N}(\sqrt{L}) f(z)\right| d \mu(z),
\end{aligned}
$$

where $N>n$.

It follows that

$$
\begin{aligned}
& \frac{\left|\left(t^{2} L\right)^{M} \varphi(t \sqrt{L}) \psi_{s, N}(\sqrt{L}) f(y)\right|}{(1+d(x, y) / t)^{\lambda}} \\
& \quad \lesssim \int_{X} \frac{1}{V(y, t)}\left(1+\frac{d(y, z)}{t}\right)^{-N} \frac{\left|\psi_{s, N}(\sqrt{L}) f(z)\right|}{(1+d(x, z) / t)^{\lambda}} d \mu(z)
\end{aligned}
$$

for all $x, y \in X$.

Hence, for $j \geqslant v, t \in\left[2^{-v-1}, 2^{-v}\right]$ and $s \in\left[2^{-j-1}, 2^{-j}\right]$, we have

$$
\begin{aligned}
& \frac{\left|\left(t^{2} L\right)^{M} \varphi(t \sqrt{L}) \psi_{s, N}(\sqrt{L}) f(y)\right|}{(1+d(x, y) / t)^{\lambda}} \\
& \quad \lesssim 2^{\lambda(j-v)} \psi_{s, N, \lambda}^{*}(\sqrt{L}) f(x) \int_{X} \frac{1}{V(y, t)}\left(1+\frac{d(y, z)}{t}\right)^{-N} d \mu(y) \\
& \quad \lesssim 2^{\lambda(j-v)} \psi_{s, N, \lambda}^{*}(\sqrt{L}) f(x) .
\end{aligned}
$$

Since $\psi \in \mathscr{S}_{m}(\mathbb{R}), x^{-2 m} \psi(x) \in \mathscr{S}(\mathbb{R})$. Using Lemma 2.6 and an argument similar to the above estimate for $\psi_{s, M}$, we obtain, for $j<v, t \in\left[2^{-v-1}, 2^{-v}\right]$ and $s \in\left[2^{-j-1}, 2^{-j}\right]$,

$$
\frac{\left|\left(t^{2} L\right)^{-m} \varphi(t \sqrt{L}) \tilde{\psi}_{s, m}(\sqrt{L}) f(y)\right|}{(1+d(x, y) / t)^{\lambda}} \lesssim \tilde{\psi}_{s, m, \lambda}^{*}(\sqrt{L}) f(x) .
$$

Combining the above two estimates, we deduce that

$$
\begin{aligned}
\left|\varphi_{\lambda}^{*}(t \sqrt{L})(f-\rho)\right| \leqslant & \sum_{j \geqslant v} 2^{-(j-v)(2 M-\lambda)} \sup _{s \in\left(2^{-j-1}, 2^{-j}\right]} \psi_{s, N, \lambda}^{*}(\sqrt{L}) f \\
& +\sum_{j<v} 2^{-2 m(v-j)} \sup _{s \in\left(2^{-j-1}, 2^{-j}\right]} \tilde{\psi}_{s, m, \lambda}^{*}(\sqrt{L}) f .
\end{aligned}
$$


This, along with Proposition 2.16, implies that

$$
\begin{aligned}
\left|\varphi_{\lambda}^{*}(t \sqrt{L})(f-\rho)\right| & \lesssim \sum_{j \geqslant v-1} 2^{-(2 M-\lambda)(j-v)} \psi_{j, \lambda}^{*}(\sqrt{L}) f+\sum_{j<v+3} 2^{-2 m(v-j)} \psi_{j, \lambda}^{*}(\sqrt{L}) f \\
& \lesssim \sum_{j \in \mathbb{Z}} 2^{-2 m|v-j|} \psi_{j, \lambda}^{*}(\sqrt{L}) f
\end{aligned}
$$

for all $t \in\left[2^{-v-1}, 2^{-v}\right]$ and $M>m+\lambda / 2$. Therefore, if $q \leqslant 1$, we have

$$
\int_{2^{-v-1}}^{2^{-v}}\left(t^{-\alpha}\left|\varphi_{\lambda}^{*}(t \sqrt{L})(f-\rho)\right|\right)^{q} \frac{d t}{t} \lesssim \sum_{j \in \mathbb{Z}} 2^{-q(2 m-\alpha)|v-j|}\left(2^{j \alpha} \psi_{j, \lambda}^{*}(\sqrt{L}) f\right)^{q} .
$$

It follows that

$$
\begin{aligned}
& \left(\int_{0}^{\infty}\left(t^{-\alpha}\left|\varphi_{\lambda}^{*}(t \sqrt{L})(f-\rho)\right|\right)^{q} \frac{d t}{t}\right)^{1 / q} \\
& \quad \lesssim\left(\sum_{\nu \in \mathbb{Z}} \sum_{j \in \mathbb{Z}} 2^{-q(2 m-\alpha)|\nu-j|}\left(2^{j \alpha} \psi_{j, \lambda}^{*}(\sqrt{L}) f\right)^{q}\right)^{1 / q} \\
& \quad \lesssim\left(\sum_{j \in \mathbb{Z}}\left(2^{j \alpha} \psi_{j, \lambda}^{*}(\sqrt{L}) f\right)^{q}\right)^{1 / q},
\end{aligned}
$$

which, along with Proposition 3.3, yields (53).

On the other hand, if $q>1$, then we use Young's inequality to get the bound:

$$
\begin{aligned}
& \left(\int_{0}^{\infty}\left(t^{-\alpha}\left|\varphi_{\lambda}^{*}(t \sqrt{L})(f-\rho)\right|\right)^{q} \frac{d t}{t}\right)^{1 / q} \\
& \quad \lesssim\left(\sum_{\nu \in \mathbb{Z}}\left[\sum_{j \in \mathbb{Z}} 2^{-(2 m-\alpha)|\nu-j|} 2^{j \alpha} \psi_{j, \lambda}^{*}(\sqrt{L}) f\right]^{q}\right)^{1 / q} \\
& \quad \lesssim\left(\sum_{j \in \mathbb{Z}}\left(2^{j \alpha} \psi_{j, \lambda}^{*}(\sqrt{L}) f\right)^{q}\right)^{1 / q} .
\end{aligned}
$$

Hence, (53) follows from this and Proposition 3.3.

Step 2: We next show that

$$
\|f\|_{\dot{F}_{p, q, w}^{\alpha, L}} \lesssim\left\|\left(\int_{0}^{\infty}\left[t^{-\alpha} \varphi_{\lambda}^{*}(t \sqrt{L}) f\right]^{q} \frac{d t}{t}\right)^{1 / q}\right\|_{p, w}
$$


Let $\psi$ be a partition of unity. By Proposition 2.18,

$$
\left|\psi_{j}(\sqrt{L}) f(x)\right|^{q} \lesssim \int_{2^{-j-2}}^{2^{-j+2}}\left|\varphi_{\lambda}^{*}(t \sqrt{L}) f(x)\right|^{q} \frac{d s}{s} .
$$

Hence, the desired inequality follows.

Step 3: This is the most elaborate step in the proof, where we will prove that

$$
\left\|\left(\int_{0}^{\infty}\left[t^{-\alpha} \varphi_{\lambda}^{*}(t \sqrt{L}) f\right]^{q} \frac{d t}{t}\right)^{1 / q}\right\|_{p, w} \lesssim\left\|\left(\int_{0}^{\infty}\left[t^{-\alpha} \varphi(t \sqrt{L}) f\right]^{q} \frac{d t}{t}\right)^{1 / q}\right\|_{p, w} .
$$

To this end, we apply Lemma 2.12 to find $a, b, c>0$ and even functions $\phi$, $\eta \in \mathscr{S}(\mathbb{R})$ with supp $\phi \subset[-a, a]$, supp $\eta \subset[-c,-b] \cup[b, c]$, and so that for every $\ell \in \mathbb{Z}, t \in[1,2]$ and $f \in \mathcal{S}^{\prime}$, we have

$$
f=\phi\left(2^{-\ell} t \sqrt{L}\right) f+\sum_{k \geqslant 1} \varphi\left(2^{-k-\ell} t \sqrt{L}\right) \eta\left(2^{-k-\ell} t \sqrt{L}\right) f \quad \text { in } \mathcal{S}^{\prime} .
$$

This implies that

$$
\begin{aligned}
& \varphi\left(2^{-\ell} t \sqrt{L}\right) f \\
& \quad=\phi\left(2^{-\ell} t \sqrt{L}\right) \varphi\left(2^{-\ell} t \sqrt{L}\right) f+\sum_{k \geqslant 1} \varphi\left(2^{-k-\ell} t \sqrt{L}\right) \eta\left(2^{-k-\ell} t \sqrt{L}\right) \varphi\left(2^{-\ell} t \sqrt{L}\right) f
\end{aligned}
$$

pointwise.

Let $\lambda, A>0$. Put $M=\lambda+A$. Using the above pointwise representation together with Lemma 2.6, we get

$$
\begin{aligned}
& \left|\varphi\left(2^{-\ell} t \sqrt{L}\right) f(y)\right| \lesssim \int_{X} \frac{1}{V\left(z, 2^{-\ell}\right)}\left(1+2^{\ell} d(y, z)\right)^{-\lambda}\left|\varphi\left(2^{-\ell} t \sqrt{L}\right) f(z)\right| d \mu(z) \\
& \quad+\sum_{k \geqslant 1} 2^{-k M} \int_{X} \frac{1}{V\left(z, 2^{-\ell}\right)}\left(1+2^{\ell} d(y, z)\right)^{-\lambda}\left|\varphi\left(2^{-\ell-k} t \sqrt{L}\right) f(z)\right| d \mu(z) \\
& =\sum_{k \geqslant 0} 2^{-k M} \int_{X} \frac{1}{V\left(z, 2^{-\ell}\right)}\left(1+2^{\ell} d(y, z)\right)^{-\lambda}\left|\varphi\left(2^{-\ell-k} t \sqrt{L}\right) f(z)\right| d \mu(z) \\
& =2^{(\ell-j) M} \sum_{k \geqslant \ell} 2^{(j-k) M} \int_{X} \frac{1}{V\left(z, 2^{-\ell}\right)}\left(1+2^{\ell} d(y, z)\right)^{-\lambda}\left|\varphi\left(2^{-k} t \sqrt{L}\right) f(z)\right| d \mu(z) \\
& \leqslant 2^{(\ell-j) M} \sum_{k \geqslant \ell} 2^{(j-k) M} \int_{X} \frac{1}{V\left(z, 2^{-k}\right)} \frac{\left|\varphi\left(2^{-k} t \sqrt{L}\right) f(z)\right|}{\left(1+2^{\ell} d(y, z)\right)^{\lambda}} d \mu(z),
\end{aligned}
$$


where $j, \ell \in \mathbb{Z}$ and $\ell \geqslant j$. It follows that, for any $0<r \leqslant 1$,

$$
\begin{aligned}
& 2^{(j-\ell) M} \frac{\left|\varphi\left(2^{-\ell} t \sqrt{L}\right) f(y)\right|}{\left(1+2^{j} d(x, y)\right)^{\lambda}} \\
& \vdots \sum_{k \geqslant \ell} 2^{(j-k) M} \int_{X} \frac{1}{V\left(z, 2^{-k}\right)} \frac{\left|\varphi\left(2^{-k} t \sqrt{L}\right) f(z)\right|}{\left(1+2^{\ell} d(y, z)\right)^{\lambda}\left(1+2^{j} d(x, y)\right)^{\lambda}} d \mu(z) \\
& \leqslant \sum_{k \geqslant \ell} 2^{(j-k) M} \int_{X} \frac{1}{V\left(z, 2^{-k}\right)} \frac{\left|\varphi\left(2^{-k} t \sqrt{L}\right) f(z)\right|}{\left(1+2^{j} d(y, z)\right)^{\lambda}\left(1+2^{j} d(x, y)\right)^{\lambda}} d \mu(z) \\
& \leqslant \sum_{k \geqslant \ell} 2^{(j-k) M} \int_{X} \frac{1}{V\left(z, 2^{-k}\right)} \frac{\left|\varphi\left(2^{-k} t \sqrt{L}\right) f(z)\right|}{\left(1+2^{j} d(x, z)\right)^{\lambda}} d \mu(z) \\
& \leqslant \varphi_{A, \lambda}^{* *}\left(2^{-j} t \sqrt{L}\right) f(x)^{1-r} \\
& \quad \times \sum_{k \geqslant j} 2^{(j-k) M r} \int_{X} \frac{1}{V\left(z, 2^{-k}\right)} \frac{\left|\varphi\left(2^{-k} t \sqrt{L}\right) f(z)\right|^{r}}{\left(1+2^{j} d(x, z)\right)^{\lambda r}} d \mu(z)
\end{aligned}
$$

where, for each $j \in \mathbb{Z}$ and $t \in[1,2]$, we define the Peetre-type maximal function by

$$
\varphi_{A, \lambda}^{* *}\left(2^{-j} t \sqrt{L}\right) f(x)=\sup _{k \geqslant j} \sup _{y \in X} 2^{(j-k) M} \frac{\left|\varphi\left(2^{-k} t \sqrt{L}\right) f(y)\right|}{\left(1+2^{j} d(x, y)\right)^{\lambda}} .
$$

By (19), $\varphi_{A, \lambda}^{* *}\left(2^{-j} t \sqrt{L}\right) f(x)<\infty$, for all sufficiently large $\lambda$ (depending on $f$ ), all $x \in X$ and $A>0$. Hence, for any such $\lambda$, by taking the supremum of the LHS of (56) over $\ell \geqslant j$ and $y \in X$ and using the obvious inequality $\left(1+2^{j} d(x\right.$, $z)) \geqslant 2^{j-k}\left(1+2^{k} d(x, z)\right)$ on the RHS, we obtain

$$
\begin{aligned}
\varphi_{A, \lambda}^{* *}\left(2^{-j} t \sqrt{L}\right) f(x)^{r} \\
\quad \lesssim \sum_{k \geqslant j} 2^{(j-k)(M-\lambda) r} \int_{X} \frac{1}{V\left(z, 2^{-k}\right)} \frac{\left|\varphi\left(2^{-k} t \sqrt{L}\right) f(z)\right|^{r}}{\left(1+2^{k} d(x, z)\right)^{\lambda r}} d \mu(z) \\
\quad=\sum_{k \geqslant j} 2^{(j-k) A r} \int_{X} \frac{1}{V\left(z, 2^{-k}\right)} \frac{\left|\varphi\left(2^{-k} t \sqrt{L}\right) f(z)\right|^{r}}{\left(1+2^{k} d(x, z)\right)^{\lambda r}} d \mu(z) \quad(\text { as } M-\lambda=A) .
\end{aligned}
$$

Since clearly $|\varphi(\cdots) f| \leqslant \varphi_{A, \lambda}^{* *}(\cdots) f$, the above implies that

$$
\left|\varphi\left(2^{-\ell} t \sqrt{L}\right) f(y)\right|^{r} \lesssim \sum_{k \geqslant \ell} 2^{(\ell-k) A r} \int_{X} \frac{1}{V\left(z, 2^{-k}\right)} \frac{\left|\varphi\left(2^{-k} t \sqrt{L}\right) f(z)\right|^{r}}{\left(1+2^{k} d(x, z)\right)^{\lambda r}} d \mu(z)
$$


for all sufficiently large $\lambda, A>0, y \in X$ and $\ell \in \mathbb{Z}$. But the right-hand side of the above inequality increases as $\lambda$ decreases, and hence this inequality holds for all $\lambda>0$ and $A>0$, with the inequality constant also depending on $f$. It follows that, for $\ell \geqslant j$,

$$
\begin{aligned}
& 2^{(j-\ell) M r} \frac{\left|\varphi\left(2^{-\ell} t \sqrt{L}\right) f(y)\right|^{r}}{\left(1+2^{j} d(x, y)\right)^{\lambda r}} \\
& \quad \lesssim \sum_{k \geqslant \ell} 2^{(j-k) A r} \int_{X} \frac{2^{(j-\ell) \lambda r}}{V\left(z, 2^{-k}\right)} \frac{\left|\varphi\left(2^{-k} t \sqrt{L}\right) f(z)\right|^{r}}{\left(1+2^{k} d(y, z)\right)^{\lambda r}\left(1+2^{j} d(x, y)\right)^{\lambda r}} d \mu(z) \\
& \quad=\sum_{k \geqslant \ell} 2^{(j-k) A r} \int_{X} \frac{2^{(k-\ell) \lambda r}}{V\left(z, 2^{-k}\right)} \frac{\left|\varphi\left(2^{-k} t \sqrt{L}\right) f(z)\right|^{r}}{\left(1+2^{k} d(y, z)\right)^{\lambda r}\left(2^{k-j}+2^{k} d(x, y)\right)^{\lambda r}} d \mu(z) \\
& \quad \leqslant \sum_{k \geqslant j} 2^{(j-k)(A-\lambda) r} \int_{X} \frac{1}{V\left(z, 2^{-k}\right)} \frac{\left|\varphi\left(2^{-k} t \sqrt{L}\right) f(z)\right|^{r}}{\left(1+2^{k} d(x, z)\right)^{\lambda r}} d \mu(z) .
\end{aligned}
$$

(Recall that $M=\lambda+A$.) Taking the supremum over $\ell \geqslant j$ and $y \in X$ gives

$$
\varphi_{A, \lambda}^{* *}\left(2^{-j} t \sqrt{L}\right) f(x)^{r} \lesssim \sum_{k \geqslant j} 2^{(j-k)(A-\lambda) r} \int_{X} \frac{1}{V\left(z, 2^{-k}\right)} \frac{\left|\varphi\left(2^{-k} t \sqrt{L}\right) f(z)\right|^{r}}{\left(1+2^{k} d(x, z)\right)^{\lambda r}} d \mu(z) .
$$

Therefore, if the right-hand side of the above is finite, then $\varphi_{A, \lambda}^{* *}\left(2^{-j} t \sqrt{L}\right) f(x)<$ $\infty$. Repeating the proof of (57), we obtain

$$
\begin{aligned}
\varphi_{A, \lambda}^{* *}\left(2^{-j} t \sqrt{L}\right) f(x)^{r} & \lesssim \sum_{k \geqslant j} 2^{(j-k) A r} \int_{X} \frac{1}{V\left(z, 2^{-k}\right)} \frac{\left|\varphi\left(2^{-k} t \sqrt{L}\right) f(z)\right|^{r}}{\left(1+2^{k} d(x, z)\right)^{\lambda r}} d \mu(z) \\
& \lesssim \sum_{k \geqslant j} 2^{(j-k)(A-\lambda) r} \int_{X} \frac{1}{V\left(z, 2^{-k}\right)} \frac{\left|\varphi\left(2^{-k} t \sqrt{L}\right) f(z)\right|^{r}}{\left(1+2^{k} d(x, z)\right)^{\lambda r}} d \mu(z)
\end{aligned}
$$

(with inequality constant independent of $f$ ). Since clearly $\varphi_{\lambda}^{*} \lesssim \varphi_{A, \lambda}^{* *}$, it follows that

$$
\varphi_{\lambda}^{*}\left(2^{-j} t \sqrt{L}\right) f(x)^{r} \lesssim \sum_{k \geqslant j} 2^{(j-k)(A-\lambda) r} \int_{X} \frac{1}{V\left(z, 2^{-k}\right)} \frac{\left|\varphi\left(2^{-k} t \sqrt{L}\right) f(z)\right|^{r}}{\left(1+2^{k} d(x, z)\right)^{\lambda r}} d \mu(z),
$$

provided the sum in the right-hand side is finite. Since (58) is obviously true when this sum is infinite, we conclude that it holds for all $\lambda>0, A>0, j \in \mathbb{Z}, t \in[1,2]$ and $0<r \leqslant 1$. 
Assume now that $r>1$. Let $\lambda>0, A>0$ and $N>n$. Using the Calderón reproducing formula and Lemma 2.6 as at the beginning of this step, we see that, for any $j \in \mathbb{Z}, t \in[1,2]$ and $y \in X$,

$$
\begin{aligned}
\left|\varphi\left(2^{-j} t \sqrt{L}\right) f(y)\right| & \lesssim \sum_{k \geqslant j} 2^{(j-k) A} \int_{X} \frac{\left(1+2^{j} d(y, z)\right)^{-N}}{V\left(z, 2^{-j}\right)} \frac{\left|\varphi\left(2^{-k} t \sqrt{L}\right) f(z)\right|}{\left(1+2^{j} d(y, z)\right)^{\lambda}} d \mu(z) \\
& \lesssim\left(\sum_{k \geqslant j} 2^{(j-k) A r} \int_{X} \frac{1}{V\left(z, 2^{-j}\right)} \frac{\left|\varphi\left(2^{-k} t \sqrt{L}\right) f(z)\right|^{r}}{\left(1+2^{j} d(y, z)\right)^{\lambda r}} d \mu(z)\right)^{1 / r} \\
& \leqslant\left(\sum_{k \geqslant j} 2^{(j-k) A r} \int_{X} \frac{1}{V\left(z, 2^{-k}\right)} \frac{\left|\varphi\left(2^{-k} t \sqrt{L}\right) f(z)\right|^{r}}{\left(1+2^{j} d(y, z)\right)^{\lambda r}} d \mu(z)\right)^{1 / r}
\end{aligned}
$$

where we have also used Lemma 2.2 and Hölder's inequality in the second last inequality in the above. It follows that

$$
\begin{aligned}
& \frac{\left|\varphi\left(2^{-j} t \sqrt{L}\right) f(y)\right|^{r}}{\left(1+2^{j} d(x, y) / t\right)^{\lambda r}} \\
& \quad \lesssim \sum_{k \geqslant j} 2^{(j-k) A r} \int_{X} \frac{1}{V\left(z, 2^{-k}\right)} \frac{\left|\varphi\left(2^{-k} t \sqrt{L}\right) f(z)\right|^{r}}{\left(1+2^{j} d(y, z)\right)^{\lambda r}\left(1+2^{j} d(x, y)\right)^{\lambda r}} d \mu(z) \\
& \quad \lesssim \sum_{k \geqslant j} 2^{(j-k) A r} \int_{X} \frac{1}{V\left(z, 2^{-k}\right)} \frac{\left|\varphi\left(2^{-k} t \sqrt{L}\right) f(z)\right|^{r}}{\left(1+2^{j} d(x, z)\right)^{\lambda r}} d \mu(z) \\
& \quad \lesssim \sum_{k \geqslant j} 2^{(j-k)(A-\lambda) r} \int_{X} \frac{1}{V\left(z, 2^{-k}\right)} \frac{\left|\varphi\left(2^{-k} t \sqrt{L}\right) f(z)\right|^{r}}{\left(1+2^{k} d(x, z)\right)^{\lambda r}} d \mu(z)
\end{aligned}
$$

Taking the supremum of the LHS over all $y \in X$, we deduce that (58) also holds for $r>1$. Hence, (58) holds for all $r>0$. Consequently,

$$
\begin{aligned}
& {\left[\left(2^{-j} t\right)^{-\alpha} \varphi_{\lambda}^{*}\left(2^{-j} t \sqrt{L}\right) f(x)\right]^{r}} \\
& \quad \lesssim \sum_{k \geqslant j} 2^{(j-k)(A-\lambda+\alpha) r} \int_{X} \frac{1}{V\left(z, 2^{-k}\right)} \frac{\left[\left(2^{-k} t\right)^{-\alpha}\left|\varphi\left(2^{-k} t \sqrt{L}\right) f(z)\right|\right]^{r}}{\left(1+2^{k} d(x, z)\right)^{\lambda r}} d \mu(z) .
\end{aligned}
$$

We now choose $r>0$ such that $\max \left\{n / p, n / q, n q_{w} / p\right\}=\max \left\{n / q, n q_{w} / p\right\}<$ $n / r<\lambda$ and then choose $A>0$ such that $A-\lambda+\alpha>0$. Minkowski's inequality and the above inequality then imply that

$$
\left(\int_{1}^{2}\left[\left(2^{-j} t\right)^{-\alpha} \varphi_{\lambda}^{*}\left(2^{-j} t \sqrt{L}\right) f(x)\right]^{q} \frac{d t}{t}\right)^{r / q}
$$




$$
\lesssim \sum_{k \geqslant j} \int_{X} \frac{2^{(j-k)(A-\lambda+\alpha) r}}{V\left(z, 2^{-k}\right)} \frac{\left(\int_{1}^{2}\left[\left(2^{-k} t\right)^{-\alpha}\left|\varphi\left(2^{-k} t \sqrt{L}\right) f(z)\right|\right]^{q} \frac{d t}{t}\right)^{r / q}}{\left(1+2^{k} d(x, z)\right)^{\lambda r}} d \mu(z)
$$

By a change of variables, we get that

$$
\begin{aligned}
& {\left[\int_{2^{-j}}^{2^{-j+1}}\left(t^{-\alpha}\left|\varphi_{\lambda}^{*}(t \sqrt{L}) f(x)\right|\right)^{q} \frac{d t}{t}\right]^{r / q}} \\
& \quad \lesssim \sum_{k \geqslant j} \int_{X} \frac{2^{(j-k)(A-\lambda+\alpha) r}}{V\left(z, 2^{-k}\right)} \frac{\left.\left[\int_{2^{-k}}^{2^{-k+1}}\left(t^{-\alpha} \mid \varphi(t \sqrt{L})\right) f(z) \mid\right)^{q} \frac{d t}{t}\right]^{r / q}}{\left(1+2^{k} d(x, z)\right)^{\lambda r}} d \mu(z) .
\end{aligned}
$$

Setting $\left.F_{k}=\left[\int_{2^{-k}}^{2^{-k+1}}\left(t^{-\alpha} \mid \varphi(t \sqrt{L})\right) f(z) \mid\right)^{q} \frac{d t}{t}\right]^{1 / q}$ and using Lemma 2.2, we deduce that

$$
\begin{aligned}
{\left[\int_{2^{-j}}^{2^{-j+1}}\left(t^{-\alpha}\left|\varphi_{\lambda}^{*}(t \sqrt{L}) f(x)\right|\right)^{q} \frac{d t}{t}\right]^{r / q} } & \lesssim \sum_{k \geqslant j} 2^{(j-k)(A-\lambda+\alpha) r} \mathcal{M}_{r}\left(F_{k}\right)(x)^{r} \\
& \lesssim\left[\sum_{k \geqslant j} 2^{(j-k)(A-\lambda+\alpha) r} \mathcal{M}_{r}\left(F_{k}\right)(x)^{q}\right]^{r / q}
\end{aligned}
$$

where, in the last inequality, we use Hölder's inequality and the fact that $A-\lambda+$ $\alpha>0$. Hence, by applying (10), we obtain the desired estimate

$$
\left\|\left(\int_{0}^{\infty}\left[t^{-\alpha}\left|\varphi_{\lambda}^{*}(t \sqrt{L}) f\right|\right]^{q} \frac{d t}{t}\right)\right\|_{p, w} \lesssim\left\|\left(\int_{0}^{\infty}\left[t^{-\alpha}|\varphi(t \sqrt{L}) f|\right]^{q} \frac{d t}{t}\right)\right\|_{p, w} .
$$

Combining the results in Step 1, Step 2 and Step 3, we complete the proof of the theorem.

REMARK 3.7. (a) It is interesting to note that when $L=-\Delta$, the Laplacian on $\mathbb{R}^{n}, \mathscr{P}$ is the set of all polynomials. In this situation, Theorem 3.6 are in line with findings in [11].

(b) The presence of the polynomial $\rho$ in (51) and (52) can be omitted if $f \in L^{2}$. Indeed, in this case, instead of (54), we have, by the spectral theory,

$$
\left.\varphi(t \sqrt{L}) f=c_{\psi} \int_{0}^{\infty} \varphi(t \sqrt{L}) \psi(s \sqrt{L}) f \frac{d s}{s} \quad \text { (in } L^{2} \text { and hence in } \mathcal{S}^{\prime}\right) .
$$

Arguing similarly to the proof of the first inequalities in (51) and (52), we get the desired estimates. 
We denote $\Psi_{m, t}(L)=\left(t^{2} L\right)^{m} e^{-t^{2} L}$ for $t>0$ and $m \in \mathbb{N}$. For $\lambda>0$, we define

$$
\Psi_{m, t, \lambda}^{*}(L) f(x)=\sup _{y \in X} \frac{\left|\Psi_{m, t}(L) f(y)\right|}{(1+d(x, y) / t)^{\lambda}}
$$

for $f \in \mathcal{S}^{\prime}$.

Applying Theorem 3.6 and Remark 3.7 for $\varphi(\xi)=\xi^{2 m} e^{-\xi^{2}}$, we have the following heat kernel characterizations for the new Besov and Triebel-Lizorkin spaces.

Corollary 3.8. Let $w \in A_{\infty}, \alpha \in \mathbb{R}$ and $m>\alpha / 2$. Then the following norm equivalences hold:

(a) For $0<p, q \leqslant \infty, \lambda>n q_{w} / p$ and $f \in \mathcal{S}^{\prime}$, there exists $\rho \in \mathscr{P}$ so that

$$
\begin{aligned}
& \left(\int_{0}^{\infty}\left[t^{-\alpha}\left\|\Psi_{m, t, \lambda}^{*}(L)(f-\rho)\right\|_{p, w}\right]^{q} \frac{d t}{t}\right)^{1 / q} \lesssim\|f\|_{\dot{B}_{p, q, w}^{\alpha, L}} \\
& \quad \lesssim\left(\int_{0}^{\infty}\left[t^{-\alpha}\left\|\Psi_{m, t}(L) f\right\|_{p, w}\right]^{q} \frac{d t}{t}\right)^{1 / q}
\end{aligned}
$$

(b) For $0<p<0,0<q \leqslant \infty, \lambda>\max \left\{n / q, n q_{w} / p\right\}$ and $f \in \mathcal{S}^{\prime}$, there exists $\rho \in \mathscr{P}$ so that

$$
\begin{aligned}
& \left\|\left(\int_{0}^{\infty}\left[t^{-\alpha} \Psi_{m, t, \lambda}^{*}(L)(f-\rho)\right]^{q} \frac{d t}{t}\right)^{1 / q}\right\|_{p, w} \lesssim\|f\|_{\dot{F}_{p, q, w}^{\alpha, L}} \\
& \quad \lesssim\left\|\left(\int_{0}^{\infty}\left[t^{-\alpha} \Psi_{m, t}(L) f\right]^{q} \frac{d t}{t}\right)^{1 / q}\right\|_{p, w} .
\end{aligned}
$$

Moreover, if $f \in L^{2}, \rho$ can be removed in (62) and (63).

REMARK 3.9. Note that in [36, 50], the authors proved (62) for $s \in \mathbb{R}, 1 \leqslant p$ $\leqslant \infty, 0<q \leqslant \infty$ and (63) for $s \in \mathbb{R}, 1<p<\infty, 1<q \leqslant \infty$ for inhomogeneous and homogeneous Besov and Triebel-Lizorkin spaces under additional conditions of Hölder continuity and Markov property of the heat kernel $p_{t}(x, y)$. Moreover, their results are formulated for distributions in $\mathcal{S}_{\infty}^{\prime}$. Hence, Corollary 3.8 can be viewed as a significant extension of those results in $[36,50]$.

We end this subsection by a remark on an interesting extension of Theorem 3.6.

REMARK 3.10. The nondegeneracy condition of the function $\varphi$ in the definition of the class $\mathscr{S}_{m}(\mathbb{R}), \varphi(\xi) \neq 0$ on $(-2,-1 / 2) \cup(1 / 2,2)$, can be weakened 
to $\varphi(\lambda) \neq 0$ for some $\lambda>0$ (as $\varphi$ is even, this implies also $\varphi(-\lambda) \neq 0$ ). Then Theorem 3.6 holds under this weaker condition on $\varphi$. The proof of this stronger result can be done by modifying the proof for Theorem 3.6 and using the following two observations:

(a) There exist $a>0, c>b>0$ and even functions $\phi, \eta \in \mathscr{S}(\mathbb{R})$, such that $\operatorname{supp} \phi \subset[-\mathrm{a}, \mathrm{a}]$, supp $\eta \subset[-\mathrm{c},-\mathrm{b}] \cup[\mathrm{b}, \mathrm{c}]$ and

$$
\phi(\lambda)+\int_{1}^{\infty} \varphi(s \lambda) \eta(s \lambda) \frac{d s}{s}=1 \quad \forall \lambda \in \mathbb{R} .
$$

See, for example, $[43,58]$.

(b) Part (a) and an argument similar to the proof of Proposition 2.10 imply a Calderón reproducing formula

$$
f=\phi(t \sqrt{L} f)+\int_{1}^{\infty} \varphi(t s \sqrt{L} f) \eta(t s \sqrt{L}) \frac{d s}{s} \text { in } \mathcal{S}^{\prime},
$$

for all $f \in \mathcal{S}^{\prime}$ and all $t>0$.

We leave the details to the interested reader and also refer to $[11,12]$ for the proof in the classical case.

Theorem 3.6 and this remark essentially solve in the current setting a natural open problem posed in [9, Remark, pages 2480-2481], for an important class of 'kernel functions' and for the full range of indices.

\subsection{Characterizations for Triebel-Lizorkin spaces via Lusin functions and} the Littlewood-Paley functions. For $\alpha \in \mathbb{R}, \lambda, a>0$ and $0<q<\infty$, we define the Lusin function and the Littlewood-Paley function by setting

$$
\mathcal{G}_{\lambda, q}^{\alpha} F(x)=\left[\int_{0}^{\infty} \int_{X}\left(t^{-\alpha}|F(y, t)|\right)^{q}\left(1+\frac{d(x, y)}{t}\right)^{-\lambda q} \frac{d \mu(y) d t}{t V(x, t)}\right]^{1 / q}
$$

and

$$
\mathcal{S}_{a, q}^{\alpha} F(x)=\left[\int_{0}^{\infty} \int_{d(x, y)<a t}\left(t^{-\alpha}|F(y, t)|\right)^{q} \frac{d \mu(y) d t}{t V(x, t)}\right]^{1 / q}
$$

for every measurable function $F$, respectively.

When either $\alpha=0$ or $a=1$, we will drop them in the notation of $\mathcal{S}_{a, q}^{\alpha}$ and $\mathcal{G}_{\lambda, q}^{\alpha}$. We now have the following result regarding the estimates on the change of the angles for the function $\mathcal{S}_{a, q}^{\alpha}$. 
Proposition 3.11. Let $a>1, w \in A_{r}, 1 \leqslant r<\infty, 0<q<\infty, \alpha \in \mathbb{R}$ and $0<p<\infty$. Then there exists a constant $C$ so that

$$
\left\|\mathcal{S}_{a, q}^{\alpha} F\right\|_{p, w} \leqslant C a^{\gamma(r, p, q)}\left\|\mathcal{S}_{q}^{\alpha} F\right\|_{p, w}
$$

for all $F$, where

$$
\gamma(r, p, q)= \begin{cases}\frac{\tilde{n}+r n}{p \wedge q}, & p \geqslant q \\ \frac{2 n}{q}\left(1-\frac{p}{q}\right), & p<q .\end{cases}
$$

Proof. It suffices to prove the proposition for $\alpha=0$ and $q=2$; since in the general case of $\alpha$ and $q$, we set $\tilde{F}(y, t)=\left(t^{-\alpha}|F(y, t)|\right)^{q / 2}$ and then apply the result for the case $\alpha=0$ and $q=2$, we will get the desired estimate.

For the first case when $\alpha=0, q=2$ and $0<p<2$, we can adapt the proof of [1, Theorem 1] to our setting easily. Hence, we omit the details.

It remains to give the proof for the case $\alpha=0, q=2$ and $p \geqslant 2$. Indeed, for a positive function $g \in L_{w}^{(p / 2)^{\prime}}$, we have, by using (3),

$$
\begin{aligned}
\left\langle\left[\mathcal{S}_{a, 2} F\right]^{2}, g\right\rangle_{w}: & =\int_{X} \int_{0}^{\infty} \int_{d(x, y)<a t}|F(y, t)|^{2} \frac{d \mu(y) d t}{t V(x, t)} g(x) w(x) d \mu(x) \\
& \lesssim a^{\tilde{n}} \int_{X} \int_{0}^{\infty} \int_{d(x, y)<a t}|F(y, t)|^{2} \frac{d \mu(y) d t}{t V(y, t)} g(x) w(x) d \mu(x) \\
& =a^{\tilde{n}} \int_{X} \int_{0}^{\infty}|F(y, t)|^{2} M_{a t, w} g(y) w(B(y, a t)) \frac{d \mu(y) d t}{t V(y, t)}
\end{aligned}
$$

where

$$
M_{a t, w} g(y)=\frac{1}{w(B(y, a t))} \int_{B(y, a t)} g(x) w(x) d \mu(x) .
$$

Assume, for simplicity of writing (involving some constants), that $d$ is a metric. We then observe that

$$
\chi_{B(y, a t)}(x) \leqslant \frac{1}{w(B(y, t))} \int_{B(y, t)} \chi_{B(x, 2 a t)}(z) w(z) d \mu(z) .
$$

It follows

$$
\begin{aligned}
& \frac{1}{w(B(y, a t))} \int_{B(y, a t)} \chi_{B(y, a t)}(x) g(x) w(x) d \mu(x) \\
& \leqslant \frac{1}{w(B(y, t))} \int_{B(y, t)} \frac{1}{w(B(y, a t))}
\end{aligned}
$$




$$
\times \int_{B(y, a t)} \chi_{B(x, 2 a t)}(z) g(x) w(x) d \mu(x) w(z) d \mu(z) .
$$

Note that in this situation, we have $d(y, z) \leqslant t$ and $d(x, y) \leqslant a t$, and, hence, $B(y, a t) \subset B(z, 4 a t), B(x, 2 a t) \subset B(z, 4 a t)$ and $w(B(y, a t)) \sim w(B(z, 4 a t))$. As a consequence, we have

$$
\begin{aligned}
& \frac{1}{w(B(y, a t))} \int_{B(y, a t)} \chi_{B(x, 2 a t)}(z) g(x) w(x) d \mu(x) \\
& \quad \lesssim \frac{1}{w(B(z, 4 a t))} \int_{B(z, 4 a t)} g(x) w(x) d \mu(x) \\
& \quad \lesssim \mathcal{M}_{w} g(z) .
\end{aligned}
$$

This implies that

$$
M_{a t, w} g(y) \lesssim M_{t, w}\left[\mathcal{M}_{w} g\right](y)
$$

Hence,

$$
\begin{aligned}
& \left\langle\left[\mathcal{S}_{a, 2} F\right]^{2}, g\right\rangle_{w} \lesssim a^{\tilde{n}} \int_{X} \int_{0}^{\infty}|F(y, t)|^{2} M_{t, w}\left[\mathcal{M}_{w} g\right](y) w(B(y, a t)) \frac{d \mu(y) d t}{t V(y, t)} \\
& \quad=a^{\tilde{n}} \int_{X} \int_{0}^{\infty} \int_{d(x, y)<t}|F(y, t)|^{2} \frac{w(B(y, a t))}{w(B(y, t))} \frac{d \mu(y) d t}{t V(y, t)} \mathcal{M}_{w} g(x) w(x) d \mu(x) \\
& \quad \lesssim a^{\tilde{n}+n r} \int_{X} \int_{0}^{\infty} \int_{d(x, y)<t}|F(y, t)|^{2} \frac{d \mu(y) d t}{t V(y, t)} \mathcal{M}_{w} g(x) w(x) d \mu(x) \\
& \quad=a^{\tilde{n}+n r}\left\langle\left[\mathcal{S}_{2} F\right]^{2}, \mathcal{M}_{w} g\right\rangle_{w} \\
& \quad \leqslant a^{\tilde{n}+n r}\left\|\left[\mathcal{S}_{2} F\right]^{2}\right\|_{\frac{p}{2}, w}\left\|\mathcal{M}_{w} g\right\|_{\left(\frac{p}{2}\right)^{\prime}, w} \lesssim a^{\tilde{n}+n r}\left\|\mathcal{S}_{2} F\right\|_{p, w}^{2}\|g\|_{\left(\frac{p}{2}\right)^{\prime}, w} .
\end{aligned}
$$

Taking the supremum over all $g \in L_{w}^{(p / 2)^{\prime}}$ with $\|g\|_{(p / 2)^{\prime}, w} \leqslant 1$, we obtain

$$
\left\|\mathcal{S}_{a, 2} F\right\|_{p, w} \lesssim a^{(\tilde{n}+n r) / 2}\left\|\mathcal{S}_{2} F\right\|_{p, w} .
$$

This completes our proof.

We have the following corollary.

Corollary 3.12. Let $a \geqslant 1, w \in A_{r}, 1 \leqslant r<\infty, 0<q<\infty, \alpha \in \mathbb{R}$ and $0<p<\infty$. Then there exists a constant $C$ so that

$$
C^{-1}\left\|\mathcal{G}_{\lambda, q}^{\alpha} F\right\|_{p, w} \leqslant\left\|\mathcal{S}_{a, q}^{\alpha} F\right\|_{p, w} \leqslant C\left\|\mathcal{G}_{\lambda, q}^{\alpha} F\right\|_{p, w}
$$

for all $F$ provided that $\lambda>\gamma(r, p, q)$, where $\gamma(r, p, q)$ is defined by (66). 
Proof. Due to Proposition 3.11, we need only to prove the corollary for $a=1$. Since $\mathcal{S}_{a, q}^{\alpha} F \leqslant \mathcal{G}_{\lambda, q}^{\alpha} F$ for any $\lambda>0$, it suffices to prove that

$$
\left\|\mathcal{G}_{\lambda, q}^{\alpha} F\right\|_{p, w} \lesssim\left\|\mathcal{S}_{q}^{\alpha} F\right\|_{p, w} .
$$

Indeed, it is easy to see that

$$
\left[\mathcal{G}_{\lambda, q}^{\alpha} F\right]^{q} \leqslant \sum_{k \geqslant 0} 2^{-k q \lambda}\left[\mathcal{S}_{2^{k}, q}^{\alpha} F\right]^{q}
$$

Hence,

$$
\left[\mathcal{G}_{\lambda, q}^{\alpha} F\right]^{p} \leqslant\left[\sum_{k \geqslant 0} 2^{-k q \lambda}\left[\mathcal{S}_{2^{k}, q}^{\alpha} F\right]^{q}\right]^{p / q} .
$$

If $p / q<1$, we then have

$$
\left[\mathcal{G}_{\lambda, q}^{\alpha} F\right]^{p} \leqslant \sum_{k \geqslant 0} 2^{-k p \lambda}\left[\mathcal{S}_{2^{k}, q}^{\alpha} F\right]^{p} .
$$

This, along with Proposition 3.11, implies that

$$
\begin{aligned}
\left\|\mathcal{G}_{\lambda, q}^{\alpha} F\right\|_{p, w}^{p} & \leqslant \sum_{k \geqslant 0} 2^{-k p \lambda}\left\|\mathcal{S}_{2^{k}, q}^{\alpha} F\right\|_{p, w}^{p} \leqslant c \sum_{k \geqslant 0} 2^{-k p \lambda} 2^{k p \gamma(r, p, q)}\left\|\mathcal{S}_{q}^{\alpha} F\right\|_{p, w}^{p} \\
& \lesssim\left\|\mathcal{S}_{q}^{\alpha} F\right\|_{p, w}^{p}
\end{aligned}
$$

as long as $\lambda>\gamma(r, p, q)$.

If $p / q \geqslant 1$, then from (67), we have

$$
\left\|\left[\mathcal{G}_{\lambda, q}^{\alpha} F\right]^{q}\right\|_{p / q, w} \leqslant \sum_{k \geqslant 0} 2^{-k q \lambda}\left\|\left[\mathcal{S}_{2^{k}, q}^{\alpha} F\right]^{q}\right\|_{p / q, w} .
$$

Applying Proposition 3.11, we get

$$
\begin{aligned}
\left\|\mathcal{G}_{\lambda, q}^{\alpha} F\right\|_{p, w}^{q} & \leqslant \sum_{k \geqslant 0} 2^{-k q \lambda}\left\|\mathcal{S}_{2^{k}, q}^{\alpha} F\right\|_{p, w}^{q} \lesssim \sum_{k \geqslant 0} 2^{-k q \lambda} 2^{k p \gamma(r, p, q)}\left\|\mathcal{S}_{q}^{\alpha} F\right\|_{p, w}^{q} \\
& \lesssim\left\|\mathcal{S}_{q}^{\alpha} F\right\|_{p, w}^{q}
\end{aligned}
$$

provided $\lambda>\gamma(r, p, q)$.

This completes our proof of the corollary.

We have the following characterization. 
Proposition 3.13. Let $\psi$ be a partition of unity. Then for $w \in A_{\infty}, 0<p$, $q<\infty$ and $\alpha \in \mathbb{R}$, we have

$$
\|f\|_{\dot{F}_{p, q, w}^{\alpha, L}} \sim\left\|\mathcal{G}_{\lambda, q}^{\alpha}(\psi(t \sqrt{L}) f)\right\|_{p, w} \sim\left\|\mathcal{S}_{q}^{\alpha}(\psi(t \sqrt{L}) f)\right\|_{p, w}
$$

for all $f \in \mathcal{S}_{\infty}^{\prime}$, provided that $\lambda>\gamma\left(q_{w}, p, q\right)$, where $\gamma\left(q_{w}, p, q\right)$ is defined by (66).

Proof. We first prove that $\left\|\mathcal{S}_{q}^{\alpha}(\psi(t \sqrt{L}) f)\right\|_{p, w} \lesssim\|f\|_{\dot{F}_{p, q, w}^{\alpha, L}}$. First observe that

$$
|\psi(t \sqrt{L}) f(y)| \leqslant \psi_{\lambda}^{*}(t \sqrt{L}) f(x)
$$

for all $\lambda>0$ and $d(x, y)<t$. Therefore,

$$
\begin{aligned}
\mathcal{S}_{q}^{\alpha}(\psi(t \sqrt{L}) f)(x) & \leqslant\left[\int_{0}^{\infty} \int_{d(x, y)<t}\left(t^{-\alpha}\left|\psi_{\lambda}^{*}(t \sqrt{L}) f(x)\right|\right)^{q} \frac{d \mu(y) d t}{t V(x, t)}\right]^{1 / q} \\
& \lesssim\left[\int_{0}^{\infty}\left(t^{-\alpha}\left|\psi_{\lambda}^{*}(t \sqrt{L}) f(x)\right|\right)^{q} \frac{d t}{t}\right]^{1 / q} .
\end{aligned}
$$

This, along with Theorem 3.5, implies that

$$
\left\|\mathcal{S}_{q}^{\alpha}(\psi(t \sqrt{L}) f)\right\|_{p, w} \lesssim\|f\|_{\dot{F}_{p, q, w}^{\alpha, L}} .
$$

Due to the above estimate and Corollary 3.12, it remains to show that

$$
\|f\|_{\dot{F}_{p, q, w}^{\alpha, L}} \lesssim\left\|\mathcal{G}_{\lambda, q}^{\alpha}(\psi(t \sqrt{L}) f)\right\|_{p, w} .
$$

By Proposition 2.17, we have

$$
|\psi(t \sqrt{L}) f(x)| \lesssim\left[\int_{X} \frac{1}{V(x, t)} \frac{|\psi(t \sqrt{L}) f(z)|^{q}}{(1+d(x, z) / t)^{\lambda q}} d \mu(z)\right]^{1 / q}
$$

for all $x \in X, \lambda>0$ and $t>0$.

This implies that

$$
\left\|\left(\int_{0}^{\infty}\left(t^{-\alpha}|\psi(t \sqrt{L}) f|\right)^{q} \frac{d t}{t}\right)^{1 / q}\right\|_{p, w} \lesssim\left\|\mathcal{G}_{\lambda, q}^{\alpha}(\psi(t \sqrt{L}) f)\right\|_{p, w} .
$$

Using Theorem 3.5, we obtain the bound

$$
\|f\|_{\dot{F}_{p, q, w}^{\alpha, L}} \lesssim\left\|\mathcal{G}_{\lambda, q}^{\alpha}(\psi(t \sqrt{L}) f)\right\|_{p, w}
$$

as desired. 
We also have a similar square function characterization for the new TriebelLizorkin spaces via functions in $\mathscr{S}_{m}(\mathbb{R})$.

Proposition 3.14. Let $w \in A_{\infty}, 0<p<\infty, 0<q<\infty, \alpha \in \mathbb{R}, \lambda>\gamma\left(q_{w}\right.$, $p, q)$ and $\varphi \in \mathscr{S}_{m}(\mathbb{R})$ with $m>\alpha / 2$. Then for each $f \in \mathcal{S}^{\prime}$, there exists $\rho \in \mathscr{P}$ such that

$$
\begin{aligned}
& \left\|\mathcal{G}_{\lambda, q}^{\alpha}(\varphi(t \sqrt{L})(f-\rho))\right\|_{p, w} \sim\left\|\mathcal{S}_{q}^{\alpha}(\varphi(t \sqrt{L})(f-\rho))\right\|_{p, w} \\
& \quad \lesssim\|f\|_{\dot{F}_{p, q, w}^{\alpha, L}} \lesssim\left\|\mathcal{G}_{\lambda, q}^{\alpha}(\varphi(t \sqrt{L}) f)\right\|_{p, w} \sim\left\|\mathcal{S}_{q}^{\alpha}(\varphi(t \sqrt{L}) f)\right\|_{p, w} .
\end{aligned}
$$

Moreover, the distribution $\rho$ in the inequalities above can be removed if $f \in L^{2}$.

Proof. Arguing similarly to the proof of Proposition 3.13 and using Theorem 3.6, we show that

$$
\left\|\mathcal{S}_{q}^{\alpha}(\varphi(t \sqrt{L})(f-\rho))\right\|_{p, w} \lesssim\|f\|_{\dot{F}_{p, q, w}^{\alpha, L}} .
$$

It remains to prove that

$$
\|f\|_{\dot{F}_{p, q, w}^{\alpha, L}} \lesssim\left\|\mathcal{G}_{\lambda, q}^{\alpha}(\varphi(t \sqrt{L}) f)\right\|_{p, w} .
$$

To do this, we divide into two cases.

If $q \in(0,1]$, then applying (60) with $\lambda$ replaced by $\lambda+\tilde{n} / q, r=q$ and $A-$ $\lambda-\tilde{n} / q+\alpha>0$ and using (3), we deduce that

$$
\begin{aligned}
& \sum_{j \in \mathbb{Z}} \int_{2^{-j}}^{2^{-j+1}}\left(t^{-\alpha}|\varphi(t \sqrt{L}) f(x)|\right)^{q} \frac{d t}{t} \\
& \quad \lesssim \sum_{j \in \mathbb{Z}} \sum_{k \in \mathbb{Z}} \int_{2^{-k}}^{2^{-k+1}} \int_{X} \frac{2^{-|k-j|(A-\lambda-\tilde{n} / q+\alpha) q}}{V(x, t)} \frac{\left(t^{-\alpha}|\varphi(t \sqrt{L}) f(z)|\right)^{q}}{(1+d(x, z) / t)^{\lambda q}} d \mu(z) \frac{d t}{t} .
\end{aligned}
$$

Hence,

$$
\int_{0}^{\infty}\left(t^{-\alpha}|\varphi(t \sqrt{L}) f(x)|\right)^{q} \frac{d t}{t} \lesssim \int_{0}^{\infty} \int_{X} \frac{1}{V(x, t)} \frac{\left(t^{-\alpha}|\varphi(t \sqrt{L}) f(z)|\right)^{q}}{(1+d(x, z) / t)^{\lambda q}} d \mu(z) \frac{d t}{t}
$$

As a consequence,

$$
\left[\int_{0}^{\infty}\left(t^{-\alpha}|\varphi(t \sqrt{L}) f(x)|\right)^{q} \frac{d t}{t}\right]^{1 / q} \lesssim \mathcal{G}_{\lambda, q}^{\alpha}(\varphi(t \sqrt{L}) f)(x) .
$$

This, along with Theorem 3.6, yields (68). 
If $q>1$, then for a partition of unity function $\psi$, we have

$$
f=c \int_{0}^{\infty} \psi(s \sqrt{L}) \varphi(s \sqrt{L}) f \frac{d s}{s} \text { in } \mathcal{S}_{\infty}^{\prime} .
$$

It follows that, for each $t>0$ and $x \in X$,

$$
\psi(t \sqrt{L}) f(x)=c \int_{t / 4}^{4 t} \psi(t \sqrt{L}) \psi(s \sqrt{L}) \varphi(s \sqrt{L}) f(x) \frac{d s}{s} .
$$

Using Lemma 2.6, for $\lambda>\frac{n q_{w}}{p \wedge q}$, we see that

$$
\begin{aligned}
& |\psi(t \sqrt{L}) f(x)| \\
& \quad \lesssim \int_{t / 4}^{4 t} \int_{X} \frac{1}{V(x \vee y, s)}\left(\frac{s}{s+d(x, y)}\right)^{\lambda q}|\varphi(s \sqrt{L}) f(y)| d \mu(y) \frac{d s}{s} .
\end{aligned}
$$

Hölder's inequality and Lemma 2.2 then imply that

$$
\begin{aligned}
\mid \psi( & t \sqrt{L})\left.f(x)\right|^{q} \\
\lesssim & \int_{t / 4}^{4 t} \int_{X} \frac{1}{V(x \vee y, s)}\left(\frac{s}{s+d(x, y)}\right)^{\lambda q}|\varphi(s \sqrt{L}) f(y)|^{q} d \mu(y) \frac{d s}{s} \\
& \times\left[\int_{t / 4}^{4 t} \int_{X} \frac{1}{V(x \vee y, s)}\left(\frac{s}{s+d(x, y)}\right)^{\lambda q} d \mu(y) \frac{d s}{s}\right]^{q / q^{\prime}} \\
& \lesssim \int_{t / 4}^{4 t} \int_{X} \frac{1}{V(x \vee y, s)}\left(\frac{s}{s+d(x, y)}\right)^{\lambda q}|\varphi(s \sqrt{L}) f(y)|^{q} d \mu(y) \frac{d s}{s} .
\end{aligned}
$$

Consequently,

$$
\begin{aligned}
\int_{0}^{\infty} & \left(t^{-\alpha}|\psi(t \sqrt{L}) f(x)|\right)^{q} \frac{d t}{t} \\
\lesssim & \int_{0}^{\infty} \int_{t / 4}^{4 t} \int_{X} \frac{1}{V(x \vee y, s)}\left(\frac{s}{s+d(x, y)}\right)^{\lambda q} \\
& \times\left(s^{-\alpha}|\varphi(s \sqrt{L}) f(y)|\right)^{q} d \mu(y) \frac{d s}{s} \frac{d t}{t} \\
\lesssim & \int_{0}^{\infty} \int_{s / 4}^{4 s} \int_{X} \frac{1}{V(x \vee y, s)}\left(\frac{s}{s+d(x, y)}\right)^{\lambda q} \\
& \times\left(s^{-\alpha}|\varphi(s \sqrt{L}) f(y)|\right)^{q} d \mu(y) \frac{d t}{t} \frac{d s}{s} \\
\lesssim & \int_{0}^{\infty} \int_{X} \frac{1}{V(x \vee y, s)}\left(\frac{s}{s+d(x, y)}\right)^{\lambda q}\left(s^{-\alpha}|\varphi(s \sqrt{L}) f(y)|\right)^{q} d \mu(y) \frac{d s}{s} \\
\lesssim & \mathcal{G}_{\lambda, q}^{\alpha}(\varphi(t \sqrt{L}) f)(x)^{q} .
\end{aligned}
$$


This, along with Theorem 3.5, yields (68).

This completes our proof.

Corollary 3.15. Let $w \in A_{\infty}, 0<p<\infty, 0<q<\infty, \alpha \in \mathbb{R}, \lambda>$ $\gamma\left(q_{w}, p, q\right)$ and $m>\alpha / 2$. Then for each $f \in \mathcal{S}^{\prime}$, there exists $\rho \in \mathscr{P}$ such that

$$
\begin{aligned}
& \left\|\mathcal{G}_{\lambda, q}^{\alpha}\left(\Psi_{m, t}(L)(f-\rho)\right)\right\|_{p, w} \sim\left\|\mathcal{S}_{q}^{\alpha}\left(\Psi_{m, t}(L)(f-\rho)\right)\right\|_{p, w} \\
& \quad \lesssim\|f\|_{\dot{F}_{p, q, w}^{\alpha, L}} \lesssim\left\|\mathcal{G}_{\lambda, q}^{\alpha}\left(\Psi_{m, t}(L) f\right)\right\|_{p, w} \sim\left\|\mathcal{S}_{q}^{\alpha}\left(\Psi_{m, t}(L) f\right)\right\|_{p, w},
\end{aligned}
$$

where $\Psi_{m, t}(L)=\left(t^{2} L\right)^{m} e^{-t^{2} L}$.

Moreover, the distribution $\rho$ can be removed if $f \in L^{2}$.

3.5. Weighted Triebel-Lizorkin space $\dot{\boldsymbol{F}}_{\infty, q, w}^{\alpha, L}$ and its characterizations. In this section, we will give the definition of the weighted Triebel-Lizorkin space $\dot{F}_{\infty, q, w}^{\alpha, L}$ and prove some characterizations for this space.

Definition 3.16. Let $0<q \leqslant \infty, \alpha \in \mathbb{R}$ and $w \in A_{\infty}$. Let $\psi$ be a partition of unity. The space $\dot{F}_{\infty, q, w}^{\alpha, L}$ is defined as the set of all $f \in \mathcal{S}_{\infty}^{\prime}$ so that

$$
\|f\|_{F_{\infty, q, w}^{\alpha, \psi, L}}=\sup _{Q: \text { balls }}\left(\frac{V(Q)}{w(Q)^{2}} \int_{Q} \sum_{j \geqslant-\log _{2} r_{Q}}^{\infty}\left(2^{j \alpha}\left|\psi_{j}(\sqrt{L}) f(x)\right|\right)^{q} d \mu(x)\right)^{1 / q},
$$

where the supremum is taken over all balls $Q$ in $X$ with radius $r_{Q}$, with the interpretation that when $q=\infty$, one has

$$
\|f\|_{\dot{F}_{\infty, \infty, w}^{\alpha, \psi, L}}=\sup _{Q: \text { balls } j \geqslant-\log _{2} r_{Q}}\left(\frac{V(Q)}{w(Q)^{2}} \int_{Q} 2^{j \alpha}\left|\psi_{j}(\sqrt{L}) f(x)\right| d \mu(x)\right) .
$$

Proposition 3.17. Let $0<q \leqslant \infty, \alpha \in \mathbb{R}$ and $w \in A_{\infty}$. Let $\psi$ and $\varphi$ be partitions of unity. Then the spaces $\dot{F}_{\infty, q, w}^{\alpha, \psi, L}$ and $\dot{F}_{\infty, q, w}^{\alpha, \varphi}$ are equivalent. Hence, we define the space $\dot{F}_{\infty, q, w}^{\alpha, L}$ as any space $\dot{F}_{\infty, q, w}^{\alpha, \varphi, L}$ with $\varphi$ being a partition of unity.

The proposition is a direct consequence of Lemmas 3.18 and 3.19.

Lemma 3.18. Let $0<q \leqslant \infty, \alpha \in \mathbb{R}$ and $w \in A_{p}$ with $1<p<\infty$. Let $\psi$ and $\varphi$ be partitions of unity. Then for each $\lambda>n p / q+2 n p^{2} / q$, we have

$$
\sup _{Q: \text { balls }}\left(\frac{V(Q)}{w(Q)^{2}} \int_{Q} \sum_{j \geqslant-\log _{2} r_{Q}}^{\infty}\left(2^{j \alpha}\left|\psi_{j, \lambda}^{*}(\sqrt{L}) f(x)\right|\right)^{q} d \mu(x)\right)^{1 / q}
$$




$$
\sim \sup _{Q: \text { balls }}\left(\frac{V(Q)}{w(Q)^{2}} \int_{Q} \sum_{j \geqslant-\log _{2} r_{Q}}^{\infty}\left(2^{j \alpha}\left|\varphi_{j, \lambda}^{*}(\sqrt{L}) f(x)\right|\right)^{q} d \mu(x)\right)^{1 / q} .
$$

Proof. The proposition follows easily from (36).

Lemma 3.19. Let $0<q \leqslant \infty, \alpha \in \mathbb{R}$ and $w \in A_{p}, 1<p<\infty$. Let $\psi$ be a partition of unity. Then for each $\lambda>n p / q+2 n p^{2} / q$, we have the norm equivalence:

$$
\sup _{Q: \text { balls }}\left(\frac{V(Q)}{w(Q)^{2}} \int_{Q} \sum_{j \geqslant-\log _{2} r_{Q}}^{\infty}\left(2^{j \alpha}\left|\psi_{j, \lambda}^{*}(\sqrt{L}) f(x)\right|\right)^{q} d \mu(x)\right)^{1 / q} \sim\|f\|_{\dot{F}_{\infty, q, w}^{\alpha, \psi, L}} .
$$

Proof. We consider the case $0<q<\infty$. It suffices to prove

$$
\sup _{Q: \text { balls }}\left(\frac{V(Q)}{w(Q)^{2}} \int_{Q} \sum_{j \geqslant-\log _{2} r_{Q}}^{\infty}\left(2^{j \alpha}\left|\psi_{j, \lambda}^{*}(\sqrt{L}) f(x)\right|\right)^{q} d \mu(x)\right)^{1 / q} \lesssim\|f\|_{\dot{F}_{\infty, q, w}^{\alpha, \psi, L}} .
$$

Indeed, fix a ball $Q$ and let $x \in Q$. For $j \geqslant v$, applying Proposition 2.17 with $r=q / p$, we obtain

$$
\begin{aligned}
\left|\psi_{j, \lambda}^{*}(\sqrt{L}) f(x)\right|^{q} \lesssim & {\left[\int_{X} \frac{1}{V\left(z, 2^{-j}\right)} \frac{\left|\psi_{j}(\sqrt{L}) f(z)\right|^{q / p}}{\left(1+2^{j} d(x, z)\right)^{\lambda q / p}} d \mu(z)\right]^{p} } \\
\lesssim & {\left[\int_{4 Q} \frac{1}{V\left(z, 2^{-j}\right)} \frac{\left|\psi_{j}(\sqrt{L}) f(z)\right|^{q / p}}{\left(1+2^{j} d(x, z)\right)^{\lambda q / p}} d \mu(z)\right]^{p} } \\
& +\left[\int_{X \backslash 4 Q} \frac{1}{V\left(z, 2^{-j}\right)} \frac{\left|\psi_{j}(\sqrt{L}) f(z)\right|^{q / p}}{\left(1+2^{j} d(x, z)\right)^{\lambda q / p}} d \mu(z)\right]^{p} \\
:= & E_{j, 1}(x)+E_{j, 2}(x) .
\end{aligned}
$$

Using Lemma 2.2, we can bound $E_{j, 1}$ as follows:

$$
E_{j, 1}(x) \lesssim\left[\mathcal{M}_{r}\left(\left|\psi_{j}(\sqrt{L}) f\right| \chi_{4 Q}\right)(x)\right]^{q}
$$

Therefore,

$$
\begin{aligned}
& \frac{V(Q)}{w(Q)^{2}} \int_{Q} \sum_{j \geqslant-\log _{2} r_{Q}}^{\infty} 2^{j \alpha q} E_{j, 1}(x) d \mu(x) \\
& \quad \lesssim \frac{V(Q)}{w(Q)^{2}} \int_{Q} \sum_{j \geqslant-\log _{2} r_{Q}}^{\infty} 2^{j \alpha q}\left[\mathcal{M}_{r}\left(\left|\psi_{j}(\sqrt{L}) f\right| \chi_{4 Q}\right)(x)\right]^{q} d \mu(x) .
\end{aligned}
$$


Since $\mathcal{M}_{r}$ is bounded on $L_{w}^{q}(X)$, the inequality above and Fefferman-Stein's inequality imply that

$$
\begin{aligned}
& \frac{V(Q)}{w(Q)^{2}} \int_{Q} \sum_{j \geqslant-\log _{2} r_{Q}}^{\infty} 2^{j \alpha q} E_{j, 1}(x) d x \\
& \quad \lesssim \frac{V(Q)}{w(Q)^{2}} \int_{4 Q} \sum_{j \geqslant-\log _{2} r_{Q}}^{\infty}\left(\left|2^{j \alpha}\right| \psi_{j}(\sqrt{L}) f(x) \mid\right)^{q} d \mu(x) \\
& \quad \lesssim\|f\|_{\dot{F}_{\infty, q, w}^{\alpha, \psi, L}}^{q}
\end{aligned}
$$

We now use Hölder's inequality to dominate $E_{j, 2}(x)$ by

$$
\begin{aligned}
& {\left[\int_{X \backslash 4 Q} \frac{1}{V\left(z, 2^{-j}\right)} \frac{\left|\psi_{j}(\sqrt{L}) f(z)\right|^{q}}{\left(1+2^{j} d(x, z)\right)^{\lambda q / p}} d \mu(z)\right]} \\
& \quad \times\left[\int_{X \backslash 4 Q} \frac{1}{V\left(z, 2^{-j}\right)}\left(1+2^{j} d(x, z)\right)^{-\lambda q / p} d \mu(z)\right]^{p / p^{\prime}} .
\end{aligned}
$$

Recalling Lemma 2.2,

$$
\left[\int_{X \backslash 4 Q} \frac{1}{V\left(z, 2^{-j}\right)}\left(1+2^{j} d(x, z)\right)^{-\lambda q / p} d \mu(z)\right]^{p / p^{\prime}} \lesssim 1
$$

provided $\lambda>n p / q$.

Plugging this into (69) yields

$$
\begin{aligned}
E_{j, 2}(x) & \lesssim \sum_{k=3}^{\infty} \int_{S_{k}(Q)} \frac{1}{V\left(z, 2^{-j}\right)} \frac{\left|\psi_{j}(\sqrt{L}) f(z)\right|^{q}}{\left(1+2^{j} d(x, z)\right)^{\lambda q / p}} d \mu(z) \\
& \lesssim \sum_{k=3}^{\infty} 2^{-(j+k-v) \lambda q / p} \int_{S_{k}(Q)} \frac{1}{V\left(z, 2^{-j}\right)}\left|\psi_{j}(\sqrt{L}) f(z)\right|^{q} d \mu(z) \\
& \lesssim \sum_{k=3}^{\infty} \frac{2^{-(j+k-v)(\lambda q / p-n)}}{V\left(2^{k} Q\right)} \int_{S_{k}(Q)}\left|\psi_{j}(\sqrt{L}) f(z)\right|^{q} d \mu(z) \\
& \lesssim \sum_{k=3}^{\infty} \frac{2^{-k(\lambda q / p-n)}}{V\left(2^{k} Q\right)} \int_{S_{k}(Q)}\left|\psi_{j}(\sqrt{L}) f(z)\right|^{q} d \mu(z),
\end{aligned}
$$

where $v$ is the integer part of $-\log _{2} r_{Q}$, and in the last inequality we use the fact that $j \geqslant v$. Hence, using (5), we deduce that

$$
\frac{V(Q)}{w(Q)^{2}} \int_{Q} \sum_{j \geqslant-\log _{2} r_{Q}}^{\infty} 2^{j \alpha q} E_{j, 2}(x) d \mu(x)
$$




$$
\begin{aligned}
& \lesssim \sum_{k=3}^{\infty} \frac{2^{-k(\lambda q / p-n)}}{V\left(2^{k} Q\right)} \frac{V(Q)^{2}}{w(Q)^{2}} \int_{2^{k} Q} \sum_{j \geqslant v}^{\infty}\left(2^{j \alpha}\left|\psi_{j}(\sqrt{L}) f(z)\right|\right)^{q} d \mu(z) \\
& \lesssim \sum_{k=3}^{\infty} 2^{-k(\lambda q / p-n-2 n p)} \frac{V\left(2^{k} Q\right)}{w\left(2^{k} Q\right)^{2}} \int_{2^{k} Q} \sum_{j \geqslant v-k}^{\infty}\left(2^{j \alpha}\left|\psi_{j}(\sqrt{L}) f(z)\right|\right)^{q} d \mu(z) \\
& \lesssim \sum_{k \geqslant 0} 2^{-k(\lambda q / p-n-2 n p)}\|f\|_{\dot{F}_{\infty, q, w}^{\alpha, \psi}}^{q, L} \\
& \lesssim\|f\|_{\dot{F}_{\infty, q, w}^{\alpha, \psi, L}}^{q}
\end{aligned}
$$

as long as $\lambda>n p / q+2 n p^{2} / q$. Combining the estimates for $E_{j, 1}$ and $E_{j, 2}$, we conclude that

$$
\left(\frac{V(Q)}{w(Q)^{2}} \int_{Q} \sum_{j \geqslant-\log _{2} r_{Q}}^{\infty}\left(2^{j \alpha}\left|\psi_{j, \lambda}^{*}(\sqrt{L}) f(x)\right|\right)^{q} d \mu(x)\right)^{1 / q} \lesssim\|f\|_{\dot{F}_{\infty, q, w}^{\alpha, \psi, L}},
$$

which implies the desired result when $q<\infty$.

The proof for the case $q=\infty$ is similar with minor modifications, and, hence, we leave it to the reader.

Proposition 3.20. Let $0<q \leqslant \infty, \alpha \in \mathbb{R}$ and $w \in A_{p}, 1<p<\infty$. Let $\psi$ be a partition of unity. Then for $\lambda>n p / q+2 n p^{2} / q$, we have

$$
\begin{aligned}
\|f\|_{\dot{F}_{\infty, q, w}^{\alpha, L}} & \sim \sup _{x \in X, t>0}\left(\frac{V(x, t)}{w(B(x, t))^{2}} \int_{B(x, t)} \int_{0}^{t}\left(s^{-\alpha}|\psi(s \sqrt{L}) f(y)|\right)^{q} \frac{d s}{s} d \mu(y)\right)^{1 / q} \\
= & : N_{\alpha, q, L}(f) \\
& \sim \sup _{x \in X, t>0}\left(\frac{V(x, t)}{w(B(x, t))^{2}} \int_{B(x, t)} \int_{0}^{t}\left(s^{-\alpha}\left|\psi_{\lambda}^{*}(s \sqrt{L}) f(y)\right|\right)^{q} \frac{d s}{s} d \mu(y)\right)^{1 / q} \\
& =: N_{\lambda, \alpha, q, L}^{*}(f)
\end{aligned}
$$

for all $f \in \mathcal{S}_{\infty}^{\prime}$.

Proof. As usual, we will only prove the proposition for $0<q<\infty$. We first claim that

$$
\|f\|_{\dot{F}_{\infty, q, w}^{\alpha, L}} \lesssim N_{\lambda, \alpha, q, L}^{*}(f) .
$$

Indeed, from Proposition 2.18, we have, for every $j \in \mathbb{Z}$ and $x \in X$,

$$
\left|\psi_{j}(\sqrt{L}) f(x)\right|^{q} \lesssim \int_{2^{-j-2}}^{2^{-j+2}}\left|\psi_{\lambda}^{*}(s \sqrt{L}) f(x)\right|^{q} \frac{d s}{s}
$$


Hence, for any $v \in \mathbb{Z}$,

$$
\begin{aligned}
\sum_{j \geqslant v}\left(2^{j \alpha}\left|\psi_{j}(\sqrt{L}) f(x)\right|\right)^{q} & \lesssim \sum_{j \geqslant v} \int_{2^{-j-2}}^{2^{-j+2}}\left(s^{-\alpha}\left|\psi_{\lambda}^{*}(s \sqrt{L}) f(x)\right|\right)^{q} \frac{d s}{s} \\
& =\int_{0}^{2^{-v+2}}\left(s^{-\alpha}\left|\psi_{\lambda}^{*}(s \sqrt{L}) f(x)\right|\right)^{q} \frac{d s}{s} .
\end{aligned}
$$

This implies that

$$
\begin{aligned}
& \frac{V(Q)}{w(Q)^{2}} \int_{Q} \sum_{j \geqslant v}\left(2^{j \alpha}\left|\psi_{j}(\sqrt{L}) f(x)\right|\right)^{q} d \mu(x) \\
& \quad \lesssim \frac{V(Q)}{w(Q)^{2}} \int_{0}^{2^{-v+2}} \int_{Q}\left(s^{-\alpha}\left|\psi_{\lambda}^{*}(s \sqrt{L}) f(x)\right|\right)^{q} d \mu(x) \frac{d s}{s}
\end{aligned}
$$

for every ball $Q$. Hence, (70) follows.

We next prove that

$$
N_{\lambda, \alpha, q, L}^{*}(f) \lesssim N_{\alpha, q, L}(f) .
$$

To do this, we employ Proposition 2.17 to find that

$$
\left|\psi_{\lambda}^{*}(s \sqrt{L}) f(x)\right| \lesssim\left[\int_{X} \frac{1}{V(z, s)}\left(1+\frac{d(x, z)}{s}\right)^{-\lambda r}|\psi(s \sqrt{L}) f(z)|^{r} d \mu(z)\right]^{1 / r}
$$

where $r=q / p$. Fix a ball $Q=B\left(x_{1}, t\right)$. Then the above implies that, for every $x \in Q$,

$$
\begin{aligned}
\int_{0}^{t}\left(s^{-\alpha}\left|\psi_{\lambda}^{*}(s \sqrt{L}) f(x)\right|\right)^{q} \frac{d s}{s} \lesssim & \int_{0}^{t}\left[\int_{4 Q} \frac{1}{V(z, s)}\left(1+\frac{d(x, z)}{s}\right)^{-\lambda r}\right. \\
& \left.\times\left|s^{-\alpha} \psi(s \sqrt{L}) f(z)\right|^{r} d \mu(z)\right]^{q / r} \frac{d s}{s} \\
& +\int_{0}^{t}\left[\int_{X \backslash 4 Q} \frac{1}{V(z, s)}\left(1+\frac{d(x, z)}{s}\right)^{-\lambda r}\right. \\
& \left.\times\left|s^{-\alpha} \psi(s \sqrt{L}) f(z)\right|^{r} d \mu(z)\right]^{q / r} \frac{d s}{s} .
\end{aligned}
$$

Using the above estimates and an argument similar to the proof of Lemma 3.19, we obtain (71).

It remains to show that

$$
N_{\alpha, q, L}(f) \lesssim\|f\|_{\dot{F}_{\alpha, q, w}^{\alpha, L}}
$$


Observe that for $t \in\left(2^{-v-1}, 2^{-v}\right]$ with $v \in \mathbb{Z}$,

$$
\begin{aligned}
& \frac{V(x, t)}{w(B(x, t))^{2}} \int_{B(x, t)} \int_{0}^{t}\left(s^{-\alpha}|\psi(s \sqrt{L}) f(y)|\right)^{q} \frac{d s}{s} d y \\
& \lesssim \frac{V\left(x, 2^{-v}\right)}{w\left(B\left(x, 2^{-v}\right)\right)^{2}} \int_{B\left(x, 2^{-v}\right)} \int_{0}^{2^{-v}}\left(s^{-\alpha}|\psi(s \sqrt{L}) f(y)|\right)^{q} \frac{d s}{s} d y \\
& \lesssim \frac{V\left(x, 2^{-v}\right)}{w\left(B\left(x, 2^{-v}\right)\right)^{2}} \int_{B\left(x, 2^{-v}\right)} \sum_{j \geqslant v} \int_{2^{-j-1}}^{2^{-j}}\left(s^{-\alpha}|\psi(s \sqrt{L}) f(y)|\right)^{q} \frac{d s}{s} d y .
\end{aligned}
$$

By Proposition 2.16, for $\lambda>0$ and $s \in\left[2^{-j-1}, 2^{-j}\right]$, we have

$$
|\psi(s \sqrt{L}) f(x)| \lesssim \sum_{k=j-2}^{j+3} \psi_{k, \lambda}^{*} f(x), \quad x \in X .
$$

Substituting this into (73) and using Lemma 3.19, we obtain (72).

The conclusion of the proposition follows immediately from (70), (71) and (72).

THEOREM 3.21. Let $0<q \leqslant \infty, \alpha \in \mathbb{R}, m>\alpha / 2$ and $w \in A_{p}, 1 \leqslant p<\infty$. Let $\varphi \in \mathscr{S}_{m}(\mathbb{R})$. Assume $\lambda>n p / q+2 n p^{2} / q$.

(i) If $p=1$, then for each $f \in \mathcal{S}^{\prime}$, there exists $\rho \in \mathscr{P}$ so that

$$
\begin{aligned}
& K_{\lambda, \alpha, q, L}^{*}(f-\rho) \\
& \quad:=\sup _{x \in X, t>0}\left(\frac{V(x, t)}{w(B(x, t))^{2}} \int_{B(x, t)} \int_{0}^{t}\right. \\
& \left.\quad \times\left(s^{-\alpha}\left|\varphi_{\lambda}^{*}(s \sqrt{L})(f-\rho)(y)\right|\right)^{q} \frac{d s}{s} d \mu(y)\right)^{1 / q} \lesssim\|f\|_{\dot{F}_{\infty, q, w}^{\alpha, L}} .
\end{aligned}
$$

(ii) If $p>1$, then

$$
\begin{aligned}
& \|f\|_{\dot{F}_{\infty, q, w}^{\alpha, L}} \\
& \quad \lesssim \sup _{x \in X, t>0}\left(\frac{V(x, t)}{w(B(x, t))^{2}} \int_{B(x, t)} \int_{0}^{t}\left(s^{-\alpha}|\varphi(s \sqrt{L}) f(y)|\right)^{q} \frac{d s}{s} d \mu(y)\right)^{1 / q} \\
& \quad=: K_{\alpha, q, L}(f)
\end{aligned}
$$

for every $f \in \mathcal{S}^{\prime}$.

Moreover, the distribution $\rho$ in (74) can be omitted if $f \in L^{2}(X)$. 
Proof. We will only prove the theorem for $0<q<\infty$, as the case $q=\infty$ is similar and so we omit the details.

We prove (74) first. Recall from (55) that we can find $\rho \in \mathscr{P}$ such that for each $u \in\left[2^{-v-1}, 2^{-v}\right)$ for some $v \in \mathbb{Z}$ and $\lambda>0$,

$$
\left|\varphi_{\lambda}^{*}(u \sqrt{L})(f-\rho)\right| \lesssim \sum_{j \in \mathbb{Z}} 2^{-2 m|v-j|}\left|\psi_{j, \lambda}^{*}(\sqrt{L}) f\right| .
$$

It follows that if $q \leqslant 1$, then we have

$$
\int_{2^{-\nu-1}}^{2^{-v}}\left(u^{-\alpha}\left|\varphi_{\lambda}^{*}(u \sqrt{L})(f-\rho)\right|\right)^{q} \frac{d u}{u} \lesssim \sum_{j \in \mathbb{Z}} 2^{-q(2 m-\alpha)|v-j|}\left(2^{j \alpha}\left|\psi_{j, \lambda}^{*}(\sqrt{L}) f\right|\right)^{q}
$$

for every $v \in \mathbb{Z}$. Therefore, for any $t>0$ so that $t \in\left[2^{-v_{0}-1}, 2^{-v_{0}}\right)$, and $x \in X$, we have

$$
\begin{aligned}
& \frac{V(x, t)}{w(B(x, t))^{2}} \int_{B(x, t)} \int_{0}^{t}\left(u^{-\alpha}\left|\varphi_{\lambda}^{*}(u \sqrt{L})(f-\rho)\right|\right)^{q} \frac{d u}{u} d \mu \\
& \lesssim \frac{V(x, t)}{w(B(x, t))^{2}} \int_{B(x, t)} \sum_{\nu \geqslant v_{0}} \int_{2^{-v-1}}^{2^{-\nu}}\left(u^{-\alpha}\left|\varphi_{\lambda}^{*}(u \sqrt{L})(f-\rho)\right|\right)^{q} \frac{d u}{u} d \mu \\
& \lesssim \frac{V(x, t)}{w(B(x, t))^{2}} \int_{B(x, t)} \sum_{\nu \geqslant v_{0}} \sum_{j \in \mathbb{Z}} 2^{-q(2 m-\alpha)|v-j|}\left(2^{j \alpha}\left|\psi_{j, \lambda}^{*}(\sqrt{L}) f\right|\right)^{q} d \mu \\
& \lesssim \frac{V(x, t)}{w(B(x, t))^{2}} \int_{B(x, t)} \sum_{\nu \geqslant v_{0}} \sum_{j \geqslant v_{0}} \cdots+\frac{V(x, t)}{w(B(x, t))^{2}} \int_{B(x, t)} \sum_{\nu \geqslant v_{0}} \sum_{j<v_{0}} \cdots \\
& =: I_{1}+I_{2} .
\end{aligned}
$$

Assume $w \in A_{1}$ and $\lambda>n / q+2 n / q$. Choose $p>1$ such that $\lambda>n p / q+2 n p^{2} / q$. Then, since $w \in A_{p}$, using Lemma 3.19, we deduce that

$$
I_{1} \lesssim\|f\|_{\dot{F}_{\infty, q, w}^{\alpha, L}}^{q}
$$

For the term $I_{2}$, we have

$$
\begin{aligned}
I_{2}= & \frac{V(x, t)}{w(B(x, t))^{2}} \int_{B(x, t)} \sum_{v \geqslant \nu_{0}} 2^{-q(2 m-\alpha)\left(v-\nu_{0}\right)} \sum_{j<\nu_{0}} 2^{-q(2 m-\alpha)\left(\nu_{0}-j\right)} \\
& \times\left(2^{j \alpha}\left|\psi_{j, \lambda}^{*}(\sqrt{L}) f\right|\right)^{q} d \mu \\
\lesssim & \sum_{j<\nu_{0}} 2^{-q(2 m-\alpha)\left(\nu_{0}-j\right)} \frac{V(x, t)}{w(B(x, t))^{2}} \int_{B(x, t)}\left(2^{j \alpha}\left|\psi_{j, \lambda}^{*}(\sqrt{L}) f\right|\right)^{q} d \mu .
\end{aligned}
$$


We now claim that

$$
\begin{aligned}
& \frac{1}{V(x, t)} \int_{B(x, t)}\left(2^{j \alpha}\left|\psi_{j, \lambda}^{*}(\sqrt{L}) f\right|\right)^{q} d \mu(y) \\
& \quad \lesssim \frac{1}{V\left(x, 2^{v_{0}-j} t\right)} \int_{B\left(x, 2^{v_{0}-j} t\right)}\left(2^{j \alpha}\left|\psi_{j, \lambda}^{*}(\sqrt{L}) f\right|\right)^{q} d \mu(y) .
\end{aligned}
$$

Indeed, for any $y \in B(x, t)$ and $y^{\prime} \in B\left(x, 2^{v_{0}-j} t\right)$ with $j<v_{0}$, we have $d(y$, $\left.y^{\prime}\right) \lesssim 2^{-j}$. Hence,

$$
\begin{aligned}
\psi_{j, \lambda}^{*}(\sqrt{L}) f(y) & =\sup _{z \in X} \frac{\left|\psi_{j, \lambda}(\sqrt{L}) f(z)\right|}{\left(1+2^{j} d(y, z)\right)^{\lambda}} \sim \sup _{z \in X} \frac{\left|\psi_{j, \lambda}(\sqrt{L}) f(z)\right|}{\left(1+2^{j} d(y, z)+2^{j} d\left(y, y^{\prime}\right)\right)^{\lambda}} \\
& \leqslant \sup _{z \in X} \frac{\left|\psi_{j, \lambda}(\sqrt{L}) f(z)\right|}{\left(1+2^{j} d\left(z, y^{\prime}\right)\right)^{\lambda}} \\
& =\psi_{j, \lambda}^{*}(\sqrt{L}) f\left(y^{\prime}\right)
\end{aligned}
$$

so that $\sup _{y \in B(x, t)} \psi_{j, \lambda}^{*}(\sqrt{L}) f(y) \lesssim \inf _{y^{\prime} \in B\left(x, 2^{v_{0}-j} t\right)} \psi_{j, \lambda}^{*}(\sqrt{L}) f\left(y^{\prime}\right)$. Thus, (79) follows.

Using (79) and Lemma 3.19, for each $j<v_{0}$, we have

$$
\begin{aligned}
& \frac{V(x, t)}{w(B(x, t))^{2}} \int_{B(x, t)}\left(2^{j \alpha}\left|\psi_{j, \lambda}^{*}(\sqrt{L}) f\right|\right)^{q} d \mu \\
& \quad \lesssim \frac{V(x, t)^{2}}{w(B(x, t))^{2}} \frac{1}{V\left(x, 2^{v_{0}-j} t\right)} \int_{B\left(x, 2^{v_{0}-j} t\right)}\left(2^{j \alpha}\left|\psi_{j, \lambda}^{*}(\sqrt{L}) f\right|\right)^{q} d \mu \\
& \quad \lesssim \frac{V\left(x, 2^{v_{0}-j} t\right)}{w\left(B\left(x, 2^{v_{0}-j} t\right)\right)^{2}} \int_{B\left(x, 2^{v_{0}-j} t\right)}\left(2^{j \alpha}\left|\psi_{j, \lambda}^{*}(\sqrt{L}) f\right|\right)^{q} d \mu \\
& \quad \lesssim\|f\|_{\dot{F}_{\infty, q, q, w}^{\alpha, L}}^{q},
\end{aligned}
$$

where in the second inequality, we used the fact that

$$
\frac{V(x, t)}{w(B(x, t))} \lesssim \frac{V\left(x, 2^{v_{0}-j} t\right)}{w\left(B\left(x, 2^{v_{0}-j} t\right)\right)}
$$

which is a consequence of the $A_{1}$-condition (see Lemma 2.1). Inserting (80) into (78) yields

$$
I_{2} \lesssim\|f\|_{\dot{F}_{\infty, q, w}^{\alpha, L}}^{q} .
$$

The above estimate for $I_{2}$ and (77) imply (74).

If $q>1$, using Young's inequality and a similar argument, we also obtain that

$$
\frac{V(x, t)}{w(B(x, t))^{2}} \int_{B(x, t)} \int_{0}^{t}\left(u^{-\alpha}\left|\varphi_{\lambda}^{*}(u \sqrt{L})(f-\rho)\right|\right)^{q} \frac{d u}{u} d \mu(x) \lesssim\|f\|_{\dot{F}_{\infty, q, w}^{\alpha, L}}^{q} .
$$

This completes the proof of (74) in all cases. 
It remains to prove $(75)$. We first show that

$$
\|f\|_{\dot{F}_{\infty, q, w}^{\alpha, L}} \lesssim K_{\lambda, \alpha, q, L}^{*}(f) .
$$

To do this, let $\psi$ be a partition of unity. Assume that $t \in\left(2^{-v+1}, 2^{-v+2}\right]$ with $v \in \mathbb{Z}$. By Proposition 2.18, for any $\lambda>0$,

$$
\begin{aligned}
\sum_{j \geqslant v}\left(2^{j \alpha}\left|\psi_{j}(\sqrt{L}) f(x)\right|\right)^{q} & \lesssim \sum_{j \geqslant \nu} \int_{2^{-j-2}}^{2^{-j+2}}\left|t^{-\alpha} \varphi_{\lambda}^{*}(s \sqrt{L}) f(x)\right|^{q} \frac{d s}{s} \\
& \lesssim \int_{0}^{2^{-v+2}}\left|t^{-\alpha} \varphi_{\lambda}^{*}(s \sqrt{L}) f(x)\right|^{q} \frac{d s}{s} .
\end{aligned}
$$

This clearly implies (81).

Therefore, to complete the proof of (75), it suffices to prove that

$$
K_{\lambda, \alpha, q, L}^{*}(f) \lesssim K_{\alpha, q, L}(f) .
$$

To this end, fix $A>\lambda+\alpha$. Then for each $j \geqslant v$ and $t \in[1,2]$, recall that

$$
\varphi_{\lambda}^{* *}\left(2^{-j} t \sqrt{L}\right)(L) f(x)=\sup _{k \geqslant j} \sup _{y \in X} 2^{-(k-j) A} \frac{\left|\varphi\left(2^{-k} t \sqrt{L}\right) f(y)\right|}{\left(1+2^{j} d(x, y)\right)^{\lambda}} .
$$

Applying (58) with $r=q / p$ gives

$$
\left|\varphi_{\lambda}^{*}\left(2^{-j} t \sqrt{L}\right) f(x)\right|^{r} \lesssim \sum_{k \geqslant j} 2^{-(k-j)(A-\lambda) r} \int_{X} \frac{\left|\varphi\left(2^{-k} t \sqrt{L}\right) f(z)\right|^{r}}{V\left(z, 2^{-k}\right)\left(1+2^{k} d(x, z)\right)^{\lambda r}} d \mu(z) .
$$

Since $p=q / r>1$, it follows that

$$
\begin{aligned}
& {\left[\int_{1}^{2}\left|\varphi_{\lambda}^{*}\left(2^{-j} t \sqrt{L}\right) f(x)\right|^{q} \frac{d t}{t}\right]^{r / q}} \\
& \quad \lesssim \sum_{k \geqslant j} 2^{-(k-j)(A-\lambda) r}\left\{\int_{1}^{2}\left[\int_{X} \frac{\left|\varphi\left(2^{-k} t \sqrt{L}\right) f(z)\right|^{r}}{V\left(z, 2^{-k}\right)\left(1+2^{k} d(x, z)\right)^{\lambda r}} d \mu(z)\right]^{q / r} \frac{d t}{t}\right\}^{r / q} .
\end{aligned}
$$

Fix a ball $Q$ and let $x \in Q$. The argument in the proof of Lemma 3.19 then shows that

$$
\left[\int_{X} \frac{\left|\varphi\left(2^{-k} t \sqrt{L}\right) f(z)\right|^{r}}{V\left(z, 2^{-k}\right)\left(1+2^{k} d(x, z)\right)^{\lambda r}} d \mu(z)\right]^{q / r}
$$




$$
\begin{aligned}
\lesssim & {\left[\mathcal{M}_{r}\left(\left|\varphi\left(2^{-k} t \sqrt{L}\right) f\right| \chi_{4 Q}\right)(x)\right]^{q} } \\
& +\sum_{\ell=3}^{\infty} \frac{2^{-\ell(\lambda q / p-n)}}{V\left(2^{\ell} Q\right)} \int_{S_{\ell}(Q)}\left|\varphi\left(2^{-k} t \sqrt{L}\right) f(z)\right|^{q} d \mu(z) .
\end{aligned}
$$

Inserting this into (82), we obtain

$$
\begin{aligned}
& {\left[\int_{1}^{2}\left|\varphi_{\lambda}^{*}\left(2^{-j} t \sqrt{L}\right) f(x)\right|^{q} \frac{d t}{t}\right]^{r / q}} \\
& \quad \lesssim \sum_{k \geqslant j} 2^{-(k-j)(A-\lambda) r}\left\{\int_{1}^{2}\left[\mathcal{M}_{r}\left(\left|\varphi\left(2^{-k} t \sqrt{L}\right) f\right| \chi_{4 Q}\right)(x)\right]^{q} \frac{d t}{t}\right\}^{r / q} \\
& \quad+\sum_{k \geqslant j} 2^{-(k-j)(A-\lambda) r} \\
& \quad \times\left\{\int_{1}^{2} \sum_{\ell=3}^{\infty} \frac{2^{-\ell(\lambda q / p-n)}}{V\left(2^{\ell} Q\right)} \int_{S_{\ell}(Q)}\left|\varphi\left(2^{-k} t \sqrt{L}\right) f(z)\right|^{q} d \mu(z) \frac{d t}{t}\right\}^{r / q} .
\end{aligned}
$$

This implies that, for any $v \in \mathbb{Z}$,

$$
\begin{aligned}
& \sum_{j \geqslant v} \int_{2^{-j}}^{2^{-j+1}}\left(2^{j \alpha}\left|\varphi_{\lambda}^{*}(t \sqrt{L}) f(x)\right|\right)^{q} \frac{d t}{t} \\
& \lesssim \sum_{j \geqslant v}\left(\sum_{k \geqslant j} 2^{-(k-j)(A-\lambda-\alpha) r}\left\{\int_{2^{-k}}^{2^{-k+1}} 2^{k \alpha q}\left[\mathcal{M}_{r}\left(|\varphi(t \sqrt{L}) f| \chi_{4 Q}\right)(x)\right]^{q} \frac{d t}{t}\right\}^{r / q}\right)^{q / r} \\
& \quad+\sum_{j \geqslant v}\left(\sum _ { k \geqslant j } 2 ^ { - ( k - j ) ( A - \lambda - \alpha ) r } \left\{\int_{2^{-k}}^{2^{-k+1}} \sum_{\ell=3}^{\infty} \frac{2^{-\ell(\lambda q / p-n)}}{V\left(2^{\ell} Q\right)} \int_{S_{\ell}(Q)}\right.\right. \\
& \left.\left.\quad \times\left(2^{k \alpha}|\varphi(t \sqrt{L}) f(z)|\right)^{q} d \mu(z) \frac{d t}{t}\right\}^{r / q}\right)^{q / r} \cdot
\end{aligned}
$$

We now use Young's inequality to get the bound:

$$
\begin{aligned}
& \sum_{j \geqslant \nu} \int_{2^{-j}}^{2^{-j+1}}\left(t^{-\alpha}\left|\varphi_{\lambda}^{*}(t \sqrt{L}) f(x)\right|\right)^{q} \frac{d t}{t} \\
& \quad \lesssim \sum_{k \geqslant v} \int_{2^{-k}}^{2^{-k+1}} t^{-\alpha q}\left[\mathcal{M}_{r}\left(|\varphi(t \sqrt{L}) f| \chi_{4 Q}\right)(x)\right]^{q} \frac{d t}{t} \\
& \quad+\sum_{k \geqslant v} \int_{2^{-k}}^{2^{-k+1}} \sum_{\ell=3}^{\infty} \frac{2^{-\ell(\lambda q / p-n)}}{V\left(2^{\ell} Q\right)} \int_{S_{\ell}(Q)}\left(t^{-\alpha}|\varphi(t \sqrt{L}) f(z)|\right)^{q} d \mu(z) \frac{d t}{t}
\end{aligned}
$$


It follows that

$$
\begin{aligned}
\int_{0}^{2^{-v+1}} & \left(t^{-\alpha}\left|\varphi_{\lambda}^{*}(t \sqrt{L}) f(x)\right|\right)^{q} \frac{d t}{t} \\
\lesssim & \int_{0}^{2^{-v+1}} t^{-\alpha q}\left[\mathcal{M}_{r}\left(|\varphi(t \sqrt{L}) f| \chi_{4 Q}\right)(x)\right]^{q} \frac{d t}{t} \\
& +\int_{0}^{2^{-v+1}} \sum_{\ell=3}^{\infty} \frac{2^{-\ell(\lambda q / p-n)}}{V\left(2^{\ell} Q\right)} \int_{S_{\ell}(Q)}\left(t^{-\alpha}|\varphi(t \sqrt{L}) f(z)|\right)^{q} d \mu(z) \frac{d t}{t} .
\end{aligned}
$$

Using the above estimate and arguing similarly to the proof of Lemma 3.19, we deduce that $K_{\lambda, \alpha, q, L}^{*}(f) \lesssim K_{\alpha, q, L}(f)$. This completes the proof of (75), and, hence, the theorem follows.

REMARK 3.22. It is natural to question if the estimate (74) holds true for $w \in A_{\infty}$. We note that when $L=-\Delta$ is the Laplacian on $\mathbb{R}^{n}$, we show in Theorem 5.6 that the classical weighted BMO space $\mathrm{BMO}_{w}\left(\mathbb{R}^{n}\right)$ coincides with $\dot{F}_{\infty, 2, w}^{0,-\Delta}\left(\mathbb{R}^{n}\right)$ for $w \in A_{1} \cap \mathrm{RH}_{2}$. In this case, the estimate (74) is known as the Carleson measure condition and, to the best of our knowledge, the problem of obtaining the estimate (74) on the classical weighted BMO space with all $w \in A_{\infty}$ is still open. In this sense, the restriction $w \in A_{1}$ is reasonable.

If we replace the definition of $\|f\|_{\dot{F}_{\alpha, q, w}^{\alpha, \psi, L}}$ in Definition 3.16 by the following quantity

$$
\sup _{Q: \text { balls }}\left(\frac{1}{w(Q)} \int_{Q} \sum_{j \geqslant-\log _{2} r_{Q}}^{\infty}\left(2^{j \alpha}\left|\psi_{j}(\sqrt{L}) f(x)\right|\right)^{q} w(x) d \mu(x)\right)^{1 / q},
$$

then by a similar argument, we can show that (74) holds true for $w \in A_{\infty}$. However, one of the main drawbacks of this definition is that when $L=-\Delta$, we have $\dot{F}_{\infty, 2, w}^{0,-}\left(\mathbb{R}^{n}\right) \equiv \operatorname{BMO}\left(\mathbb{R}^{n}\right)$ for every $w \in A_{\infty}$. See, for example, [13].

We have the following results as a direct consequence of Theorem 3.21.

Corollary 3.23. Let $0<q \leqslant \infty, \alpha \in \mathbb{R}, m>\alpha / 2$ and $w \in A_{p}, 1 \leqslant p<\infty$. Assume $\lambda>n p / q+2 n p^{2} / q$. Let $\Psi_{m, t}(L)=\left(t^{2} L\right)^{m} e^{-t^{2} L}$ and

$$
\Psi_{m, t, \lambda}^{*}(L) f(x)=\sup _{y \in X} \frac{\left|\Psi_{m, t}(L) f(y)\right|}{(1+d(x, y) / t)^{\lambda}} .
$$

(i) If $p=1$, then for each $f \in \mathcal{S}^{\prime}$, there exists $\rho \in \mathscr{P}$ so that

$$
\begin{aligned}
& \sup _{x \in X, t>0}\left(\frac{V(x, t)}{w(B(x, t))^{2}} \int_{B(x, t)} \int_{0}^{t}\left(s^{-\alpha}\left|\Psi_{m, s, \lambda}^{*}(L)(f-\rho)(y)\right|\right)^{q} \frac{d s}{s} d y\right)^{1 / q} \\
& \quad \lesssim\|f\|_{\dot{F}_{\infty, q, w}^{\alpha, L}} .
\end{aligned}
$$


(ii) If $p>1$, then

$$
\|f\|_{\dot{F}_{\infty, q, w}^{\alpha, L}} \lesssim \sup _{x \in X, t>0}\left(\frac{V(x, t)}{w(B(x, t))^{2}} \int_{B(x, t)} \int_{0}^{t}\left(s^{-\alpha}\left|\Psi_{m, s}(L) f(y)\right|\right)^{q} \frac{d s}{s} d y\right)^{1 / q}
$$

for every $f \in \mathcal{S}^{\prime}$.

Moreover, the distribution $\rho$ can be removed if $f \in L^{2}(X)$.

\section{Atomic decompositions}

We now prove atomic decomposition theorems for our new Besov and TriebelLizorkin spaces. We first introduce the definition of weighted atoms related to $L$.

Definition 4.1. Let $0<p \leqslant \infty, M \in \mathbb{N}_{+}$and $w \in A_{\infty}$. A function $a$ is said to be an $(L, M, p, w)$ atom if there exists a dyadic cube $Q \in \mathcal{D}$ so that:

(i) $a=L^{M} b$ with $b \in D\left(L^{M}\right)$, where $D\left(L^{M}\right)$ is the domain of $L^{M}$;

(ii) $\operatorname{supp} L^{k} b \subset 3 B_{Q}, k=0, \ldots, 2 M$;

(iii) $\left|L^{k} b(x)\right| \leqslant \ell(Q)^{2(M-k)} w(Q)^{-1 / p}, k=0, \ldots, 2 M$;

where $B_{Q}$ is a ball associated with $Q$ defined in Remark 2.4.

4.1. Atomic decompositions for Besov spaces $\dot{B}_{p, q, w}^{\alpha, L}$. Our first main result in this section is the following theorem in which we show that each function in $\dot{B}_{p, q, w}^{\alpha, L}$ can be characterized in terms of atomic decompositions.

Theorem 4.2. Let $\alpha \in \mathbb{R}, 0<p, q \leqslant \infty, M \in \mathbb{N}_{+}$and $w \in A_{\infty}$. Assume $f \in \dot{B}_{p, q, w}^{\alpha, L}$. Then there exist a sequence of $(L, M, p, w)$ atoms $\left\{a_{Q}\right\}_{Q \in \mathcal{D}_{v}, v \in \mathbb{Z}}$ and a sequence of coefficients $\left\{s_{Q}\right\}_{Q \in \mathcal{D}_{v}, v \in \mathbb{Z}}$ so that

$$
f=\sum_{v \in \mathbb{Z}} \sum_{Q \in \mathcal{D}_{v}} s_{Q} a_{Q} \quad \text { in } \mathcal{S}_{\infty}^{\prime}
$$

Moreover,

$$
\left[\sum_{\nu \in \mathbb{Z}} 2^{v \alpha q}\left(\sum_{Q \in \mathcal{D}_{v}}\left|s_{Q}\right|^{p}\right)^{q / p}\right]^{1 / q} \lesssim\|f\|_{\dot{B}_{p, q, w}^{\alpha, L}}
$$


Proof. Let $\psi$ be a partition of unity and $\Phi$ be a function as in Lemma 2.5. Due to Proposition 2.11, for $f \in \mathcal{S}_{\infty}^{\prime}$, we have

$$
f=c \int_{0}^{\infty} \psi(t \sqrt{L}) \Phi(t \sqrt{L}) f \frac{d t}{t}
$$

in $\mathcal{S}_{\infty}^{\prime}$, where $c=\left[\int_{0}^{\infty} \psi(\xi) \Phi(\xi) \frac{d \xi}{\xi}\right]^{-1}$. As a consequence, by Lemma 2.3, we have

$$
\begin{aligned}
f & =c \sum_{\nu \in \mathbb{Z}} \int_{2^{-v-1}}^{2^{-v}}\left(t^{2} L\right)^{M} \Phi(t \sqrt{L})\left[\psi_{M}(t \sqrt{L}) f\right] \frac{d t}{t} \\
& =c \sum_{\nu \in \mathbb{Z}} \sum_{Q \in \mathcal{D}_{v}} \int_{2^{-v-1}}^{2^{-v}}\left(t^{2} L\right)^{M} \Phi(t \sqrt{L})\left[\psi_{M}(t \sqrt{L}) f \cdot \chi_{Q}\right] \frac{d t}{t},
\end{aligned}
$$

where $\psi_{M}(\xi)=\xi^{-2 M} \psi(\xi)$.

For each $v \in \mathbb{Z}$ and $Q \in \mathcal{D}_{v}$, we set

$$
s_{Q}=w(Q)^{1 / p} \sup _{y \in Q} \int_{2^{-\nu-1}}^{2^{-\nu}}\left|\psi_{M}(t \sqrt{L}) f(y)\right| \frac{d t}{t},
$$

and $a_{Q}=L^{M} b_{Q}$, where

$$
b_{Q}=\frac{1}{s_{Q}} \int_{2^{-v-1}}^{2^{-v}} t^{2 M} \Phi(t \sqrt{L})\left[\psi_{M}(t \sqrt{L}) f \cdot \chi_{Q}\right] \frac{d t}{t} .
$$

Obviously, we deduce from (84) that

$$
f=\sum_{v \in \mathbb{Z}} \sum_{Q \in \mathcal{D}_{v}} s_{Q} a_{Q} \quad \text { in } \mathcal{S}_{\infty}^{\prime}
$$

For $k=0, \ldots, 2 M$, we have

$$
\begin{aligned}
L^{k} b_{Q}(x) & =\frac{1}{s_{Q}} \int_{2^{-v-1}}^{2^{-v}} t^{2(M-k)}\left(t^{2} L\right)^{k} \Phi(t \sqrt{L})\left[\psi_{M}(t \sqrt{L}) f \cdot \chi_{Q}\right] \frac{d t}{t} \\
& =\frac{1}{s_{Q}} \int_{2^{-v-1}}^{2^{-v}} \int_{Q} t^{2(M-k)} K_{\left(t^{2} L\right)^{k} \Phi(t \sqrt{L})}(x, y) \psi_{M}(t \sqrt{L}) f(y) d \mu(y) \frac{d t}{t} .
\end{aligned}
$$

Using the finite propagation property in Lemma 2.5, we can see that

$$
\operatorname{supp} L^{k} b \subset 3 B_{Q},
$$


and

$$
\begin{aligned}
\left|L^{k} b_{Q}(x)\right| & \lesssim \frac{2^{-2 v(M-k)}}{s_{Q}} \int_{2^{-v-1}}^{2^{-v}} \int_{Q} \frac{1}{V\left(y, 2^{-v}\right)}\left|\psi_{M}(t \sqrt{L}) f(y)\right| d \mu(y) \frac{d t}{t} \\
& \lesssim 2^{-v(2 M-2 k)} w(Q)^{-1 / p}
\end{aligned}
$$

It follows that $a_{Q}$ is (a multiple of) an $(L, M, p, w)$ atom. We now prove that

$$
\left[\sum_{v \in \mathbb{Z}} 2^{v \alpha q}\left(\sum_{Q \in \mathcal{D}_{v}}\left|s_{Q}\right|^{p}\right)^{q / p}\right]^{1 / q} \sim\|f\|_{\dot{B}_{p, q, w}^{\alpha, L}} .
$$

Indeed, for any $\lambda>0$, we note that

$$
\begin{aligned}
s_{Q} & \sim w(Q)^{1 / p} \sup _{y \in Q} \int_{2^{-v-1}}^{2^{-v}}\left|\psi_{M}(t \sqrt{L}) f(y)\right| \frac{d t}{t} \\
& \leqslant\left[\int_{Q}\left|F_{M, \lambda}^{*}(\sqrt{L}) f(x)\right|^{p} w(x) d \mu(x)\right]^{1 / p},
\end{aligned}
$$

where

$$
F_{M, \lambda}^{*}(\sqrt{L}) f(x)=\sup _{y \in X} \frac{\int_{2^{-v-1}}^{2^{-v}}\left|\psi_{M}(t \sqrt{L}) f(y)\right| \frac{d t}{t}}{\left(1+2^{v} d(x, y)\right)^{\lambda}} .
$$

As a consequence,

$$
\sum_{Q \in \mathcal{D}_{v}}\left|s_{Q}\right|^{p} \leqslant \int_{X}\left|F_{M, s, \lambda}^{*}(\sqrt{L}) f(x)\right|^{p} w(x) d \mu(x) .
$$

On the other hand, fixing a $m>\alpha / 2$ and arguing similarly to (55), we obtain

$$
\left|F_{M, \lambda}^{*}(\sqrt{L}) f\right| \lesssim \sum_{j \in \mathbb{Z}} 2^{-2 m|v-j|} \psi_{j, \lambda}^{*}(\sqrt{L}) f .
$$

Therefore,

$$
\begin{aligned}
& {\left[\sum_{v \in \mathbb{Z}} 2^{v \alpha q}\left(\sum_{Q \in \mathcal{D}_{v}}\left|s_{Q}\right|^{p}\right)^{q / p}\right]^{1 / q}} \\
& \leqslant\left[\sum_{v \in \mathbb{Z}} 2^{v \alpha q}\left(\left\|\sum_{j \in \mathbb{Z}} 2^{-2 m|v-j|} \psi_{j, \lambda}^{*}(\sqrt{L}) f\right\|_{p, w}\right)^{q}\right]^{1 / q} \\
& \leqslant\left[\sum_{v \in \mathbb{Z}}\left(\left\|\sum_{j \in \mathbb{Z}} 2^{-(2 m-\alpha)|v-j|} 2^{j \alpha} \psi_{j, \lambda}^{*}(\sqrt{L}) f\right\|_{p, w}\right)^{q}\right]^{1 / q} .
\end{aligned}
$$


Case 1: $p \geqslant 1$. We have

$$
\begin{aligned}
& {\left[\sum_{v \in \mathbb{Z}} 2^{v \alpha q}\left(\sum_{Q \in \mathcal{D}_{v}}\left|s_{Q}\right|^{p}\right)^{q / p}\right]^{1 / q}} \\
& \leqslant\left[\sum_{\nu \in \mathbb{Z}}\left(\sum_{j \in \mathbb{Z}} 2^{-(2 m-\alpha)|v-j|} 2^{j \alpha}\left\|\psi_{j, \lambda}^{*}(\sqrt{L}) f\right\|_{p, w}\right)^{q}\right]^{1 / q} .
\end{aligned}
$$

At this stage, if $q \geqslant 1$, we then use Young's inequality and Proposition 3.3 to further imply

$$
\left[\sum_{v \in \mathbb{Z}} 2^{\nu \alpha q}\left(\sum_{Q \in \mathcal{D}_{v}}\left|s_{Q}\right|^{p}\right)^{q / p}\right]^{1 / q} \leqslant\left[\sum_{j \in \mathbb{Z}}\left(2^{j \alpha}\left\|\psi_{j, \lambda}^{*}(\sqrt{L}) f\right\|_{p, w}\right)^{q}\right]^{1 / q} \sim\|f\|_{\dot{B}_{p, q, w}^{\alpha, L}} .
$$

Otherwise, if $0<q<1$, we then have

$$
\begin{aligned}
& {\left[\sum_{v \in \mathbb{Z}} 2^{v \alpha q}\left(\sum_{Q \in \mathcal{D}_{v}}\left|s_{Q}\right|^{p}\right)^{q / p}\right]^{1 / q}} \\
& \leqslant\left[\sum_{v \in \mathbb{Z}} \sum_{j \in \mathbb{Z}}\left(2^{-(2 m-\alpha)|\nu-j|} 2^{j \alpha}\left\|\psi_{j, \lambda}^{*}(\sqrt{L}) f\right\|_{p, w}\right)^{q}\right]^{1 / q} \\
& \leqslant\left[\sum_{j \in \mathbb{Z}}\left(2^{j \alpha}\left\|\psi_{j, \lambda}^{*}(\sqrt{L}) f\right\|_{p, w}\right)^{q}\right]^{1 / q} \sim\|f\|_{\dot{B}_{p, q, w}^{\alpha, L}}
\end{aligned}
$$

where, in the last line, we use Proposition 3.3.

Case 2: $0<p<1$. From (86), we obtain

$$
\begin{aligned}
& {\left[\sum_{v \in \mathbb{Z}} 2^{v \alpha q}\left(\sum_{Q \in \mathcal{D}_{v}}\left|s_{Q}\right|^{p}\right)^{q / p}\right]^{1 / q}} \\
& \leqslant\left[\sum_{v \in \mathbb{Z}}\left(\sum_{j \in \mathbb{Z}} 2^{-p(2 m-\alpha)|v-j|} 2^{j s p}\left\|\psi_{j, \lambda}^{*}(\sqrt{L}) f\right\|_{p, w}^{p}\right)^{q / p}\right]^{1 / q} .
\end{aligned}
$$

If $q / p \geqslant 1$, we then use Young's inequality and Proposition 3.3 to get the estimate

$$
\left[\sum_{v \in \mathbb{Z}} 2^{v \alpha q}\left(\sum_{Q \in \mathcal{D}_{v}}\left|s_{Q}\right|^{p}\right)^{q / p}\right]^{1 / q} \leqslant\left[\sum_{j \in \mathbb{Z}}\left(2^{j \alpha}\left\|\psi_{j, \lambda}^{*}(\sqrt{L}) f\right\|_{p, w}\right)^{q}\right]^{1 / q} \sim\|f\|_{\dot{B}_{p, q, w}^{\alpha, L}} .
$$

Otherwise, if $0<q / p<1$, we then have

$$
\left[\sum_{v \in \mathbb{Z}} 2^{v \alpha q}\left(\sum_{Q \in \mathcal{D}_{v}}\left|s_{Q}\right|^{p}\right)^{q / p}\right]^{1 / q}
$$




$$
\begin{aligned}
& \leqslant\left[\sum_{v \in \mathbb{Z}} \sum_{j \in \mathbb{Z}}\left(2^{-(2 m-\alpha)|v-j|} 2^{j \alpha}\left\|\psi_{j, \lambda}^{*}(\sqrt{L}) f\right\|_{p, w}\right)^{q}\right]^{1 / q} \\
& \leqslant\left[\sum_{j \in \mathbb{Z}}\left(2^{j \alpha}\left\|\psi_{j, \lambda}^{*}(\sqrt{L}) f\right\|_{p, w}\right)^{q}\right]^{1 / q} \sim\|f\|_{\dot{B}_{p, q, w}^{\alpha, L}},
\end{aligned}
$$

where, in the last line, we use Proposition 3.3.

This completes the proof.

Conversely, each atomic decomposition with suitable coefficients belongs to the spaces $\dot{B}_{p, q, w}^{\alpha, L}$.

TheOREM 4.3. Let $\alpha \in \mathbb{R}, 0<p, q \leqslant \infty$ and $w \in A_{\infty}$. Assume that

$$
f=\sum_{v \in \mathbb{Z}} \sum_{Q \in \mathcal{D}_{v}} s_{Q} a_{Q} \quad \text { in } \mathcal{S}_{\infty}^{\prime}
$$

where $\left\{a_{Q}\right\}_{Q \in \mathcal{D}_{v}, v \in \mathbb{Z}}$ is a sequence of $(L, M, p, w)$ atoms and $\left\{s_{Q}\right\}_{Q \in \mathcal{D}_{v}, v \in \mathbb{Z}}$ is a sequence of coefficients satisfying

$$
\left[\sum_{v \in \mathbb{Z}} 2^{v \alpha q}\left(\sum_{Q \in \mathcal{D}_{v}}\left|s_{Q}\right|^{p}\right)^{q / p}\right]^{1 / q}<\infty
$$

Then $f \in \dot{B}_{p, q, w}^{\alpha, L}$ and

$$
\|f\|_{\dot{B}_{p, q, w}^{\alpha, L}} \lesssim\left[\sum_{v \in \mathbb{Z}} 2^{v \alpha q}\left(\sum_{Q \in \mathcal{D}_{v}}\left|s_{Q}\right|^{p}\right)^{q / p}\right]^{1 / q}
$$

provided $M>\frac{n}{2}+\frac{1}{2} \max \left\{\alpha, \frac{n q_{w}}{1 \wedge p \wedge q}-\alpha\right\}$.

Before coming to the proof of Theorem 4.3, we need the following technical results.

LemMA 4.4. Let $w \in A_{\tilde{q}}, N>n, \kappa \in[0,1]$ and $\eta, v \in \mathbb{Z}, v \geqslant \eta$. Assume that $\left\{f_{Q}\right\}_{Q \in \mathcal{D}_{v}}$ is a sequence of functions satisfying

$$
\left|f_{Q}(x)\right| \lesssim\left(\frac{V(Q)}{V\left(x_{Q}, 2^{-\eta}\right)}\right)^{\kappa}\left(1+\frac{d\left(x, x_{Q}\right)}{2^{-\eta}}\right)^{-N}
$$

Then for $\frac{n \tilde{q}}{N}<r \leqslant 1$ and a sequence of numbers $\left\{s_{Q}\right\}_{Q \in \mathcal{D}_{v}}$, we have

$$
\sum_{Q \in \mathcal{D}_{v}}\left|s_{Q}\right|\left|f_{Q}(x)\right| \lesssim 2^{n(\nu-\eta)(\tilde{q} / r-\kappa)} \mathcal{M}_{w, r}\left(\sum_{Q \in \mathcal{D}_{v}}\left|s_{Q}\right| \chi_{Q}\right)(x) .
$$


Proof. In the particular case when $\kappa=0, w \equiv 1$ and $X=\mathbb{R}^{n}$, this lemma was proved in [35]. We adapt some of this argument to our present situation.

Fix $x \in X$. We set

$$
\mathcal{B}_{0}=\left\{Q \in \mathcal{D}_{v}: d\left(x, x_{Q}\right) \leqslant 2^{-\eta}\right\}, \quad Q_{0}=\bigcup_{Q \in \mathcal{B}_{0}} Q
$$

and

$$
\mathcal{B}_{k}=\left\{Q \in \mathcal{D}_{v}: 2^{k-\eta-1}<d\left(x, x_{Q}\right) \leqslant 2^{k-\eta}\right\}, \quad Q_{k}=\bigcup_{j: j \leqslant k} \bigcup_{Q \in \mathcal{B}_{j}} Q, \quad k \in \mathbb{N}_{+} .
$$

Then we write

$$
\begin{aligned}
\sum_{Q \in \mathcal{D}_{v}}\left|s_{Q}\right|\left|f_{Q}(x)\right| & =\sum_{k \in \mathbb{N}} \sum_{Q \in \mathcal{B}_{k}}\left|s_{Q}\right|\left|f_{Q}(x)\right| \\
& \leqslant \sum_{k \in \mathbb{N}} \sum_{Q \in \mathcal{B}_{k}}\left|s_{Q}\right|\left(\frac{V(Q)}{V\left(x_{Q}, 2^{-\eta}\right)}\right)^{\kappa}\left(1+\frac{d\left(x, x_{Q}\right)}{2^{-\eta}}\right)^{-N} \\
& =: \sum_{k \in \mathbb{N}} E_{k} .
\end{aligned}
$$

For each $k \in \mathbb{N}$, we have

$$
\begin{aligned}
E_{k} \lesssim & \sum_{Q \in \mathcal{B}_{k}} 2^{-k N}\left(\frac{V(Q)}{V\left(x_{Q}, 2^{-\eta}\right)}\right)^{\kappa}\left|s_{Q}\right| \leqslant 2^{-k N}\left[\sum_{Q \in \mathcal{B}_{k}}\left(\frac{V(Q)}{V\left(x_{Q}, 2^{-\eta}\right)}\right)^{\kappa r}\left|s_{Q}\right|^{r}\right]^{1 / r} \\
\lesssim & 2^{-k N}\left\{\int_{Q_{k}}\left[\sum_{Q \in \mathcal{B}_{k}}\left(\frac{V(Q)}{V\left(x_{Q}, 2^{-\eta}\right)}\right)^{\kappa} w(Q)^{-1 / r}\left|s_{Q}\right| \chi_{Q}(y)\right]^{r} w(y) d \mu(y)\right\}^{1 / r} \\
\lesssim & 2^{-k N}\left\{\frac { 1 } { w ( Q _ { k } ) } \int _ { Q _ { k } } \left[\sum_{Q \in \mathcal{B}_{k}}\left(\frac{w\left(Q_{k}\right)}{w(Q)}\right)^{1 / r}\right.\right. \\
& \left.\left.\times\left(\frac{V(Q)}{V\left(x_{Q}, 2^{-\eta}\right)}\right)^{\kappa}\left|s_{Q}\right| \chi_{Q}(y)\right]^{r} d \mu(y)\right\}^{1 / r} .
\end{aligned}
$$

It is easy to see that $V\left(Q_{k}\right) \sim V\left(x, 2^{-\eta+k}\right) \sim V\left(x_{Q}, 2^{-\eta+k}\right)$, for each $Q \in \mathcal{B}_{k}$. Therefore,

$$
\begin{aligned}
\left(\frac{w\left(Q_{k}\right)}{w(Q)}\right)^{1 / r}\left(\frac{V(Q)}{V\left(x_{Q}, 2^{-\eta}\right)}\right)^{\kappa} & \lesssim\left(\frac{V\left(Q_{k}\right)}{V(Q)}\right)^{\tilde{q} / r}\left(\frac{V(Q)}{V\left(x_{Q}, 2^{-\eta}\right)}\right)^{\kappa} \\
& =\left(\frac{V\left(Q_{k}\right)}{V\left(x_{Q}, 2^{-\eta}\right)}\right)^{\tilde{q} / r}\left(\frac{V\left(x_{Q}, 2^{-\eta}\right)}{V(Q)}\right)^{\tilde{q} / r-\kappa} \\
& \lesssim 2^{k n \tilde{q} / r} 2^{n(\nu-\eta)(\tilde{q} / r-\kappa)} .
\end{aligned}
$$


Inserting this into (87) gives

$$
\begin{aligned}
E_{k} & \lesssim 2^{-k N} 2^{k n \tilde{q} / r} 2^{n(\nu-\eta)(\tilde{q} / r-\kappa)}\left\{\frac{1}{w\left(Q_{k}\right)} \int_{Q_{k}}\left[\sum_{Q \in \mathcal{B}_{k}}\left|s_{Q}\right| \chi_{Q}(y)\right]^{r} w(y) d \mu(y)\right\}^{1 / r} \\
& \lesssim 2^{-k(N-n \tilde{q} / r)} 2^{n(\nu-\eta)(\tilde{q} / r-\kappa)} \mathcal{M}_{w, r}\left(\sum_{Q \in \mathcal{B}_{k}}\left|s_{Q}\right| \chi_{Q}\right)(x)
\end{aligned}
$$

Since $r>\frac{n \tilde{q}}{N}$, we find that

$$
\sum_{k \in \mathbb{N}} E_{k} \lesssim 2^{n(v-\eta)(\tilde{q} / r-\kappa)} \mathcal{M}_{w, r}\left(\sum_{Q \in \mathcal{D}_{v}}\left|s_{Q}\right| \chi_{Q}\right)(x)
$$

This completes our proof.

LEMMA 4.5. Let $\psi$ be a partition of unity and let $a_{Q}$ be an $(L, M, p, w)$ atom with some $Q \in \mathcal{D}_{v}$. Then for any $t>0$ and $N>0$, we have

$$
\left|\psi(t \sqrt{L}) a_{Q}(x)\right| \lesssim\left(\frac{t}{2^{-v}} \wedge \frac{2^{-v}}{t}\right)^{2 M-n} w(Q)^{-1 / p}\left(1+\frac{d\left(x, x_{Q}\right)}{2^{-v} \vee t}\right)^{-N}
$$

Proof. We now consider two cases: $t \leqslant 2^{-v}$ and $t>2^{-v}$.

Case 1: $t \leqslant 2^{-v}$. Observe that

$$
\psi(t \sqrt{L}) a_{Q}=t^{2 M} \psi_{M}(t \sqrt{L})\left(L^{M} a_{Q}\right),
$$

where $\psi_{M}(\lambda)=\lambda^{-2 M} \psi(\lambda)$.

This, along with Lemma 2.6 and the definition of the atoms, yields

$$
\begin{aligned}
\left|\psi(t \sqrt{L}) a_{Q}(x)\right| & \lesssim \int_{3 B_{Q}} \frac{t^{2 M}}{V(y, t)}\left(1+\frac{d(x, y)}{t}\right)^{-N}\left|L^{K} a_{Q}(y)\right| d \mu(y) \\
& \lesssim\left(\frac{t}{2^{-v}}\right)^{2 M} w(Q)^{-1 / p} \int_{3 B_{Q}} \frac{1}{V(y, t)}\left(1+\frac{d(x, y)}{t}\right)^{-N} d \mu(y)
\end{aligned}
$$

Note that, for $t \leqslant 2^{-v}$ and $y \in 3 B_{Q}$, we have

$$
\left(1+\frac{d(x, y)}{t}\right)^{-N} \leqslant\left(1+\frac{d(x, y)}{2^{-v}}\right)^{-N} \sim\left(1+\frac{d\left(x, x_{Q}\right)}{2^{-v}}\right)^{-N} .
$$


Therefore,

$$
\begin{aligned}
\left|\psi(t \sqrt{L}) a_{Q}(x)\right| & \lesssim\left(\frac{t}{2^{-v}}\right)^{2 M} w(Q)^{-1 / p}\left(1+\frac{d\left(x, x_{Q}\right)}{2^{-v}}\right)^{-N} \frac{V\left(3 B_{Q}\right)}{V(y, t)} \\
& \lesssim\left(\frac{t}{2^{-v}}\right)^{2 M-n} w(Q)^{-1 / p}\left(1+\frac{d\left(x, x_{Q}\right)}{2^{-\nu}}\right)^{-N},
\end{aligned}
$$

where, in the last inequality, we use (2). This leads us to (88).

Case 2: $t>2^{-v}$. We first write $a_{Q}=L^{M} b_{Q}$. Hence,

$$
\psi(t \sqrt{L}) a_{Q}=t^{-2 M} \tilde{\psi}_{M}(t \sqrt{L}) b_{Q},
$$

where $\tilde{\psi}_{M}(\lambda)=\lambda^{2 M} \psi(\lambda)$.

This, along with Lemma 2.6, implies that

$$
\begin{aligned}
\left|\psi(t \sqrt{L}) a_{Q}(x)\right| & \lesssim \int_{3 B_{Q}} \frac{t^{-2 M}}{V(y, t)}\left(1+\frac{d(x, y)}{t}\right)^{-N}\left|b_{Q}(y)\right| d \mu(y) \\
& \lesssim\left(\frac{2^{-v}}{t}\right)^{2 M} w(Q)^{-1 / p} \int_{3 B_{Q}} \frac{1}{V(y, t)}\left(1+\frac{d(x, y)}{t}\right)^{-N} d \mu(y) .
\end{aligned}
$$

Note that for $y \in 3 B_{Q}$ and $t \geqslant 2^{-v} \sim \ell(Q)$, we have

$$
\left(1+\frac{d(x, y)}{t}\right)^{-N} \sim\left(1+\frac{d\left(x, x_{Q}\right)}{t}\right)^{-N} .
$$

Hence, the above inequality simplifies into

$$
\begin{aligned}
\left|\psi(t \sqrt{L}) a_{Q}(x)\right| & \lesssim\left(\frac{2^{-v}}{t}\right)^{2 M} w(Q)^{-1 / p}\left(1+\frac{d\left(x, x_{Q}\right)}{t}\right)^{-N} \frac{V\left(3 B_{Q}\right)}{V(y, t)} \\
& \lesssim\left(\frac{2^{-v}}{t}\right)^{2 M} w(Q)^{-1 / p}\left(1+\frac{d\left(x, x_{Q}\right)}{t}\right)^{-N} .
\end{aligned}
$$

Hence, (88) follows.

We are now ready to give the proof for Theorem 4.3.

Proof of Theorem 4.3. The proof can be done by using similar arguments to those in $[34,35]$. However, for the sake of completeness, we will provide the details.

Fix $\tilde{q} \in\left(q_{w}, \infty\right)$ and $r<\min \{1, p, q\}$ so that $w \in A_{\tilde{q}}$ and $M>n / 2+n \tilde{q} / r-s$. We now fix $N>\frac{n \tilde{q}}{r}$. Let $\psi$ be a partition of unity. Since

$$
f=\sum_{v \in \mathbb{Z}} \sum_{Q \in \mathcal{D}_{v}} s_{Q} a_{Q} \quad \text { in } \mathcal{S}_{\infty}^{\prime}
$$


we then, for each $j \in \mathbb{Z}$, have

$$
\begin{aligned}
\psi_{j}(\sqrt{L}) f & =\sum_{v \in \mathbb{Z}} \sum_{Q \in \mathcal{D}_{v}} s_{Q} \psi_{j}(\sqrt{L}) a_{Q} \\
& =\sum_{v: v \geqslant j} \sum_{Q \in \mathcal{D}_{v}} s_{Q} \psi_{j}(\sqrt{L}) a_{Q}+\sum_{v: v<j} \sum_{Q \in \mathcal{D}_{v}} s_{Q} \psi_{j}(\sqrt{L}) a_{Q} .
\end{aligned}
$$

Using Lemmas 4.4 and 4.5 , we see that

$$
\begin{aligned}
\left|\psi_{j}(\sqrt{L}) f\right| \lesssim & \sum_{v \in \mathbb{Z}} \sum_{Q \in \mathcal{D}_{v}} s_{Q} \psi_{j}(\sqrt{L}) a_{Q} \\
= & \sum_{v: v \geqslant j} 2^{-(\nu-j)(2 M-n-n \tilde{q} / r)} \mathcal{M}_{w, r}\left(\sum_{Q \in \mathcal{D}_{v}}\left|s_{Q}\right| w(Q)^{-1 / p} \chi_{Q}\right) \\
& +\sum_{v: v<j} 2^{-(2 M-n)(j-v)} \mathcal{M}_{w, r}\left(\sum_{Q \in \mathcal{D}_{v}}\left|s_{Q}\right| w(Q)^{-1 / p} \chi_{Q}\right) .
\end{aligned}
$$

Therefore,

$$
\begin{aligned}
\|f\|_{\dot{B}_{p, q, w}^{\alpha, L}}:= & {\left[\sum_{j \in \mathbb{Z}}\left(2^{j \alpha}\left\|\psi_{j}(\sqrt{L}) f\right\|_{p, w}\right)^{q}\right]^{1 / q} } \\
\lesssim & {\left[\sum _ { j \in \mathbb { Z } } \left(\| \sum_{v: v \geqslant j} 2^{-(\nu-j)(2 M-n-n \tilde{q} / r+\alpha)} \mathcal{M}_{w, r}\right.\right.} \\
& \left.\left.\times\left(\sum_{Q \in \mathcal{D}_{v}} 2^{v \alpha}\left|s_{Q}\right| w(Q)^{-1 / p} \chi_{Q}\right) \|_{p, w}\right)^{q}\right]^{1 / q} \\
& +\left[\sum _ { j \in \mathbb { Z } } \left(\| \sum_{v: v<j} 2^{-(2 M-n-\alpha)(j-v)} \mathcal{M}_{w, r}\right.\right. \\
& \left.\left.\times\left(\sum_{Q \in \mathcal{D}_{v}} 2^{v \alpha}\left|s_{Q}\right| w(Q)^{-1 / p} \chi_{Q}\right) \|_{p, w}\right)^{q}\right]^{1 / q} \\
= & E_{1}+E_{2} .
\end{aligned}
$$

If $p \geqslant 1$, then we have

$$
\begin{aligned}
E_{1} \lesssim & {\left[\sum _ { j \in \mathbb { Z } } \left(\sum_{\nu: v \geqslant j} 2^{-(\nu-j)(2 M-n-n \tilde{q} / r+\alpha)}\right.\right.} \\
& \left.\left.\times\left\|\mathcal{M}_{w, r}\left(\sum_{Q \in \mathcal{D}_{v}} 2^{v \alpha}\left|s_{Q}\right| w(Q)^{-1 / p} \chi_{Q}\right)\right\|_{p, w}\right)^{q}\right]^{1 / q} .
\end{aligned}
$$


We now apply Young's inequality when $q \geqslant 1$ and the inequality $\left(\sum_{j}\left|a_{j}\right|\right)^{q} \leqslant$ $\sum_{j}\left|a_{j}\right|^{q}$ when $0<q<1$ to simplify

$$
E_{1} \lesssim\left[\sum_{\nu \in \mathbb{Z}}\left(\left\|\mathcal{M}_{w, r}\left(\sum_{Q \in \mathcal{D}_{v}} 2^{v \alpha}\left|s_{Q}\right| w(Q)^{-1 / p} \chi_{Q}\right)\right\|_{p, w}\right)^{q}\right]^{1 / q}
$$

as long as $2 M>n \tilde{q} / r-\alpha$.

On the other hand, since the maximal function $\mathcal{M}_{w, r}$ is bounded on $L_{w}^{p}(X)$ as $p>r$, we have

$$
\begin{aligned}
\left\|\mathcal{M}_{w, r}\left(\sum_{Q \in \mathcal{D}_{v}} 2^{v \alpha}\left|s_{Q}\right| w(Q)^{-1 / p} \chi_{Q}\right)\right\|_{p, w} & \lesssim\left\|\sum_{Q \in \mathcal{D}_{v}} 2^{v \alpha}\left|s_{Q}\right| w(Q)^{-1 / p} \chi_{Q}\right\|_{p, w} \\
& \sim\left(\sum_{Q \in \mathcal{D}_{v}} 2^{v \alpha p}\left|s_{Q}\right|^{p}\right)^{1 / p} .
\end{aligned}
$$

As a consequence,

$$
E_{1} \lesssim\left[\sum_{v \in \mathbb{Z}}\left(\sum_{Q \in \mathcal{D}_{v}} 2^{v \alpha p}\left|s_{Q}\right|^{p}\right)^{q / p}\right]^{1 / q}=\left[\sum_{v \in \mathbb{Z}} 2^{v \alpha q}\left(\sum_{Q \in \mathcal{D}_{v}}\left|s_{Q}\right|^{p}\right)^{q / p}\right]^{1 / q} .
$$

Similarly,

$$
E_{2} \lesssim\left[\sum_{v \in \mathbb{Z}}\left(\sum_{Q \in \mathcal{D}_{v}} 2^{v \alpha p}\left|s_{Q}\right|^{p}\right)^{q / p}\right]^{1 / q}=\left[\sum_{\nu \in \mathbb{Z}} 2^{v \alpha q}\left(\sum_{Q \in \mathcal{D}_{v}}\left|s_{Q}\right|^{p}\right)^{q / p}\right]^{1 / q} .
$$

Hence,

$$
\|f\|_{\dot{B}_{p, q, w}^{\alpha, L}} \lesssim\left[\sum_{v \in \mathbb{Z}} 2^{v \alpha q}\left(\sum_{Q \in \mathcal{D}_{v}}\left|s_{Q}\right|^{p}\right)^{q / p}\right]^{1 / q}
$$

as desired.

If $0<p<1$, then we have

$$
\begin{aligned}
E_{1} \lesssim & {\left[\sum _ { j \in \mathbb { Z } } \left(\sum_{\nu: \nu<j} 2^{-p(v-j)(2 M-n-n \tilde{q} / r+\alpha)}\right.\right.} \\
& \left.\left.\times\left\|\mathcal{M}_{w, r}\left(\sum_{Q \in \mathcal{D}_{v}} 2^{v \alpha}\left|s_{Q}\right| w(Q)^{-1 / p} \chi_{Q}\right)\right\|_{p, w}^{p}\right)^{q / p}\right]^{1 / q} .
\end{aligned}
$$

Arguing similarly to the case $p \geqslant 1$ by considering two cases $q / p \geqslant 1$ and $0<$ $q / p<1$, we come up with

$$
E_{1} \lesssim\left[\sum_{v \in \mathbb{Z}} 2^{v \alpha q}\left(\sum_{Q \in \mathcal{D}_{v}}\left|s_{Q}\right|^{p}\right)^{q / p}\right]^{1 / q}
$$


By the same manner, we have

$$
E_{2} \lesssim\left[\sum_{\nu \in \mathbb{Z}} 2^{v \alpha q}\left(\sum_{Q \in \mathcal{D}_{v}}\left|s_{Q}\right|^{p}\right)^{q / p}\right]^{1 / q}
$$

Therefore,

$$
\|f\|_{\dot{B}_{p, q, w}^{\alpha, L}} \lesssim\left[\sum_{v \in \mathbb{Z}} 2^{v \alpha q}\left(\sum_{Q \in \mathcal{D}_{v}}\left|s_{Q}\right|^{p}\right)^{q / p}\right]^{1 / q}
$$

as desired.

4.2. Atomic decompositions for Triebel-Lizorkin spaces $\dot{F}_{p, q, w}^{\alpha, L}$. Our second main result is the following atomic decomposition theorem for the spaces $\dot{F}_{p, q, w}^{\alpha, L}$. More precisely, we prove the following theorem.

THEOREM 4.6. Let $\alpha \in \mathbb{R}, 0<p<\infty, 0<q \leqslant \infty, M \in \mathbb{N}_{+}$and $w \in A_{\infty}$. If $f \in \dot{F}_{p, q, w}^{\alpha, L}$, then there exist a sequence of $(L, M, p, w)$ atoms $\left\{a_{Q}\right\}_{Q \in \mathcal{D}_{v}, v \in \mathbb{Z}}$ and a sequence of coefficients $\left\{s_{Q}\right\}_{Q \in \mathcal{D}_{v}, v \in \mathbb{Z}}$ so that

$$
f=\sum_{v \in \mathbb{Z}} \sum_{Q \in \mathcal{D}_{v}} s_{Q} a_{Q} \quad \text { in } \mathcal{S}_{\infty}^{\prime}
$$

Moreover,

$$
\left\|\left[\sum_{v \in \mathbb{Z}} 2^{v \alpha q}\left(\sum_{Q \in \mathcal{D}_{v}} w(Q)^{-1 / p}\left|s_{Q}\right| \chi_{Q}\right)^{q}\right]^{1 / q}\right\|_{p, w} \lesssim\|f\|_{\dot{F}_{p, q, w}^{\alpha, L}} .
$$

Proof. Recall that in the proof of Theorem 4.2, we have proved the representation

$$
f=\sum_{v \in \mathbb{Z}} \sum_{Q \in \mathcal{D}_{v}} s_{Q} a_{Q} \quad \text { in } \mathcal{S}_{\infty}^{\prime}
$$

where

$$
s_{Q}=w(Q)^{1 / p} \sup _{y \in Q} \int_{2^{-\nu-1}}^{2^{-\nu}}\left|\psi_{M}(t \sqrt{L}) f(y)\right| \frac{d t}{t},
$$

and $a_{Q}=L^{M} b_{Q}$ is an $(L, M, p, w)$ atom defined by

$$
b_{Q}=\frac{1}{s_{Q}} \int_{2^{-v-1}}^{2^{-v}} t^{2 M} \Phi(t \sqrt{L})\left[\psi_{M}(t \sqrt{L}) f \cdot \chi_{Q}\right] \frac{d t}{t} .
$$


It remains to prove (90). Indeed, for any $\lambda>0$, it is easy to see that

$$
w(Q)^{-1 / p} s_{Q} \chi_{Q}=\sup _{y \in Q} \int_{2^{-v-1}}^{2^{-v}}\left|\psi_{M}(t \sqrt{L}) f(y)\right| \frac{d t}{t} \cdot \chi_{Q} \lesssim \chi_{Q} F_{M, \lambda}^{*}(\sqrt{L}) f
$$

where

$$
F_{M, \lambda}^{*}(\sqrt{L}) f(x)=\sup _{y \in X} \frac{\int_{2^{-v-1}}^{2^{-v}}\left|\psi_{M}(t \sqrt{L}) f(y)\right| \frac{d t}{t}}{\left(1+2^{v} d(x, y)\right)^{\lambda}} .
$$

As a consequence,

$$
\sum_{Q \in \mathcal{D}_{v}} w(Q)^{-1 / p}\left|s_{Q}\right| \chi_{Q} \lesssim F_{M, \lambda}^{*}(\sqrt{L}) f .
$$

On the other hand, fixing an $m>\alpha / 2$ and arguing similarly to (55), we show that

$$
\left|F_{M, \lambda}^{*}(\sqrt{L}) f\right| \lesssim \sum_{j \in \mathbb{Z}} 2^{-2 m|\nu-j|} \psi_{j, \lambda}^{*}(\sqrt{L}) f .
$$

Therefore,

$$
\begin{aligned}
& \left\|\left[\sum_{v \in \mathbb{Z}} 2^{v \alpha q}\left(\sum_{Q \in \mathcal{D}_{v}} w(Q)^{-1 / p}\left|s_{Q}\right| \chi_{Q}\right)^{q}\right]^{1 / q}\right\|_{p, w} \\
& \quad \lesssim\left\|\left[\sum_{v \in \mathbb{Z}} 2^{v \alpha q}\left(\sum_{j \in \mathbb{Z}} 2^{-2 m|v-j|} \psi_{j, \lambda}^{*}(\sqrt{L}) f\right)^{q}\right]^{1 / q}\right\|_{p, w} \\
& \quad \lesssim\left\|\left[\sum_{v \in \mathbb{Z}}\left(\sum_{j \in \mathbb{Z}} 2^{-2 m|v-j|+\alpha(v-j)} 2^{j \alpha} \psi_{j, \lambda}^{*}(\sqrt{L}) f\right)^{q}\right]^{1 / q}\right\|_{p, w} .
\end{aligned}
$$

We then apply Young's inequality when $q \geqslant 1$ and the inequality $\left(\sum_{j}\left|a_{j}\right|\right)^{q} \leqslant$ $\sum_{j}\left|a_{j}\right|^{q}$ when $0<q<1$ to find that

$$
\begin{aligned}
& \left\|\left[\sum_{v \in \mathbb{Z}} 2^{v \alpha q}\left(\sum_{Q \in \mathcal{D}_{v}} w(Q)^{-1 / p}\left|s_{Q}\right| \chi_{Q}\right)^{q}\right]^{1 / q}\right\|_{p, w} \\
& \quad \lesssim\left\|\left[\sum_{j \in \mathbb{Z}}\left(2^{j \alpha} \psi_{j, \lambda}^{*}(\sqrt{L}) f\right)^{q}\right]^{1 / q}\right\|_{p, w} \\
& \quad \lesssim\|f\|_{F_{p, q, w}^{\alpha, L}},
\end{aligned}
$$

where, in the last inequality, we use Proposition 3.3.

This completes our proof. 
For the converse direction, we have the following theorem.

THEOREM 4.7. Let $\alpha \in \mathbb{R}, 0<p<\infty, 0<q \leqslant \infty$ and $w \in A_{\infty}$. If

$$
f=\sum_{v \in \mathbb{Z}} \sum_{Q \in \mathcal{D}_{v}} s_{Q} a_{Q} \quad \text { in } \mathcal{S}_{\infty}^{\prime}
$$

where $\left\{a_{Q}\right\}_{Q \in \mathcal{D}_{v}, v \in \mathbb{Z}}$ is a sequence of $(L, M, p, w)$ atoms and $\left\{s_{Q}\right\}_{Q \in \mathcal{D}_{v}, v \in \mathbb{Z}}$ is a sequence of coefficients satisfying

$$
\left\|\left[\sum_{v \in \mathbb{Z}} 2^{v \alpha q}\left(\sum_{Q \in \mathcal{D}_{v}} w(Q)^{-1 / p}\left|s_{Q}\right| \chi_{Q}\right)^{q}\right]^{1 / q}\right\|_{p, w}<\infty,
$$

then $f \in \dot{F}_{p, q, w}^{\alpha, L}$ and

$$
\|f\|_{\dot{F}_{p, q, w}^{\alpha, L}} \lesssim\left\|\left[\sum_{\nu \in \mathbb{Z}} 2^{\nu \alpha q}\left(\sum_{Q \in \mathcal{D}_{v}} w(Q)^{-1 / p}\left|s_{Q}\right| \chi_{Q}\right)^{q}\right]^{1 / q}\right\|_{p, w}
$$

provided $M>\frac{n}{2}+\frac{1}{2} \max \left\{\alpha, \frac{n q_{w}}{1 \wedge p \wedge q}-\alpha\right\}$.

Proof. The proof of this theorem is similar to that of Theorem 4.3. Hence, we just sketch the main ideas. With the same notations as in the proof of Theorem 4.3, from (89), we have

$$
\begin{aligned}
2^{j \alpha}\left|\psi_{j}(\sqrt{L}) f\right| \lesssim & \sum_{\nu: v \geqslant j} 2^{-(\nu-j)(2 M-n \tilde{q} / r-\alpha)} \mathcal{M}_{w, r}\left(\sum_{Q \in \mathcal{D}_{v}} 2^{v \alpha}\left|s_{Q}\right| w(Q)^{-1 / p} \chi_{Q}\right) \\
& +\sum_{\nu: v<j} 2^{-(2 M-\alpha)(j-\nu)} \mathcal{M}_{w, r}\left(\sum_{Q \in \mathcal{D}_{v}} 2^{v \alpha}\left|s_{Q}\right| w(Q)^{-1 / p} \chi_{Q}\right) .
\end{aligned}
$$

By using (10), we conclude that

$$
\begin{aligned}
\|f\|_{\dot{F}_{p, q, w}^{\alpha, L}} & =\left\|\left[\sum_{j \in \mathbb{Z}}\left(2^{j \alpha}\left|\psi_{j}(\sqrt{L}) f\right|\right)^{q}\right]^{1 / q}\right\|_{p, w} \\
& \lesssim\left\|\left[\sum_{v \in \mathbb{Z}} 2^{v \alpha q}\left(\sum_{Q \in \mathcal{D}_{v}} w(Q)^{-1 / p}\left|s_{Q}\right| \chi_{Q}\right)^{q}\right]^{1 / q}\right\|_{p, w} .
\end{aligned}
$$

This completes our proof.

REMARK 4.8. By a careful examination of the proofs of Theorem 4.2 and Theorem 4.6, it is easy to see that each atom $a_{Q}=L^{2 M} b_{Q}$, defined by (85), 
belongs to the spaces of test functions $\mathcal{S}_{\infty}$. As a direct consequence of the atomic decomposition results in these two theorems, the test functions space $\mathcal{S}_{\infty}$ is dense in both $\dot{B}_{p, q, w}^{\alpha, L}$ and $\dot{F}_{p, q, w}^{\alpha, L}$ when $0<p, q<\infty$.

We can mimic Definition 4.1 to define new molecules associated with $L$ as follows: Let $0<p \leqslant \infty, N>0, M \in \mathbb{N}_{+}$and $w \in A_{\infty}$. A function $m$ is said to be an $(L, M, N, p, w)$ molecule if there exists a dyadic cube $Q \in \mathcal{D}$ so that:

(i) $m=L^{M} b$;

(ii) $\left|L^{k} b(x)\right| \leqslant \ell(Q)^{2(M-k)} w(Q)^{-1 / p}\left(1+\frac{\left|x-x_{Q}\right|}{\ell(Q)}\right)^{-N}, k=0, \ldots, 2 M$;

where $B_{Q}$ is a ball associated with $Q$ defined in Remark 2.4.

Then we can adapt the arguments in the proofs of Theorem 4.2 and Theorem 4.6 to obtain the molecular decompositions for our new Besov and Triebel-Lizorkin spaces. However, we do not aim to present the results in this paper and leave the details to the interested reader. We note that the molecular decomposition theorem for the unweighted case was obtained in [37] under the additional assumptions $(\mathrm{H})$ and $(\mathrm{C})$ by using a different approach. See Remark 6.3.

\section{Identifications of our new Besov and Triebel-Lizorkin spaces with known function spaces}

5.1. Coincidence with $L_{w}^{p}(X)$ spaces. We have the following results.

THEOREM 5.1. For $1<p<\infty$ and $w \in A_{p}$, we have

$$
\dot{F}_{p, 2, w}^{0, L}(X) \equiv L_{w}^{p}(X) .
$$

Proof. Arguing similarly to the proof of [3, Theorem 7.2], we prove that for any $m \in \mathbb{N}^{+}$,

$$
\left\|\left[\int_{0}^{\infty}\left|\Psi_{m, t}(L) f\right|^{2} \frac{d t}{t}\right]^{1 / 2}\right\|_{p, w} \sim c_{p, w}\|f\|_{p, w} .
$$

This, along with Corollary 3.8, implies (91).

5.2. Coincidence with the weighted Hardy spaces $\boldsymbol{H}_{\boldsymbol{L}, \boldsymbol{w}}^{\boldsymbol{p}}$. Let $0<p \leqslant 1$ and $w \in A_{\infty}$. The weighted Hardy space $H_{L, w}^{p}$ is defined as the completion of the set

$$
\left\{f \in L^{2}: \mathcal{S}_{L} f \in L_{w}^{p}\right\}
$$


under the norm $\|f\|_{H_{L, w}^{p}}=\left\|\mathcal{S}_{L} f\right\|_{p, w}$ where

$$
\mathcal{S}_{L} f(x)=\left[\int_{0}^{\infty} \int_{d(x, y)<t}\left|t^{2} L e^{-t L^{2}} f(y)\right|^{2} \frac{d \mu(y) d t}{t V(x, t)}\right]^{1 / 2} .
$$

The Hardy spaces $H_{L}^{1}$ was initiated in [2]. See also [32]. The theory of Hardy spaces associated with operators satisfying Davies-Gaffney estimates $H_{L}^{1}$ was established in [44]. The weighted version for $H_{L, w}^{p}$ was investigated in [14]. From Proposition 3.14, we obtain the following.

THEOREM 5.2. Let $0<p \leqslant 1$ and $w \in A_{\infty}$. Then we have

$$
H_{L, w}^{p} \equiv \dot{F}_{p, 2, w}^{0, L} \text {. }
$$

Moreover, we have an interesting characterization for the weighted Hardy spaces.

Proposition 5.3. Let $0<p \leqslant 1$ and $w \in A_{\infty}$ and let $\psi$ be a partition of unity and $\varphi \in \mathscr{S}_{1}(\mathbb{R})$. Then for any $f \in L^{2} \cap H_{L, w}^{p}$, we have

$$
\begin{aligned}
\|f\|_{H_{L, w}^{p}} & \sim\left\|\left[\int_{0}^{\infty}|\psi(t \sqrt{L}) f|^{2} \frac{d t}{t}\right]^{1 / 2}\right\|_{p, w} \sim\left\|\left[\int_{0}^{\infty}|\varphi(t \sqrt{L}) f|^{2} \frac{d t}{t}\right]^{1 / 2}\right\|_{p, w} \\
& \sim\left\|\mathcal{G}_{\lambda, 2}(\psi(t \sqrt{L}) f)\right\|_{p, w} \sim\left\|\mathcal{S}_{2}(\psi(t \sqrt{L}) f)\right\|_{p, w} \\
& \sim\left\|\mathcal{G}_{\lambda, 2}(\varphi(t \sqrt{L}) f)\right\|_{p, w} \sim\left\|\mathcal{S}_{2}(\varphi(t \sqrt{L}) f)\right\|_{p, w},
\end{aligned}
$$

where $\mathcal{S}_{2}$ and $\mathcal{G}_{\lambda, 2}$ are square functions defined in (64) and (65).

Proof. The proposition follows immediately from Theorems 3.5 and 3.6 and Propositions 3.13 and 3.14.

REMARK 5.4. These equivalent norm estimates for weighted Hardy spaces $H_{L, w}^{p}$ are new. It is worth noticing that in the particular case when $\varphi(\xi)=\xi^{2 M} e^{-\xi^{2}}$ with $M \geqslant 1$, the equivalent estimate in Proposition 5.3

$$
\|f\|_{H_{L, w}^{p}} \sim\left\|\left[\int_{0}^{\infty}|\varphi(t \sqrt{L}) f|^{2} \frac{d t}{t}\right]^{1 / 2}\right\|_{p, w}
$$

reads

$$
\|f\|_{H_{L, w}^{p}} \sim\left\|\left[\int_{0}^{\infty}\left|\left(t^{2} L\right)^{M} e^{-t^{2} L} f\right|^{2} \frac{d t}{t}\right]^{1 / 2}\right\|_{p, w} .
$$

Note that this estimate was proved in [41] (see also [33]) for $M=1$. 


\subsection{Coincidence with the weighted BMO spaces $\mathrm{BMO}_{L, w}(X)$}

Definition 5.5. Let $w \in A_{\infty}$. The function $f \in \mathcal{S}^{\prime} \cap L_{\text {loc }}^{1}(X)$ is said to be in $\mathrm{BMO}_{L, w}(X)$, the weighted space of functions of bounded mean oscillation associated with $L$, if

$$
\|f\|_{\mathrm{BMO}_{L, w}(X)}:=\sup _{B: \text { balls }} \frac{1}{w(B)} \int_{B}\left|\left(I-e^{-r_{B}^{2} L}\right) f(x)\right| d \mu(x)<\infty .
$$

The unweighted BMO space $\mathrm{BMO}_{L}(X)$ associated with operators $L$ was first introduced by [31]. The weighted version was studied in $[19,40]$.

It was proved [40, Theorem 5.5] that when $L=-\Delta$ on $\mathbb{R}^{n}$, we have

$$
\mathrm{BMO}_{-\Delta, w}\left(\mathbb{R}^{n}\right)=\mathrm{BMO}_{w}\left(\mathbb{R}^{n}\right)
$$

for all $w \in A_{1} \cap R H_{2}$, where

$$
\mathrm{BMO}_{w}\left(\mathbb{R}^{n}\right)=\left\{f \in L_{\text {loc }}^{1}:\|f\|_{\mathrm{BMO}_{w}}:=\sup _{B: \text { balls }} \frac{1}{w(B)} \int_{B}\left|f-f_{B}\right| d x<\infty\right\} .
$$

We now prove the coincidence between the weighted $\mathrm{BMO}$ space $\mathrm{BMO}_{L, w}(X)$ and the weighted Triebel-Lizorkin space $\dot{F}_{\infty, 2, w}^{0, L}(X)$.

THEOREM 5.6. We have the following identities:

(a) $\mathrm{BMO}_{L}(X) \equiv \dot{F}_{\infty, 2}^{0, L}(X)$ in the sense that if $f \in \mathrm{BMO}_{L}(X)$, then $f \in \dot{F}_{\infty, 2}^{0, L}(X)$; conversely, if $f \in \dot{F}_{\infty, 2}^{0, L}(X)$, then there exists $\rho \in \mathscr{P}$ so that $f-\rho \in$ $\mathrm{BMO}_{L}(X)$.

(b) Let $w \in A_{1} \cap R H_{2}$. Then we have $\mathrm{BMO}_{w}\left(\mathbb{R}^{n}\right) \equiv \dot{F}_{\infty, 2, w}^{0,-\Delta}\left(\mathbb{R}^{n}\right)$ in the similar sense to that of (a).

Proof. (a) It was proved [47, Theorem 4.2] that

$$
\sup _{x \in X, t>0}\left(\frac{1}{V(x, t)} \int_{B(x, t)} \int_{0}^{t}\left|s^{2} L e^{-s^{2} L} e^{-s^{2} L} f\right|^{2} \frac{d s}{s} d \mu(y)\right)^{1 / 2} \sim\|f\|_{\mathrm{BMO}_{L, w}(X)} .
$$

This, along with Theorem 3.21, implies the assertion.

(b) It was proved in [40] that

$\|f\|_{\mathrm{BMO}_{w}\left(\mathbb{R}^{n}\right)} \sim \sup _{x \in X, t>0}\left(\frac{|B(x, t)|}{w(B(x, t))^{2}} \int_{B(x, t)} \int_{0}^{t}\left|s^{2} \Delta e^{s^{2} \Delta}\left(I-e^{s^{2} \Delta}\right) f\right|^{2} \frac{d s}{s} d y\right)^{1 / 2}$.

Using this and Theorem 3.21, we derive part (b). 
5.4. Coincidence with the weighted Sobolev spaces $\dot{\boldsymbol{W}}_{p, w}^{s, L}$. For $s \in \mathbb{R}$, we define $L^{s / 2}: \mathcal{S}_{\infty} \rightarrow \mathcal{S}_{\infty}$ by setting

$$
L^{s / 2} f=\frac{1}{\Gamma(m-s / 2)} \int_{0}^{\infty} t^{-s / 2}(t L)^{m} e^{-t L} f \frac{d t}{t}
$$

for any $m \in \mathbb{N}, m>s / 2$.

Arguing similarly to Proposition 2.10, we can prove that the right-hand side in (95) converges in $\mathcal{S}_{\infty}$. Moreover, by integration by part, we can see that

$$
\frac{1}{\Gamma(m-s / 2)} \int_{0}^{\infty} t^{-s / 2}(t L)^{m} e^{-t L} f \frac{d t}{t}=\frac{1}{\Gamma(\ell-s / 2)} \int_{0}^{\infty} t^{-s / 2}(t L)^{\ell} e^{-t L} f \frac{d t}{t}
$$

for any $m, \ell \in \mathbb{N}, m, \ell>s / 2$.

Therefore, $L^{s / 2}$ given by (95) is well defined as an operator from $\mathcal{S}_{\infty}$ into $\mathcal{S}_{\infty}$. Moreover, it is easy to check that

$$
L^{\alpha}\left[L^{\beta} f\right]=L^{\alpha+\beta} f, \quad \forall f \in \mathcal{S}_{\infty} .
$$

We now define the weighted Sobolev spaces $\dot{W}_{p, w}^{s, L}$ as follows: Let $s \in \mathbb{R}$, $1<p<\infty$ and $w \in A_{\infty}$. The weighted Sobolev space $\dot{W}_{p, w}^{s, L}$ is defined as the completion of the set

$$
\left\{f \in \mathcal{S}_{\infty}:\left\|L^{s / 2} f\right\|_{p, w}<\infty\right\}
$$

under the norm $\|f\|_{\dot{W}_{p, w}^{s, L}}=\left\|L^{s / 2} f\right\|_{p, w}$.

Theorem 5.7. Let $s \in \mathbb{R}, 1<p<\infty$ and $w \in A_{\infty}$. Then we have

$$
\dot{W}_{p, w}^{s, L} \equiv \dot{F}_{p, 2, w}^{s, L} .
$$

Proof. From Remark 4.8, we need only to show that

$$
\dot{W}_{p, w}^{s, L} \cap \mathcal{S}_{\infty} \equiv \dot{F}_{p, 2, w}^{s, L} \cap \mathcal{S}_{\infty} .
$$

Indeed, let $f \in \dot{F}_{p, 2, w}^{s, L} \cap \mathcal{S}_{\infty}$ and $\psi$ be a partition of unity. Then by Theorem 5.1 and the spectral theory, for $g \in L_{w^{1-p^{\prime}}}^{p^{\prime}}$,

$$
\begin{aligned}
\left|\left\langle L^{s / 2} f, g\right\rangle\right| & =\left|c_{\psi} \int_{X} \int_{0}^{\infty} \psi^{3}(t \sqrt{L}) L^{s / 2} f(x) g(x) \frac{d t}{t} d \mu(x)\right| \\
& =\left|c_{\psi} \int_{X} \int_{0}^{\infty} \psi^{2}(t \sqrt{L}) L^{s / 2} f(x) \psi(t \sqrt{L}) g(x) \frac{d t}{t} d \mu(x)\right|
\end{aligned}
$$




$$
\begin{gathered}
\lesssim c_{\psi}\left\|\left[\int_{0}^{\infty}\left|\psi(t \sqrt{L})^{2} L^{s / 2} f\right|^{2} \frac{d t}{t}\right]^{1 / 2}\right\|_{p, w} \\
\times\left\|\left[\int_{0}^{\infty}|\psi(t \sqrt{L}) g|^{2} \frac{d t}{t}\right]^{1 / 2}\right\| \|_{p^{\prime}, w^{1-p^{\prime}}},
\end{gathered}
$$

where $c_{\psi}=\left[\int_{0}^{\infty} \psi^{3}(s) \frac{d s}{s}\right]^{-1}$, and we use Hölder's inequality in the last inequality.

On the other hand, since $w \in A_{p}, w^{1-p^{\prime}} \in A_{p^{\prime}}$. By Theorems 5.1 and 3.5, we have

$$
\left\|\left[\int_{0}^{\infty}|\psi(t \sqrt{L}) g|^{2} \frac{d t}{t}\right]^{1 / 2}\right\|_{p^{\prime}, w^{1-p^{\prime}}} \sim\|g\|_{p^{\prime}, w^{1-p^{\prime}}} .
$$

Therefore,

$$
\left|\left\langle L^{s / 2} f, g\right\rangle\right| \lesssim\|g\|_{p^{\prime}, w^{1-p^{\prime}}}\left\|\left[\int_{0}^{\infty}\left|\psi(t \sqrt{L}) L^{s / 2} f\right|^{2} \frac{d t}{t}\right]^{1 / 2}\right\|_{p, w} .
$$

Using (95), we have

$$
\begin{aligned}
\psi^{2}(t \sqrt{L}) L^{s / 2} f(x) & =\frac{1}{\Gamma(m-s / 2)} \int_{0}^{\infty} u^{-s / 2} \psi(t \sqrt{L})(u L)^{m} e^{-u L}(\psi(t \sqrt{L}) f) \frac{d u}{u} \\
& =\int_{0}^{t^{2}} \cdots+\int_{t^{2}}^{\infty} \cdots=: E_{1}(x, t)+E_{2}(x, t) .
\end{aligned}
$$

Fix $\lambda>\max \left\{n / q, n q_{w} / p\right\}$ and $M>(\lambda+s) / 2$. By Lemma 2.6, we have, for $N>n$

$$
\begin{aligned}
\left|E_{1}(x, t)\right| \lesssim & \int_{0}^{t^{2}} \int_{X} u^{-s / 2}\left(\frac{t^{2}}{u}\right)^{M} \frac{1}{V(x, u)}\left(1+\frac{d(x, y)}{u}\right)^{-N-\lambda} \\
& \times|\psi(t \sqrt{L}) f(y)| d \mu(y) \frac{d t}{t} \\
\lesssim & \int_{0}^{t^{2}} \int_{X} u^{-s / 2}\left(\frac{t^{2}}{u}\right)^{M-\lambda / 2} \frac{1}{V(x, u)}\left(1+\frac{d(x, y)}{u}\right)^{-N} \\
& \times \psi_{\lambda}^{*}(t \sqrt{L}) f(x) d \mu(y) \frac{d t}{t} \\
\lesssim & t^{-s} \psi_{\lambda}^{*}(t \sqrt{L}) f(x) .
\end{aligned}
$$

Similarly, we have

$$
\left|E_{2}(x, t)\right| \lesssim t^{-s} \psi_{\lambda}^{*}(t \sqrt{L}) f(x)
$$

Hence,

$$
\left|\psi^{2}(t \sqrt{L}) L^{s / 2} f(x)\right| \lesssim t^{-s} \psi_{\lambda}^{*}(t \sqrt{L}) f(x)
$$


Inserting this into (97), then using Theorem 3.5, we get that

$$
\begin{aligned}
\left|\left\langle L^{s / 2} f, g\right\rangle\right| & \lesssim\|g\|_{p^{\prime}, w^{1-p^{\prime}}}\left\|\left[\int_{0}^{\infty}\left(t^{-s} \psi_{\lambda}^{*}(t \sqrt{L}) f\right)^{2} \frac{d t}{t}\right]^{1 / 2}\right\|_{p, w} \\
& \lesssim\|g\|_{p^{\prime}, w^{1-p^{\prime}}}\|f\|_{\dot{F}_{p, 2, w}^{0, L}} .
\end{aligned}
$$

This implies $\left\|L^{s / 2} f\right\|_{p, w} \lesssim\|f\|_{\dot{F}_{p, 2, w}^{0, L}}$. Hence, $\dot{F}_{p, 2, w}^{s, L} \cap \mathcal{S}_{\infty} \hookrightarrow \dot{W}_{p, w}^{s, L} \cap \mathcal{S}_{\infty}$.

Conversely, let $\psi \in \mathscr{S}(\mathbb{R})$ so that $\psi^{3}$ is a partition of unity. Then for $f \in \dot{W}_{p, w}^{s, L} \cap$ $\mathcal{S}_{\infty}$, by (96), we have

$$
\begin{aligned}
\|f\|_{\dot{F}_{p, 2, w}^{s, L}} & :=\left\|\left[\sum_{j \in \mathbb{Z}}\left(2^{-j s}\left|\psi^{3}\left(2^{-j} \sqrt{L}\right) f\right|\right)^{2}\right]^{1 / 2}\right\|_{p, w} \\
& \lesssim\left\|\left[\sum_{j \in \mathbb{Z}}\left(2^{-j s}\left|L^{-s / 2} \psi^{3}\left(2^{-j} \sqrt{L}\right)\left(L^{s / 2} f\right)\right|\right)^{2}\right]^{1 / 2}\right\|_{p, w} .
\end{aligned}
$$

Arguing similarly to the proof of (98), we have, for each $j \in \mathbb{Z}$,

$$
\left|L^{-s / 2} \psi^{3}\left(2^{-j} \sqrt{L}\right)\left(L^{s / 2} f\right)\right| \lesssim 2^{j s} \psi_{j, \lambda}^{*}(\sqrt{L})\left(L^{s / 2} f\right) .
$$

Therefore,

$$
\left.\|f\|_{\dot{F}_{p, 2, w}^{s, L}} \lesssim \|\left[\sum_{j \in \mathbb{Z}}\left|\psi_{j, \lambda}^{*}(\sqrt{L})\left(L^{s / 2} f\right)\right|\right)^{2}\right]^{1 / 2} \|_{p, w} .
$$

This, together with Propositions 2.16 and 3.3, yields

$$
\|f\|_{\dot{F}_{p, 2, w}^{s, L}} \lesssim\left\|L^{s / 2} f\right\|_{\dot{F}_{p, 2, w}^{0, L}} .
$$

On the other hand, from Theorem 5.1, we have $\left\|L^{s / 2} f\right\|_{\dot{F}_{p, 2, w}^{0, L}} \sim\left\|L^{s / 2} f\right\|_{p, w}$. Hence,

$$
\|f\|_{\dot{F}_{p, L, w}^{s, L}} \lesssim\left\|L^{s / 2} f\right\|_{p, w} .
$$

This completes our proof.

5.5. Coincidence with weighted Hardy-Sobolev spaces $\boldsymbol{H} \dot{\boldsymbol{S}}_{p, w}^{s, L}$. For $s \in \mathbb{R}$, $0<p \leqslant 1$ and $w \in A_{\infty}$ the weighted Hardy-Sobolev space $H \dot{S}_{p, w}^{s, L}$ is defined as the completion of the set

$$
\left\{f \in \mathcal{S}_{\infty}:\left\|L^{s / 2} f\right\|_{H_{L, w}^{p}}<\infty\right\}
$$

under the norm $\|f\|_{H \dot{S}_{p, w}^{s, L}}=\left\|L^{s / 2} f\right\|_{H_{L, w}^{p}}$, where $H_{L, w}^{p}$ is the weighted Hardy space defined as in Section 5.2. 
THEOREM 5.8. Let $s \in \mathbb{R}, 0<p \leqslant 1$ and $w \in A_{\infty}$. Then we have

$$
H \dot{S}_{p, w}^{s, L} \equiv \dot{F}_{p, 2, w}^{s, L}
$$

Proof. Since $\mathcal{S}_{\infty}$ is dense in both spaces, we need only to verify that

$$
H \dot{S}_{p, w}^{s, L} \cap \mathcal{S}_{\infty} \equiv \dot{F}_{p, 2, w}^{s, L} \cap \mathcal{S}_{\infty}
$$

We first verify that $\dot{F}_{p, 2, w}^{s, L} \cap \mathcal{S}_{\infty} \subset H \dot{S}_{p, w}^{s, L} \cap \mathcal{S}_{\infty}$. Indeed, if $f \in \dot{F}_{p, 2, w}^{s, L} \cap \mathcal{S}_{\infty}$, then by Theorems 5.2 and 3.5, we have

$$
\|f\|_{H \dot{S}_{p, w}^{s, L}}=\left\|L^{s / 2} f\right\|_{H_{L, w}^{p}} \sim\left\|\left[\int_{0}^{\infty}\left|\psi(t \sqrt{L})\left(L^{s / 2} f\right)\right|\right]^{1 / 2}\right\|_{p, w},
$$

where $\psi \in \mathscr{S}(\mathbb{R})$ so that $\psi^{3}$ is a partition of unity.

At this stage, we argue similarly to the proof of Theorem 5.8 to obtain that

$$
\|f\|_{H \dot{S}_{p, w}^{s, L}} \lesssim\|f\|_{\dot{F}_{p, 2, w}^{s, L}}
$$

which implies $\dot{F}_{p, 2, w}^{s, L} \cap \mathcal{S}_{\infty} \subset H \dot{S}_{p, w}^{s, L} \cap \mathcal{S}_{\infty}$.

The converse direction is similar and we omit the details.

\section{Comparison with classical Besov and Triebel-Lizorkin spaces}

Definition 6.1. Let $0<p \leqslant \infty, w \in A_{\infty}$ and $\epsilon>0$. A function $a$ is said to be a $(p, w, \epsilon)$ atom if there exists a dyadic cube $Q \in \mathcal{D}_{v}$ such that:

(i) $\operatorname{supp} a \subset 3 B_{Q}$;

(ii) $|a(x)| \leqslant w(Q)^{-1 / p}$;

(iii) $|a(x)-a(y)| \leqslant w(Q)^{1 / p}\left(\frac{d(x, y)}{2^{-v}}\right)^{\epsilon}$;

(iv) $\int a(x) d \mu(x)=0$.

Let $0<p<\infty, w \in A_{\infty}$ and $\epsilon>0$. We say that a function $f$ has a $(p, w, \epsilon)$ atomic representation of Besov type if

$$
f=\sum_{\nu \in \mathbb{Z}} \sum_{Q \in \mathcal{D}_{v}} s_{Q} a_{Q} \quad \text { in } L^{2}
$$


where $\left\{a_{Q}\right\}$ is a sequence of $(p, w, \epsilon)$ atoms and $s:=\left\{s_{Q}\right\}$ is a sequence of numbers satisfying

$$
\left[\sum_{v \in Z} 2^{v \alpha q}\left(\sum_{Q \in \mathcal{D}_{v}}\left|s_{Q}\right|^{p}\right)^{q / p}\right]^{1 / q}<\infty
$$

Then the weighted Besov space is defined as follows.

Definition 6.2. Let $\alpha \in(-1,1), 0<p, q<\infty$ and $w \in A_{\infty}$. The weighted Besov space $B_{p, q, w}^{\alpha}$ is defined as the completion of the set of all $L^{2}$-functions having a $(p, w, \epsilon)$ atomic representation of Besov type under the norm

$$
\|f\|_{\dot{B}_{p, q, w}^{\alpha}}=\inf \left\{\left[\sum_{\nu \in \mathbb{Z}} 2^{v \alpha q}\left(\sum_{Q \in \mathcal{D}_{v}}\left|s_{Q}\right|^{p}\right)^{q / p}\right]^{1 / q}: f=\sum_{v \in \mathbb{Z}} \sum_{Q \in \mathcal{D}_{v}} s_{Q} a_{Q}\right\} .
$$

Similarly, the weighted Triebel-Lizorkin spaces $F_{p, q, w}^{\alpha}$ is defined by replacing the quantity

$$
\left[\sum_{v \in \mathbb{Z}} 2^{v \alpha q}\left(\sum_{Q \in \mathcal{D}_{v}}\left|s_{Q}\right|^{p}\right)^{q / p}\right]^{1 / q},
$$

by

$$
\left\|\left[\sum_{v \in \mathbb{Z}} 2^{v \alpha q}\left(\sum_{Q \in \mathcal{D}_{v}} w(Q)^{-1 / p}\left|s_{Q}\right| \chi_{Q}\right)^{q}\right]^{1 / q}\right\|_{p, w} .
$$

REMARK 6.3. Up to now, we have stated and proved our results under rather mild assumptions on $L$; namely, we have assumed that $L$ is a nonnegative self-adjoint operator for which its heat kernel $p_{t}(x, y)$ satisfies the Gaussian upper bound (GE). In some applications below, we will require $L$ also to satisfy one or both of the following additional conditions:

(H) There exists $\delta_{0} \in(0,1]$ so that

$$
\left|p_{t}(x, y)-p_{t}(\bar{x}, y)\right| \lesssim\left(\frac{d(x, \bar{x})}{\sqrt{t}}\right)^{\delta_{0}} \frac{1}{V(x, \sqrt{t})} \exp \left(-\frac{d(x, y)^{2}}{c t}\right)
$$

whenever $d(x, \bar{x})<\sqrt{t}$. (Hölder Continuity Property.)

(C) $\int_{X} p_{t}(x, y) d \mu(x)=1$ for all $y \in X$ and $t>0$. (Conservation Property.)

We have the following estimate. 
LEMMA 6.4. Let $\Phi$ be a function as in Lemma 2.1. Also assume that L satisfies (H). Then for any $M \in \mathbb{N}$, we have

$$
\left|K_{\left(t^{2} L\right)^{M} \Phi(t \sqrt{L})}(x, y)-K_{\left(t^{2} L\right)^{M} \Phi(t \sqrt{L})}(\bar{x}, y)\right| \lesssim\left(\frac{d(x, \bar{x})}{t}\right)^{\delta_{0}} \frac{1}{V(x, t)}
$$

whenever $d(x, \bar{x})<t$.

Proof. The proof of this lemma is similar to that of [57, Theorem 1]. We leave the details to the interested reader.

The following theorem is the main result of this section.

THEOREM 6.5. Assume that $L$ also satisfies $(H)$ and $(C)$. Then we have

$$
\dot{B}_{p, q, w}^{\alpha, L} \equiv \dot{B}_{p, q, w}^{\alpha}
$$

for all $\alpha \in\left(-\delta_{0}, \delta_{0}\right), 0<p, q<\infty$ and $w \in A_{\infty}$ such that $\alpha+n+\delta_{0}>\frac{n q_{w}}{p}$; and

$$
\dot{F}_{p, q, w}^{\alpha, L} \equiv \dot{F}_{p, q, w}^{\alpha}
$$

for all $\alpha \in\left(-\delta_{0}, \delta_{0}\right), 0<p, q<\infty$ and $w \in A_{\infty}$ such that $\alpha+n+\delta_{0}>\frac{n q_{w}}{p}$.

Proof. We will only prove $\dot{F}_{p, q, w}^{\alpha, L} \equiv \dot{F}_{p, q, w}^{\alpha}$ since the proof of $\dot{B}_{p, q, w}^{\alpha, L} \equiv \dot{B}_{p, q, w}^{\alpha}$ can be done similarly.

We split the proof into two steps.

Step 1: Proof of $\dot{F}_{p, q, w}^{\alpha, L} \cap L^{2} \hookrightarrow \dot{F}_{p, q, w}^{\alpha} \cap L^{2}$. To do this, we will employ the same notations as in the proof of Theorem 5.6. If $f \in \dot{F}_{p, q, w}^{\alpha, L} \cap L^{2}$, then repeating the proof of Theorem 5.6, we can find a sequence of $(L, M, p, w)$ atoms $\left\{a_{Q}\right\}_{Q \in \mathcal{D}_{v}, v \in \mathbb{Z}}$ and a sequence of coefficients $\left\{s_{Q}\right\}_{Q \in \mathcal{D}_{v}, v \in \mathbb{Z}}$ so that

$$
f=\sum_{v \in \mathbb{Z}} \sum_{Q \in \mathcal{D}_{v}} s_{Q} a_{Q} \quad \text { in } L^{2}
$$

where

$$
a_{Q}=\frac{1}{s_{Q}} \int_{2^{-v-1}}^{2^{-v}}\left(t^{2} L\right)^{M} \Phi(t \sqrt{L})\left[\psi_{M}(t \sqrt{L}) f \cdot \chi_{Q}\right] \frac{d t}{t},
$$

and, moreover,

$$
\left\|\left[\sum_{\nu \in \mathbb{Z}} 2^{v \alpha q}\left(\sum_{Q \in \mathcal{D}_{v}} w(Q)^{-1 / p}\left|s_{Q}\right| \chi_{Q}\right)^{q}\right]^{1 / q}\right\|_{p, w} \lesssim\|f\|_{\dot{F}_{p, q, w}^{\alpha, L}} .
$$


We now claim that each $(L, M, p, w)$ atom is also $\left(p, w, \delta_{0}\right)$ atom. Indeed, it is clear that each $(L, M, p, w)$ atom satisfies (i) and (ii) in Definition 6.1. The argument as in Lemma 9.1 in [23] implies that an $(L, M, p, w)$ atom satisfies (iv) in Definition 6.1. The condition (iii) in Definition 6.1 can be verified by making use of Lemma 6.4, and, hence, we omit the details. This completes the first step.

Step 2: Proof of $\dot{F}_{p, q, w}^{\alpha} \cap L^{2} \hookrightarrow \dot{F}_{p, q, w}^{\alpha, L} \cap L^{2}$. To do this, we need the following estimates.

LEMMA 6.6. Let $a_{Q}$ be a $\left(p, w, \delta_{0}\right)$ atom associated with some dyadic cube $Q \in$ $\mathcal{D}_{v}, v \in \mathbb{Z}$. Then, for any $N>0$, we have the following:

(i) $\left|t^{2} L e^{-t^{2} L} a_{Q}(x)\right| \lesssim w(Q)^{-1 / p}\left(\frac{t}{2^{-\nu}}\right)^{\delta_{0}}\left(1+\frac{d\left(x, x_{Q}\right)}{2^{-\nu}}\right)^{-N}$, for all $t \leqslant 2^{-\nu}$;

(ii) $\left|t^{2} L e^{-t^{2} L} a_{Q}(x)\right| \lesssim\left(\frac{2^{-v}}{t}\right)^{\delta_{0}} \frac{V(Q)}{V\left(x_{Q}, t\right)}\left(1+\frac{d\left(x, x_{Q}\right)}{2^{-v}}\right)^{-N}$, for all $2^{-v} \leqslant t$.

Proof. We just sketch the main ideas. Denote by $q_{t}(x ; y)$ the kernel of $t L e^{-t L}$. (i) If $x \in 6 B_{Q}$, then from Lemma 2.6, we have

$$
\begin{aligned}
& \left|t^{2} L e^{-t^{2} L} a_{Q}(x)\right| \\
& \quad \lesssim w(Q)^{-1 / p} \int_{X} \frac{1}{V(y, t)}\left(1+\frac{d(x, y)}{t}\right)^{-K-\delta_{0}}\left(\frac{d(x, y)}{2^{-v}}\right)^{\delta_{0}} d \mu(y) \\
& \quad \lesssim w(Q)^{-1 / p}\left(\frac{t}{2^{-v}}\right)^{\delta_{0}} \\
& \quad \sim w(Q)^{-1 / p}\left(\frac{t}{2^{-v}}\right)^{\delta_{0}}\left(1+\frac{d\left(x, x_{Q}\right)}{2^{-v}}\right)^{-N}\left(\text { since } y \in 6 B_{Q}\right) .
\end{aligned}
$$

If $x \notin 6 B_{Q}$, then we have $d(x, y) \sim d\left(x, x_{Q}\right) \geqslant 2^{-v}$. This, together with Lemma 2.2, implies that

$$
\begin{aligned}
\left|t^{2} L e^{-t^{2} L} a_{Q}(x)\right| \lesssim & w(Q)^{-1 / p} \int_{3 B_{Q}} \frac{1}{V(y, t)}\left(1+\frac{d(x, y)}{t}\right)^{-K-N-\delta_{0}}\left|a_{Q}(y)\right| d \mu(y) \\
\lesssim & w(Q)^{-1 / p}\left(\frac{t}{2^{-v}}\right)^{\delta_{0}}\left(1+\frac{d(x, y)}{2^{-v}}\right)^{-N} \\
& \times \int_{3 B_{Q}} \frac{1}{V(y, t)}\left(1+\frac{d(x, y)}{t}\right)^{-K} d \mu(y) \\
\lesssim & w(Q)^{-1 / p}\left(\frac{t}{2^{-v}}\right)^{\delta_{0}}\left(1+\frac{d(x, y)}{2^{-v}}\right)^{-N}
\end{aligned}
$$


where, in the last inequality, we use Lemma 2.2.

This completes the proof of (i).

(ii) By (iv) in Definition 6.1, we can write

$$
\left|t^{2} L e^{-t^{2} L} a_{Q}(x)\right|=\left|\int_{3 B_{Q}}\left[q_{t^{2}}(x, y)-q_{t^{2}}\left(x, x_{Q}\right)\right] a_{Q}(y) d \mu(y)\right| .
$$

Since $q_{t}(x, y)=2 \int_{X} q_{t / 2}(x, z) p_{t / 2}(z, y) s d \mu(z)$, we see that $q_{t}(x, y)$ satisfies (99). Hence,

$$
\begin{aligned}
& \left|t^{2} L e^{-t^{2} L} a_{Q}(x)\right| \\
& \quad \lesssim \int_{3 B_{Q}}\left(\frac{d\left(y, x_{Q}\right)}{t}\right)^{\delta_{0}} \frac{1}{V\left(x_{Q}, t\right)}\left(1+\frac{d\left(x, x_{Q}\right)}{t}\right)^{-N}\left|a_{Q}(y)\right| d \mu(y) \\
& \quad \lesssim w(Q)^{-1 / p}\left(\frac{2^{-v}}{t}\right)^{\delta_{0}} \frac{V(Q)}{V\left(x_{Q}, t\right)}\left(1+\frac{d\left(x, x_{Q}\right)}{t}\right)^{-N} .
\end{aligned}
$$

This completes the proof of (ii).

We now turn to the proof of Step 2. Using Lemma 6.6 and arguing similarly to (89), we have

$$
\begin{aligned}
\left|t^{2} L e^{-t^{2} L} f\right| \lesssim & \sum_{v: v \geqslant j} 2^{-(\nu-j)\left(\delta_{0}+n-n \tilde{q} / r\right)} \mathcal{M}_{w, r}\left(\sum_{Q \in \mathcal{D}_{v}}\left|s_{Q}\right| w(Q)^{-1 / p} \chi_{Q}\right) \\
& +\sum_{v: v<j} 2^{-\delta_{0}(j-v)} \mathcal{M}_{w, r}\left(\sum_{Q \in \mathcal{D}_{v}}\left|s_{Q}\right| w(Q)^{-1 / p} \chi_{Q}\right) .
\end{aligned}
$$

At this stage, the argument in the proof of Theorem 4.7 (see also Theorem 4.3) shows that $f \in \dot{F}_{p, q, w}^{\alpha, L}$ provided that $\alpha+n+\delta_{0}>\frac{n q_{w}}{p \wedge q}$.

REMARK 6.7. Some comments for the condition $\alpha+n+\delta_{0}>\frac{n q_{w}}{p \wedge q}$ are in order:

(i) If $1 \leqslant p, q<\infty$, the identities (100) and (101) hold true for all $\alpha \in\left(-\delta_{0}, \delta_{0}\right)$ and $w \in A_{\infty}$ with $q_{w}<\min \{p, q\} \times \frac{n+\delta_{0}+\alpha}{n}$.

(ii) If $w \equiv 1$, the identities (100) and (101) hold true for all $\frac{n}{n+\delta_{0}}<p, q<\infty$ and $\frac{n}{p \wedge q}-n-\delta_{0}<\alpha<\delta_{0}$.

\section{Applications}

The theory of Besov and Triebel-Lizorkin spaces have a wide range of applications. See, for example, $[7,20,28]$ and the references therein. In this 
section, we just give two applications to the fractional power and the spectral multipliers. Further application would be an upcoming project and will be investigated in the future.

\subsection{Fractional powers}

THEOREM 7.1. Let $s \in \mathbb{R}$ and let $L^{s / 2}$ be defined as in (95). Then for $\alpha \in \mathbb{R}$ and $w \in A_{\infty}$, the fractional integral $L^{s / 2}$ maps continuously from $\dot{B}_{p, q, w}^{\alpha, L}$ into $\dot{B}_{p, q, w}^{\alpha+s, L}$ for $0<p, q \leqslant \infty$ and from $\dot{F}_{p, q, w}^{\alpha, L}$ into $\dot{F}_{p, q, w}^{\alpha+s, L}$ for $0<p<\infty$ and $0<q \leqslant \infty$.

Proof. Let $\psi$ be a partition of unity and let $\varphi \in \mathscr{S}(\mathbb{R})$ be supported in $[1 / 4,4]$ such that $\varphi=1$ on $[1 / 2,2]$. For $f \in \dot{F}_{p, q, w}^{\alpha, L}(X)$, using (95), we have

$$
\begin{aligned}
\psi(t \sqrt{L}) L^{s / 2} f(x) & =\frac{1}{\Gamma(m-s / 2)} \int_{0}^{\infty} u^{-s / 2} \varphi(t \sqrt{L})(u L)^{m} e^{-u L}(\psi(t \sqrt{L}) f) \frac{d u}{u} \\
& =\int_{0}^{t^{2}} \cdots+\int_{t^{2}}^{\infty} \ldots=: I_{1}(x, t)+I_{2}(x, t) .
\end{aligned}
$$

Fix $\lambda>\max \left\{n / q, n q_{w} / p\right\}$ and $M>(\lambda+s) / 2$. By Lemma 2.6, we have, for $N>n$,

$$
\begin{aligned}
\left|I_{1}(x, t)\right| \lesssim & \int_{0}^{t^{2}} \int_{X} u^{-s / 2}\left(\frac{t^{2}}{u}\right)^{M} \frac{1}{V(x, u)}\left(1+\frac{d(x, y)}{u}\right)^{-N-\lambda} \\
& \times|\psi(t \sqrt{L}) f(y)| d \mu(y) \frac{d t}{t} \\
\lesssim & \int_{0}^{t^{2}} \int_{X} u^{-s / 2}\left(\frac{t^{2}}{u}\right)^{M-\lambda / 2} \frac{1}{V(x, u)}\left(1+\frac{d(x, y)}{u}\right)^{-N} \\
& \times \psi_{\lambda}^{*}(t \sqrt{L}) f(x) d \mu(y) \frac{d t}{t} \\
\lesssim & t^{-s} \psi_{\lambda}^{*}(t \sqrt{L}) f(x) .
\end{aligned}
$$

Similarly,

$$
\left|I_{2}(x, t)\right| \lesssim t^{-s} \psi_{\lambda}^{*}(t \sqrt{L}) f(x)
$$

Hence,

$$
\left|\psi(t \sqrt{L}) L^{s / 2} f(x)\right| \lesssim t^{-s} \psi_{\lambda}^{*}(t \sqrt{L}) f(x)
$$


This, along with Theorem 3.5, implies

$$
\begin{aligned}
\left\|L^{s / 2} f\right\|_{\dot{B}_{p, q, w}^{\alpha, L}(X)} & \sim\left(\int_{0}^{\infty}\left[t^{-\alpha}\left\|\psi(t \sqrt{L}) L^{s / 2} f\right\|_{p, w}\right]^{q} \frac{d t}{t}\right)^{1 / q} \\
& \lesssim\left(\int_{0}^{\infty}\left[t^{-\alpha-s}\left\|\psi_{\lambda}^{*}(t \sqrt{L}) f\right\|_{p, w}\right]^{q} \frac{d t}{t}\right)^{1 / q} \\
& \sim\|f\|_{\dot{B}_{p, q, w}^{\alpha+s, L}(X)} .
\end{aligned}
$$

Arguing similarly by using the item (b) in Theorem 3.5, we obtain

$$
\left\|L^{s / 2} f\right\|_{\dot{F}_{p, q, w}^{\alpha, L}(X)} \lesssim\|f\|_{\dot{F}_{p, q, w}^{\alpha+s, L}(X)} .
$$

This completes our proof.

7.2. Spectral multiplier of Laplace transform type. Let $m:[0, \infty) \rightarrow \mathbb{C}$ be a bounded function. We now define

$$
\tilde{m}(L)=\int_{0}^{\infty} t L e^{-t^{2} L} m\left(t^{2}\right) d t
$$

to be the spectral multiplier of Laplace transform type of $L$. We have the following result.

THEOREM 7.2. Let $\alpha \in \mathbb{R}$ and $w \in A_{\infty}$. Then the spectral multiplier of Laplace transform type $\tilde{m}(L)$ defined by (102) is bounded on $\dot{B}_{p, q, w}^{\alpha, L}(X)$ for $0<p, q \leqslant \infty$ and is bounded on $\dot{F}_{p, q, w}^{\alpha, L}(X)$ for $0<p<\infty$ and $0<q \leqslant \infty$.

Proof. We will provide the proof for the Triebel-Lizorkin spaces. The boundedness on the Besov spaces can be proved similarly.

Let $\psi$ be a partition of unity. For $f \in \dot{F}_{p, q, w}^{\alpha, L}(X)$, we have

$$
\begin{aligned}
\psi(s \sqrt{L}) \tilde{m}(L) f(x) & =c_{\psi} \int_{s / 4}^{4 s} \psi(u \sqrt{L}) \tilde{m}(L) \psi(s \sqrt{L}) f \frac{d u}{u} \\
& =c_{\psi} \int_{s / 4}^{4 s} \int_{0}^{\infty} m\left(t^{2}\right)\left(t^{2} L\right) e^{-t^{2} L} \psi(u \sqrt{L}) \psi(s \sqrt{L}) f(x) \frac{d t}{t} \frac{d u}{u} \\
& =c_{\psi} \int_{s / 4}^{4 s} \int_{0}^{u} \cdots+c_{\psi} \int_{s / 4}^{4 s} \int_{u}^{+\infty} \cdots \\
& =: E(x)+F(x) .
\end{aligned}
$$


Fix $\lambda>\max \left\{n / q, n q_{w} / p\right\}$ and $N>n$. From Lemmas 2.6 and 2.2, we have

$$
\begin{aligned}
|E(x)| \lesssim \int_{s / 4}^{4 s} \int_{0}^{u} \frac{t^{2}}{u^{2}} \frac{1}{V(y, u)}\left(1+\frac{d(x, y)}{u}\right)^{-N-\lambda}|\psi(s \sqrt{L}) f(y)| d \mu(y) \frac{d t}{t} \frac{d u}{u} \\
\sim \int_{s / 4}^{4 s} \int_{0}^{u} \int_{X} \frac{t^{2}}{u^{2}} \frac{1}{V(y, s)}\left(1+\frac{d(x, y)}{s}\right)^{-N-\lambda}|\psi(s \sqrt{L}) f(y)| d \mu(y) \frac{d t}{t} \frac{d u}{u} \\
\sim \int_{s / 4}^{4 s} \int_{0}^{u} \frac{t^{2}}{u^{2}} \psi_{\lambda}^{*}(s \sqrt{L}) f(x) \frac{d t}{t} \frac{d u}{u} \sim \psi_{\lambda}^{*}(s \sqrt{L}) f(x) .
\end{aligned}
$$

Similarly, for $M>\lambda / 2$,

$$
\begin{aligned}
|F(x)| \lesssim & \int_{s / 4}^{4 s} \int_{u}^{\infty}\left(\frac{u}{t}\right)^{2 M} \frac{1}{V(y, t)}\left(1+\frac{d(x, y)}{t}\right)^{-N-\lambda} \\
& \times|\psi(s \sqrt{L}) f(y)| d \mu(y) \frac{d t}{t} \frac{d u}{u} \\
\sim & \int_{s / 4}^{4 s} \int_{u}^{\infty} \int_{X}\left(\frac{u}{t}\right)^{2 M-\lambda} \frac{1}{V(y, t)}\left(1+\frac{d(x, y)}{t}\right)^{-N}\left(1+\frac{d(x, y)}{u}\right)^{-\lambda} \\
& \times|\psi(s \sqrt{L}) f(y)| d \mu(y) \frac{d t}{t} \frac{d u}{u} \\
\sim & \int_{s / 4}^{4 s} \int_{u}^{\infty}\left(\frac{u}{t}\right)^{2 M-\lambda} \psi_{\lambda}^{*}(s \sqrt{L}) f(x) \frac{d t}{t} \frac{d u}{u} \\
\sim & \psi_{\lambda}^{*}(s \sqrt{L}) f(x) .
\end{aligned}
$$

As a consequence,

$$
|\psi(s \sqrt{L}) \tilde{m}(L) f(x)| \lesssim \psi_{\lambda}^{*}(s \sqrt{L}) f(x) .
$$

Therefore, the conclusion of the theorem follows immediately from Theorem 3.5.

REMARK 7.3. Theorem 7.2 only requires the Gaussian upper bound condition for the operator $L$. This is a very mild condition and allows us to apply the results to a large number of applications such as the sub-Laplacians on Lie groups of polynomial growth, the Laplacians on the Heisenberg groups or the Laplace-Beltrami operators on certain Riemannian manifolds. For further details concerning examples satisfying this condition, we refer to [30, Section 7] and the references therein. It is natural to ask the question on the sharp estimate for the general spectral multipliers of $L$. This problem is more complicated and we leave it as an upcoming project. 


\subsection{Dispersive estimates and Strichartz estimates}

7.3.1. Dispersive estimates and Strichartz estimates for abstract operators. In this section, we assume that $(X, d, \mu)$ further satisfies the following condition:

$$
V(x, r) \gtrsim r^{n} \text { for some } n>1 \text { and for all } x \in X \text { and } r>0 .
$$

Let $L$ be a nonnegative self-adjoint operator on $L^{2}(X)$ satisfying the Gaussian upper bound (GE). In addition, we assume that the operator $L$ satisfies the following assumptions:

(D) There exists $T>0$ so that $L$ satisfies the standard $L^{1}-L^{\infty}$ dispersive estimates for all $|t|<T$

$$
\left|e^{i t L} f\right| \leqslant|t|^{-n / 2}\|f\|_{1} .
$$

The dispersive estimate (D) plays an essential role in obtaining the Strichartz estimate. See, for example, [48]. We now discuss some classes of operators which satisfy conditions (D):

(a) It is worth noticing that the Laplacian on $\mathbb{R}^{n}$ satisfies (D) with $T=\infty$. Moreover, the Hermite operators $L=-\Delta+|x|^{2}$ on $\mathbb{R}^{n}$, the twisted Laplacian on $\mathbb{R}^{2 n}$

$$
L=\frac{1}{2} \sum_{j=1}^{n}\left[\left(\partial_{x_{j}}-i y_{j}\right)^{2}+\left(\partial_{y_{j}}-i x_{j}\right)^{2}\right]
$$

and the second-order differential operators on Heisenberg groups considered in [53] also satisfy the condition (D) with any $T<\pi / 2$. See, for example, [29, 53].

(b) Let $L=-\Delta+V$ be a Schrödinger operator in $\mathbb{R}^{3}$ with the potential $V$ satisfying the following conditions:

$$
\int_{\mathbb{R}^{3} \times \mathbb{R}^{3}} \frac{|V(x)||V(y)|}{|x-y|^{2}} d x d y<(4 \pi)^{2}
$$

and

$$
\|V\|_{\mathcal{K}}=: \sup _{x \in \mathbb{R}^{3}} \int_{\mathbb{R}^{3}} \frac{|V(y)|}{|x-y|} d y<4 \pi .
$$

Then, $L$ satisfies (D). See [56, Theorem 2.3].

(c) Consider the following Schrödinger operator $L=-\Delta+V\left(x_{1}\right)+\cdots+V\left(x_{n}\right)$ on $\mathbb{R}^{n}$. Assume that the potential $V \geqslant 0$ is a real-valued function satisfying $\left(1+|x|^{2}\right) V(x) \in L^{1}(\mathbb{R})$. Then $L$ satisfies (D) for $T=\infty$. See, for example, [55, Corollary 1.6]. 
(d) Let $L=-\Delta+V$ be a Schrödinger operator on $\mathbb{R}^{n}$ with $V \geqslant 0$. We state a set of sufficient conditions for the operator $L$ to satisfy (D) for $T=\infty$ (see [65]): $n \geqslant 3, p_{0}>n / 2, \delta>3 n / 2+1, \ell_{0}=0$ if $n=3$ and $\ell_{0}=[(n-1) / 2]$ if $n \geqslant 4$, and $V: \mathbb{R}^{n} \rightarrow \mathbb{R}$ satisfies

$$
\left\|\partial^{\alpha} V\right\|_{L^{p_{0}(|x-y| \leqslant 1)}} \leqslant \frac{C}{(1+|x|)^{\delta}}, \quad \forall|\alpha| \leqslant \ell_{0} .
$$

(e) Apart from the above examples, the dispersive estimate (D) is satisfied for the wide range of Schrödinger operators on product manifolds and for Laguerre operators. For further details, we refer the reader to $[\mathbf{1 5}, \mathbf{5 5}]$.

Our main aim in this section is to study the dispersive estimates related to the flow $e^{i t \sqrt{L}}$. It is well known that $e^{i t \sqrt{L}}$ can describe the solutions to the wave equation

$$
u_{t t}+L u=0
$$

via the following identities

$$
\cos (t \sqrt{L})=\frac{e^{i t \sqrt{L}}+e^{-i t \sqrt{L}}}{2}, \quad \sin (t \sqrt{L})=\frac{e^{i t \sqrt{L}}-e^{-i t \sqrt{L}}}{2 i} .
$$

We recall the following estimate in [15].

THEOREM 7.4. Assume that $(X, d, \mu)$ satisfies (103). Let $\psi \in C_{c}^{\infty}\left(\left[\frac{1}{2}, 2\right]\right)$. Then

$$
\left\|e^{i t \sqrt{L}} \psi_{j}(\sqrt{L}) f\right\|_{L^{\infty}} \lesssim 2^{j \frac{n+1}{2}}|t|^{-\frac{n-1}{2}}\|f\|_{L^{1}},|t|<T, f \in L^{2},
$$

where $\psi_{j}=\psi\left(2^{-j} \cdot\right)$ for all $j \in \mathbb{Z}$.

THEOREM 7.5. Assume that $(X, d, \mu)$ satisfies (103) and L satisfies the Gaussian upper bound (GE) and (D). Then for $|t|<T$, we have

$$
\begin{gathered}
\left\|e^{i t \sqrt{L}} f\right\|_{\dot{B}_{\infty, 2}^{-\frac{n+1}{4}, L}} \lesssim|t|^{-\frac{n-1}{2}}\|f\|_{\dot{B}_{1,2}^{\frac{n+1}{4}, L}}, \\
\left\|e^{i t \sqrt{L}} f\right\|_{L^{\infty}} \lesssim|t|^{-\frac{n-1}{2}}\|f\|_{\dot{B}_{1,1}^{\frac{n+1}{2}, L}}
\end{gathered}
$$

and

$$
\left\|\frac{e^{i t \sqrt{L}}}{\sqrt{L}} f\right\|_{L^{\infty}} \lesssim|t|^{-\frac{n-1}{2}}\|f\|_{\dot{B}_{1,1}^{\frac{n-1}{2}, L}} .
$$


Proof. We give the proof for (107) only, as the other two estimates can be verified in the same manner. Let $\psi \in C_{c}^{\infty}([1 / 2,2])$ be a partition of unity, that is,

$$
\sum_{j \in \mathbb{Z}} \psi_{j}(\lambda)=1, \forall \lambda>0
$$

Using the fact that $\psi_{j}=\psi_{j}\left(\psi_{j+1}+\psi_{j}+\psi_{j-1}\right)$ for each $j \in \mathbb{Z}$ and Theorem 7.4, we have, for each $v \in \mathbb{Z}$,

$$
\begin{aligned}
& \left\|\psi_{\nu}(\sqrt{L}) e^{i t \sqrt{L}} f\right\|_{L^{\infty}} \\
& \quad \leqslant\left\|\left(\psi_{\nu+1}(\sqrt{L})+\psi_{\nu}(\sqrt{L})+\psi_{\nu-1}(\sqrt{L})\right) \psi_{\nu}(\sqrt{L}) e^{i t \sqrt{L}} f\right\|_{L^{\infty}} \\
& \quad \lesssim 2^{v \frac{n+1}{2}}|t|^{-\frac{n-1}{2}}\left\|\psi_{\nu}(\sqrt{L}) f\right\|_{L^{1}} .
\end{aligned}
$$

It follows

$$
2^{v \frac{n+1}{4}}\left\|\psi_{\nu}(\sqrt{L}) e^{i t \sqrt{L}} f\right\|_{L^{\infty}} \lesssim|t|^{-\frac{n-1}{2}} 2^{-v \frac{n+1}{4}}\left\|\psi_{\nu}(\sqrt{L})(L) e^{i t \sqrt{L}} f\right\|_{L^{1}}
$$

which proves (107).

The estimates in Theorem 7.5 lead to the following results concerning the propagator $e^{i t \sqrt{L}}$.

THEOREM 7.6. Assume that $(X, d, \mu)$ satisfies (103) and L satisfies the Gaussian upper bound (GE) and (D). Then for $2 \leqslant q<\infty$, we have

$$
\left\|e^{i t \sqrt{L}} f\right\|_{L^{q}} \lesssim|t|^{-\frac{n-1}{2}\left(1-\frac{2}{q}\right)}\|f\|_{\dot{B}_{q^{\prime}, q}^{\frac{n+1}{2}\left(1-\frac{2}{q}\right), L}}
$$

and

$$
\left\|\frac{e^{i t \sqrt{L}}}{\sqrt{L}} f\right\|_{L^{q}} \lesssim|t|^{-\frac{n-1}{2}\left(1-\frac{2}{q}\right)}\|f\|_{\dot{B}_{q^{\prime}, q}^{-\frac{1}{q}+\frac{n-1}{2}\left(1-\frac{2}{q}\right), L}}
$$

for all $|t|<T$.

Proof. We first note that

$$
\left\|e^{i t \sqrt{L}} f\right\|_{L^{2}} \leqslant\|f\|_{L^{2}} .
$$

Since $L^{2}(X) \equiv \dot{B}_{2,2}^{0, L}(X)$ (see Theorem 5.1), this implies that

$$
\|f\|_{L^{2}} \approx\|f\|_{\dot{B}_{2,2}^{0, L}}
$$

This together with (108) implies that

$$
\left\|e^{i t \sqrt{L}} f\right\|_{\left(L^{2}, L^{\infty}\right)_{(\theta, q)}} \lesssim|t|^{-\frac{n-1}{2} \theta}\|f\|_{\left(\dot{B}_{2,2}^{0, L}, \dot{B}_{1,1}^{\frac{n+1}{2}, L}\right)_{\theta, q}} .
$$


Taking $\theta=1-2 / q$ and using the interpolation theorem in [18, Proposition 3.18], we get (110).

Similarly, from Theorem 5.7, we have

$$
\left\|L^{-1 / 2} f\right\|_{L^{2}} \approx\|f\|_{\dot{B}_{2,2}^{-1 / 2, L}}
$$

which implies

$$
\left\|\frac{e^{i t \sqrt{L}}}{\sqrt{L}} f\right\|_{L^{2}} \lesssim\|f\|_{\dot{B}_{2,2}^{-1 / 2, L}} .
$$

At this stage, using the real interpolation as above, we get (111).

Another implication of Theorem 7.5 is the Strichartz estimates for the flow $e^{i t \sqrt{L}}$ on the new Besov spaces scale.

Definition 7.7. For $\sigma>0$. We say that the pair of exponents $(q, \theta)$ is sharp $\sigma$-admissible if $(q, \theta, \sigma) \neq(2,1,1), 2 \leqslant q=\frac{2}{\sigma \theta}, \theta \in[0,1]$.

We say that the pair of exponents $(q, \theta)$ is $\sigma$-acceptable if either $q \in[1, \infty)$, $\theta \in[0,1]$ and $q>\frac{1}{\sigma \theta}$ or $(q, \theta)=(\infty, 0)$.

THEOREM 7.8. Assume that $(X, d, \mu)$ satisfies (103) and L satisfies the Gaussian upper bound $(\mathrm{GE})$ and $(D)$ with $T=\infty$. Then for all sharp $\frac{n-1}{2}$-admissible pairs $(q, \theta)$ and $(\tilde{q}, \tilde{\theta})$, the following Strichartz estimates hold true:

$$
\begin{gathered}
\left\|e^{i t \sqrt{L}} f\right\|_{L_{t}^{q} B_{\theta}^{\prime}} \lesssim\|f\|_{L^{2}}, \\
\left\|\int_{0}^{\infty} e^{-i s \sqrt{L}} F(s) d s\right\|_{L^{2}} \lesssim\|F\|_{L_{t}^{q^{\prime}} B_{\theta}}
\end{gathered}
$$

and

$$
\left\|\int_{s<t} e^{i(t-s) \sqrt{L}} F(s) d s\right\|_{L_{t}^{q} B_{\theta}^{\prime}} \lesssim\|F\|_{L_{t}^{\tilde{q}^{\prime}} B_{\tilde{\theta}}},
$$

where $B_{\theta}=\left(\dot{B}_{2,2}^{0, L}, \dot{B}_{1,2}^{\frac{n+1}{4}, L}\right)_{\theta, 2}$.

Proof. We first note that by Theorem 5.1, we have

$$
\left\|e^{i t \sqrt{L}} f\right\|_{L^{2}} \leqslant\|f\|_{L^{2}} \approx\|f\|_{\dot{B}_{2,2}^{0, L}} .
$$

Note that from the atomic decomposition theorem and the standard argument (see, for example, [42, Theorem 8.11]), we can show that

$$
\dot{B}_{\infty, 2}^{-\frac{n+1}{4}, L}=\left(\dot{B}_{1,2}^{\frac{n+1}{4}, L}\right)^{\prime}
$$


This and (107) imply

$$
\left\|e^{i t \sqrt{L}} f\right\|_{\left(\dot{B}_{1,2}^{\frac{n+1}{4}, L}\right)^{\prime}} \lesssim|t|^{-\frac{n-1}{2}}\|f\|_{\dot{B}_{1,2}^{\frac{n+1}{4}, L}} .
$$

Hence, the statements of the theorem follow immediately by [48, Theorem 10.1].

REMARK 7.9. The inhomogeneous estimate (116) can be improved for all $\frac{n-1}{2}-$ acceptable pairs as in [59, Theorem 1.4]. We do not pursue this problem and leave it to the interested reader.

7.3.2. Dispersive estimates for Schrödinger operators with large rough potentials. As in [28], we consider the following Schrödinger operator $L=-\Delta+V$ on $\mathbb{R}^{n}, n \geqslant 3$. For a potential $V$, we say that $V$ belongs to the Kato class if

$$
\lim _{r \rightarrow 0} \sup _{x \in \mathbb{R}^{n}} \int_{|x-y|<r} \frac{|V(y)|}{|x-y|^{n-2}} d y=0 .
$$

Moreover, the Kato norm of $V$ is defined by

$$
\|V\|_{\mathcal{K}}=\sup _{x \in \mathbb{R}^{n}, r>0} \int_{|x-y|<r} \frac{|V(y)|}{|x-y|^{n-2}} d y .
$$

Note that all results in this section hold true for $n \geqslant 3$. However, for the sake of simplicity, we consider the particular case $n=3$.

As in [28], we assume the following conditions:

(a) $V$ is a real potential which belongs to the Kato class;

(b) $V=V_{1}+V_{2}$ satisfies

(i) $V_{1}$ is compactly supported and has a bounded Kato norm, and

(ii) $\left\|V_{2}\right\|_{\mathcal{K}}\left(1+\left\|V_{1}\right\|_{\mathcal{K}} / 4 \pi\right)<4 \pi$;

(c) $\left\|V_{-}\right\|_{\mathcal{K}}<2 \pi$ where $V_{-}=\min \{V, 0\}$;

(d) the integral equation $f+R_{0}(\lambda+i 0) V f=0$ has no nontrivial bounded solution for any $\lambda \geqslant 0$, where $R_{0}(z)=(-z-\Delta)^{-1}, z \in \mathbb{C}$.

Under conditions (a)-(d), $L$ is a nonnegative self-adjoint operator satisfying Gaussian upper bound, that is, the kernel $p_{t}(x, y)$ of $e^{-t L}$ satisfies

$$
p_{t}(x, y) \leqslant \frac{C}{t^{n / 2}} \exp \left(-\frac{|x-y|^{2}}{c t}\right) .
$$

We now prove the following estimates. 
THEOREM 7.10. Assume that L satisfies $(a)-(d)$. Then for all $t>0$, we have

$$
\begin{aligned}
\left\|e^{i t \sqrt{L}} f\right\|_{\dot{B}_{\infty, 2}^{-1, L}} & \lesssim t^{-1}\|f\|_{\dot{B}_{1,2}^{1, L}}, \\
\left\|e^{i t \sqrt{L}} f\right\|_{L^{\infty}} & \lesssim t^{-1}\|f\|_{\dot{B}_{1,1}^{2, L}}
\end{aligned}
$$

and

$$
\left\|\frac{e^{i t \sqrt{L}}}{\sqrt{L}} f\right\|_{L^{\infty}} \lesssim t^{-1}\|f\|_{\dot{B}_{1,1}^{1, L}} .
$$

Proof. Similarly to Theorem 7.5, it suffices to prove that for $\psi \in C_{c}^{\infty}\left(\left[\frac{1}{2}, 2\right]\right)$,

$$
\left\|e^{i t \sqrt{L}} \psi_{j}(\sqrt{L}) f\right\|_{L^{\infty}} \lesssim 2^{2 j} t^{-1}\|f\|_{L^{1}}, \quad t>0
$$

where $\psi_{j}=\psi\left(2^{-j} \cdot\right)$ for all $j \in \mathbb{Z}$.

Indeed, by an argument in [28, pages 74-76], we obtain that

$$
\left\|\sin (t \sqrt{L}) \psi_{j}(\sqrt{L}) f\right\|_{L^{\infty}} \lesssim 2^{2 j} t^{-1}\|f\|_{L^{1}}
$$

and

$$
\left\|\cos (t \sqrt{L}) \psi_{j}(\sqrt{L}) f\right\|_{L^{\infty}} \lesssim 2^{2 j} t^{-1}\|f\|_{L^{1}} .
$$

Hence, this proves (120).

Similarly to Theorem 7.8 , we also obtain the Strichartz estimate for the flow $e^{i t \sqrt{L}}$.

THEOREM 7.11. Assume that L satisfies $(a)-(d)$. Then for all sharp 1-admissible pairs $(q, \theta)$ and $(\tilde{q}, \tilde{\theta})$, the following Strichartz estimates hold true:

$$
\begin{gathered}
\left\|e^{i t \sqrt{L}} f\right\|_{L_{t}^{q} B_{\theta}^{\prime}} \lesssim\|f\|_{L^{2}}, \\
\left\|\int_{0}^{\infty} e^{-i s \sqrt{L}} F(s) d s\right\|_{L^{2}} \lesssim\|F\|_{L_{t}^{q^{\prime}} B_{\theta}}
\end{gathered}
$$

and

$$
\left\|\int_{s<t} e^{i(t-s) \sqrt{L}} F(s) d s\right\|_{L_{t}^{q} B_{\theta}^{\prime}} \lesssim\|F\|_{L_{t}^{\tilde{q}^{\prime}} B_{\tilde{\theta}}},
$$

where $B_{\theta}=\left(\dot{B}_{2,2}^{0, L}, \dot{B}_{1,2}^{1, L}\right)_{\theta, 2}$.

\section{Acknowledgements}

Xuan Thinh Duong was supported by Australian Research Council through the ARC grant DP160100153 and Macquarie University Research grant 82184614 . The authors would like to thank the referees for their useful comments and suggestions which have helped to improve the paper. 


\section{Conflict of Interest: None.}

\section{References}

[1] N. Aguilera and C. Segovia, 'Weighted norm inequalities relating the $g_{\lambda}^{*}$ and the area functions', Studia Math. 61(3) (1977), 293-303.

[2] P. Auscher, X. T. Duong and A. McIntosh, 'Boundedness of Banach space valued singular integral operators and Hardy spaces', Preprint, 2005 (unpublished).

[3] P. Auscher and J. M. Martell, 'Weighted norm inequalities, off-diagonal estimates and elliptic operators. Part III: harmonic Analysis of elliptic operators', J. Funct. Anal. 241 (2006), 703-746.

[4] O. V. Besov, 'On a family of function spaces, embedding theorems and extensions', Dokl. Akad. Nauk SSSR 126 (1959), 1163-1165 (in Russian).

[5] O. V. Besov, 'On a family of function spaces in connection with embeddings and extensions', Tr. Mat. Inst. Steklova 60 (1961), 42-81 (in Russian).

[6] O. V. Besov, V. P. II'in and S. M. Nikol'skii, Integral Representation of Functions and Embedding Theorems (ed. V. H. Winston) vol. I (Sons, Washington, DC, 1979), vol. II.

[7] H.-Q. Bui, T. A. Bui and X. T. Duong, 'New test distribution spaces, Besov and TriebelLizorkin spaces associated to operators and applications to dispersive estimates', Preprint (unpublished).

[8] H.-Q. Bui and T. Candy, 'A characterisation of the Besov-Lipschitz and Triebel-Lizorkin spaces using Poisson like kernels', Contemp. Maths 693 (2017), 109-141.

[9] H.-Q. Bui, X. T. Duong and L. X. Yan, 'Calderón reproducing formulas and new Besov spaces associated with operators', Adv. Math. 229 (2012), 2449-2502.

[10] H.-Q. Bui, M. Paluszýnski and M. H. Taibleson, 'A note on the Besov-Lipschitz and TriebelLizorkin spaces’, Contemp. Math. 189 (1995), 95-101.

[11] H.-Q. Bui, M. Paluszýnski and M. H. Taibleson, 'A maximal function characterization of weighted Besov-Lipschitz and Triebel-Lizorkin spaces', Studia Math. 119 (1996), 219-246.

[12] H.-Q. Bui, M. Paluszýnski and M. H. Taibleson, 'Characterization of the Besov-Lipschitz and Triebel-Lizorkin spaces. The case $q<1$ ', J. Fourier Anal. Appl. 3 (1997), 837-846.

[13] H.-Q. Bui and M. H. Taibleson, 'The characterization of the Triebel-Lizorkin spaces for $p=\infty$, J. Fourier Anal. Appl. 6 (2000), 537-550.

[14] T. A. Bui, J. Cao, L. D. Ky, D. Yang and S. Yang, 'Musielak-Orlicz-Hardy spaces associated with operators satisfying reinforced off-diagonal estimates', Anal. Geom. Metr. Spaces 1 (2013), 69-129.

[15] T. A. Bui, P. D'Ancona, X. T. Duong and D. Müller, 'On the flows associated to selfadjoint operators on metric measure spaces', Math. Ann. 375 (2019), 1393-1426.

[16] T. A. Bui and X. T. Duong, 'Besov and Triebel-Lizorkin spaces associated to Hermite operators', J. Fourier Anal. Appl. 21(2) (2015), 405-448.

[17] T. A. Bui and X. T. Duong, 'Laguerre operator and its associated weighted Besov and TriebelLizorkin spaces', Trans. Amer. Math. Soc. 369(3) (2017), 2109-2150.

[18] T. A. Bui and X. T. Duong, 'Spectral multipliers of self-adjoint operators on Besov and Triebel-Lizorkin spaces associated to operators', Int. Math. Res. Not. IMRN (to appear).

[19] T. A. Bui and X. T. Duong, 'Weighted BMO spaces associated to operators', Proceedings of the AMSI/AustMS 2014 Workshop in Harmonic Analysis and its Applications, 725, Proc. Centre Math. Appl. Austral. Nat. Univ., 47 (Center of Mathematics Analysis at ANU, 2017). 
[20] T. A. Bui, X. T. Duong and Y. Hong, 'Dispersive and Strichartz estimates for the threedimensional wave equation with a scaling-critical class of potentials', J. Funct. Anal. 271 (2016), 2215-2246.

[21] T. A. Bui, X. T. Duong and F. K. Ly, 'Maximal function characterizations for new local Hardy type spaces on spaces of homogeneous type', Trans. Amer. Math. Soc. 370(10) (2018), 7229-7292.

[22] A. P. Calderón and A. Torchinsky, 'Parabolic maximal functions associated with a distribution', Adv. Math. 16 (1975), 1-64.

[23] M. Christ, 'A $T(b)$ theorem with remarks on analytic capacity and the Cauchy integral', Colloq. Math. 61 (1990), 601-628.

[24] R. R. Coifman and G. Weiss, Analyse Harmonique Non-commutative sur Certains Espaces Homogenes, Lecture Notes in Mathematics, 242 (Springer, Berlin-New York, 1971).

[25] T. Coulhon and X. T. Duong, 'Maximal regularity and kernel bounds: observations on a theorem by Hieber and Prüss', Adv. Differential Equ. 5 (2000), 343-368.

[26] T. Coulhon, G. Kerkyacharian and P. Petrushev, 'Heat kernel generated frames in the setting of Dirichlet spaces', J. Fourier Anal. Appl. 18(5) (2012), 995-1066.

[27] T. Coulhon and A. Sikora, 'Gaussian heat kernel upper bounds via Phragmén-Lindelöf theorem', Proc. Lond. Math. Soc. (3) 96 (2008), 507-544.

[28] P. D'Ancona and V. Pierfelice, 'On the wave equation with a large rough potential', J. Funct. Anal. 227(1) (2005), 30-77.

[29] P. D'Ancona, V. Pierfelice and F. Ricci, 'On the wave equation associated to the Hermite and the twisted Laplacian', J. Fourier Anal. Appl. 16 (2010), 294-310.

[30] X. T. Duong, E. M. Ouhabaz and A. Sikora, 'Plancherel-type estimates and sharp spectral multipliers', J. Funct. Anal. 196 (2002), 443-485.

[31] X. T. Duong and L. X. Yan, 'New function spaces of BMO type the John-Nirenberg inequality, interpolation and applications', Comm. Pure Appl. Math. 58 (2005), 1375-1420.

[32] X. T. Duong and L. X. Yan, 'Duality of Hardy and BMO spaces associated with operators with heat kernel bounds', J. Amer. Math. Soc. 18 (2005), 943-973.

[33] X. T. Duong, J. Li and L. X. Yan, 'A Littlewood-Paley type decomposition and weighted Hardy spaces associated with operators', J. Geom. Anal. 26(2) (2016), 1617-1646.

[34] M. Frazier and B. Jawerth, 'Decomposition of Besov spaces', Indiana Univ. Math. J. 34 (1985), 777-799.

[35] M. Frazier and B. Jawerth, 'A discrete transform and decomposition of distribution spaces', J. Funct. Anal. 93 (1990), 34-170.

[36] A. G. Georgiadis, G. Kerkyacharian, G. Kyriazis and P. Petrushev, 'Homogeneous Besov and Triebel-Lizorkin spaces associated to non-negative self-adjoint operators', J. Math. Anal. Appl. 449(2) (2017), 1382-1412.

[37] A. G. Georgiadis, G. Kerkyacharian, G. Kyriazis and P. Petrushev, 'Atomic and molecular decomposition of homogeneous spaces of distributions associated to non-negative self-adjoint operators', J. Fourier Anal. Appl. 25 (2019), 3259-3309.

[38] L. Grafakos, L. Liu and D. Yang, 'Vector-valued singular integrals and maximal functions on spaces of homogeneous type', Math. Scand. 104 (2009), 296-310.

[39] Y. S. Han and E. T. Sawyer, 'Littlewood-Paley theory on spaces of homogeneous type and the classical function spaces', Mem. Amer. Math. Soc. 110(530) (1994), vi+126 pp.

[40] S. X. Hou, D. Yang and S. Yang, 'Musielak-Orlicz BMO-type spaces associated with generalized approximations to the identity', Acta Math. Sin. (Engl. Ser.) 30(11) (2014), 1917-1962. 
[41] G. Hu, 'Littlewood-Paley characterization of weighted Hardy spaces associated with operators', J. Aust. Math. Soc. 103 (2017), 250-267.

[42] Y. S. Han, D. Müller and D. Yang, 'A theory of Besov and Triebel-Lizorkin spaces on metric measure spaces modeled on Carnot-Caratheodory spaces', Abstr. Appl. Anal. (2008), Art. ID $893409,250 \mathrm{pp}$.

[43] N. J. H. Heideman, 'Duality and fractional integration in Lipschitz spaces', Studia Math. 50 (1974), 65-85.

[44] S. Hofmann, G. Lu, D. Mitrea, M. Mitrea and L. Yan, 'Hardy spaces associated to nonnegative self-adjoint operators satisfying Davies-Gaffney estimates', Mem. Amer. Math. Soc. 214(1007) (2011).

[45] S. Hofmann and V. Mayboroda, 'Hardy and BMO spaces associated to divergence form elliptic operators', Math. Ann. 344 (2009), 37-116.

[46] S. Hofmann, V. Mayboroda and A. McIntosh, 'Second order elliptic operators with complex bounded measurable coefficients in $L^{p}$, Sobolev and Hardy spaces', Ann. Sci. Éc. Norm. Supér. (4) 44 (2011), 723-800.

[47] R. Jiang and D. Yang, 'Orlicz-Hardy spaces associated with operators satisfying DaviesGaffney estimates', Commun. Contemp. Math. 13(2) (2011), 331-373.

[48] M. Keel and T. T. Tao, 'End point Strichartz estimates', Amer. J. Math. 120 (1998), 955-980.

[49] C. E. Kenig, G. Ponce and L. Vega, 'Oscillatory integrals and regularity of dispersive equations', Indiana Math. J. 40 (1991), 33-69.

[50] G. Keryacharian and P. Petrushev, 'Heat kernel based decomposition of spaces of distributions in the framework of Dirichlet spaces', Trans. Amer. Math. Soc. 367(1) (2015), 121-189.

[51] G. Kerkyacharian, P. Petrushev, D. Picard and Y. Xu, 'Decomposition of Triebel-Lizorkin and Besov spaces in the context of Laguerre expansions', J. Funct. Anal. 256 (2009), 1137-1188.

[52] L. Liu, D. Yang and W. Yuan, 'Besov-type and Triebel-Lizorkin-type spaces associated with heat kernels', Collect. Math. 67 (2016), 247-310.

[53] D. Müller and F. Ricci, 'Analysis of second order differential operators on Heisenberg groups. I', Invent. Math. 101 (1990), 545-582.

[54] P. Petrushev and Y. Xu, 'Decomposition of spaces of distributions induced by Hermite expansions', J. Fourier Anal. Appl. 14(3) (2008), 372-414.

[55] J. Peetre, New Thoughts on Besov Spaces, Duke University Mathematica Series (Durham, NC, 1976).

[56] W. Schlag, 'Dispersive estimates for Schrödinger operators: a survey', in Mathematical Aspects of Nonlinear Dispersive Equations, 255285, Annals of Mathematical Studies, 163 (Princeton University Press, Princeton, NJ, 2007).

[57] A. Sikora, 'Riesz transform, Gaussian bounds and the method of wave function', Math. Z. 247 (2004), 643-662.

[58] J.-O. Strömberg and A. Torchinsky, Weighted Hardy Spaces (Springer, Berlin, 1989).

[59] R. J. Taggart, 'Inhomogeneous Strichartz estimates', Forum Math. 22(5) (2010), 825-853.

[60] H. Triebel, Theory of Function Spaces, Monographs in Mathematics, 78 (Birkhäuser, Basel, 1983).

[61] H. Triebel, Theory of Function Spaces II, Monographs in Mathematics, 84 (Birkhäuser, Basel, 1992).

[62] S. Yang and D. Yang, 'Atomic and maximal function characterizations of Musielak-OrliczHardy spaces associated to non-negative self-adjoint operators on spaces of homogeneous type', Collect. Math. 70(2) (2019), 197-246.

[63] D. Yang and W. Yuan, 'Pointwise characterizations of Besov and Triebel-Lizorkin spaces in terms of averages on balls', Trans. Amer. Math. Soc. 369 (2017), 7631-7655. 
[64] D. Yang and S. Yang, 'Musielak-Orlicz-Hardy spaces associated with operators and their applications', J. Geom. Anal. 24(1) (2014), 495-570.

[65] K. Yajima, 'The $W^{k, p}$-continuity of wave operators for Schrödinger operators. III. Evendimensional cases $m \geqslant 4$ ', J. Math. Sci. Univ. Tokyo 2 (1995), 311-346.

[66] W. Yuan, W. Sickel and D. Yang, Morrey and Campanato Meet Besov, Lizorkin and Triebel, Lecture Notes in Mathematics, 2005 (Springer, Berlin, 2010). 